MARLON GRIPP CHERMONT

PROPOSTA DE DESENVOLVIMENTO DE UM AGENTE PROXY SNMP PARA GERENCIAMENTO DE REDES LONWORKS 
MARLON GRIPP CHERMONT

\section{PROPOSTA DE DESENVOLVIMENTO DE UM AGENTE PROXY SNMP PARA GERENCIAMENTO DE REDES LONWORKS}

Dissertação apresentada à Escola Politécnica da Universidade de São Paulo para obtenção do Título de Mestre em Engenharia.

Área de Concentração:

Sistemas Digitais

Orientador: Prof. Livre Docente Carlos Eduardo Cugnasca 


\section{FICHA CATALOGRÁFICA}

\section{Chermont, Marlon Gripp}

Proposta de desenvolvimento de um agente Proxy SNMP para gerenciamento de redes LonWorks / M.G. Chermont. -- São Paulo, 2007.

$$
\text { p. } 83
$$

Dissertação (Mestrado) - Escola Politécnica da Universidade de São Paulo. Departamento de Engenharia de Computação e Sistemas Digitais.

1.Agente 2.Proxy 3.SNMP 4.LonWorks 5.Gerenciamento de redes 6.Automação predial I.Universidade de São Paulo. Escola Politécnica. Departamento de Engenharia de Computação e Sistemas Digitais II.t. 


\section{DEDICATÓRIA}

Aos meus pais e meus irmãos que torcem por mim, mesmo eu não estando tão próximo deles como eu gostaria, e aos meus amigos que sempre estão ao meu lado me incentivando. 


\section{AGRADECIMENTOS}

A todos os meus amigos que estão sempre ao meu lado me incentivando.

A toda equipe da P2S Tecnologia que me apoiou e me incentivou na conclusão deste trabalho, principalmente o meu sócio Sergio Roberto de Mello Canovas que esteve presente em cada momento da execução deste trabalho.

Agradeço também ao meu orientador Carlos Eduardo Cugnasca pela paciência e por acreditar que esse trabalho poderia ser realizado.

Ao professor Miguel dos Santos Alves Filho pela orientação nos estudos da tecnologia LonWorks.

Ao pesquisador Rafael Fernandes Lopes que forneceu a API capaz de agilizar o desenvolvimento desse trabalho.

Às empresas TAC, Schneider Electric, PureChoice e Conceito Tecnologia pelo fornecimento de módulos e componentes utilizados para o desenvolvimento deste trabalho.

À Fundação de Amparo à Pesquisa do Estado de São Paulo (FAPESP), que através do apoio ao projeto TIDIA permitiu a aquisição de equipamentos utilizados neste trabalho. 


\section{RESUMO}

CHERMONT, M. G. Proposta de Desenvolvimento de um Agente Proxy SNMP para Gerenciamento de Redes LonWorks. 2007. 83 p. Dissertação (Mestrado) Escola Politécnica, Universidade de São Paulo, São Paulo, 2007.

Com o crescimento da utilização das redes de computadores e também o aumento da utilização das redes de dispositivos para a automação, torna-se cada vez mais atrativo se pensar em uma solução que integre essas tecnologias. Em cada uma das áreas se observa um aumento da utilização da tecnologia LonWorks em redes distribuídas de dispositivos, e a consolidação do Simple Network Management Protocol (SNMP) como um protocolo padrão para o gerenciamento das redes de computadores. Neste trabalho foi desenvolvido um agente proxy SNMP, capaz de auxiliar no gerenciamento de uma rede de automação baseada na tecnologia LonWorks através do uso de ferramentas já disponíveis no mercado para a realização do gerenciamento das redes de computadores. Esse tipo de solução possibilita que uma mesma equipe possa gerenciar as duas estruturas de rede, através de um mesmo sistema, facilitando a interação entre os dispositivos e reduzindo o custo atrelado à manutenção das equipes. $O$ agente foi desenvolvido através da utilização de uma Application Programming Interface (API), que implementa toda a estrutura de comunicação SNMP; e para realizar o gerenciamento foi criada uma Management Information Base (MIB), com algumas informações de diagnóstico do dispositivo.

Palavras-chave: Agente. Proxy. SNMP. LonWorks. Gerenciamento de redes. Automação predial. 


\begin{abstract}
CHERMONT, M.G. Proposal for Development an Agent Proxy SNMP for LonWorks Network Management. 2007. 83 p. Master thesis - Escola Politécnica, Universidade de São Paulo, São Paulo, 2007.

With the growth of the computer networks use and also the increase of devices network for the automation use, becomes each more attractive think about a solution that integrates these technologies. In each one of those areas it observes, an increase of the LonWorks technology use in distributed networks of devices, and the consolidation of the Simple Network Management Protocol (SNMP) as a protocol standard for the management of the computer networks. In this work was developed a proxy agent SNMP, capable to assist the management of control network, based on the LonWorks technology, through the available tools use already in the market for the accomplishment of the computer networks management. This solution type makes possible that same team can manage two network structures, through the same system, facilitating the interaction between the devices and reducing maintenance teams cost. The agent was developed through of an Application Programming Interface (API) use, that implements all the communication structure SNMP; and to achieve the management was created a Management Information Base (MIB), with some information of devices diagnosis.
\end{abstract}

Keywords: Agent. Proxy. SNMP. LonWorks. Management network. Building Automation 


\section{LISTA DE FIGURAS}

Figura 1. Estrutura de nomenclatura de objetos no SNMP ...............................19

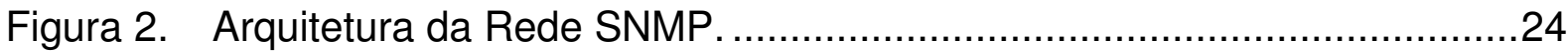

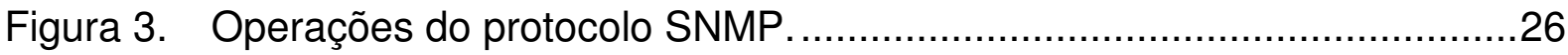

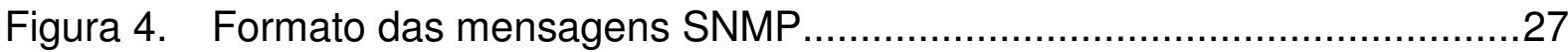

Figura 5. Agente SNMP implementado em cada dispositivo da rede de controle. .31

Figura 6. Arquitetura do Agente Proxy SNMP............................................... 32

Figura 7. Tendência das soluções de automação.........................................33

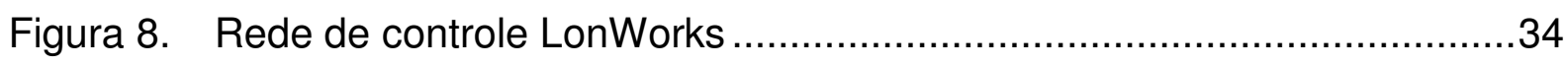

Figura 9. Elementos chaves da tecnologia LonWorks.....................................35

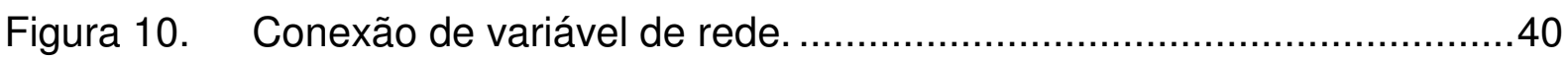

Figura 11. Dispositivo com diferentes perfis funcionais. .................................42

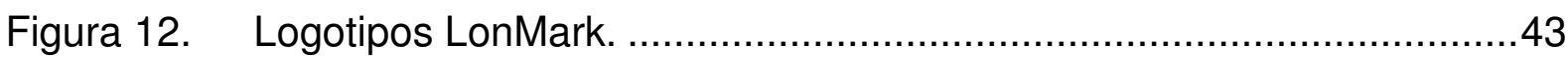

Figura 13. Dispositivo LonWorks. ...................................................... 44

Figura 14. Arquitetura da solução proposta............................................48

Figura 15. Objeto NodeTable de informações dos dispositivos. .........................51

Figura 16. Objeto NodeDiagTable de informações de diagnóstico do dispositivo.52

Figura 17. Classe Node criada para representar um dispositivo........................53

Figura 18. Estrutura interna do agente proxy SNMP. ......................................55

Figura 19. Teste de requisição do objeto MIB SysContact implementado no agente.....

Figura 20. Teste de requisição do status do dispositivo através da classe gateway.. .58

Figura 21. Requisição do status do dispositivo através do programa NodeUtil. ...59

Figura 22. Tela do programa MibBrowser consultando as informações do agente proxy SNMP .60

Figura 23. Tempo médio de requisição e atualização em uma rede com dois nós e taxa de varredura de $2.000 \mathrm{~ms}$.

Figura 24. Porcentagem de falha na mensagem de requisição de atualização do dispositivo LonWorks. .62

Figura 25. Tempo médio de atualização em uma rede com 64 nós e taxa de varredura de $7.040 \mathrm{~ms}$. 
Figura 26. Porcentagem de sucesso na mensagem de requisição de atualização do dispositivo LonWorks com melhorias no agente. ........................................64

Figura 27. Processo de implementação de uma classe Java com JNI. ................74 


\section{LISTA DE TABELAS}

Tabela 1 - Mensagens de gerenciamento de rede........................................45

Tabela 2 - Mensagens de diagnóstico de rede. .........................................46

Tabela 3 - Informações sobre o proxy agente.............................................50

Tabela 4 - Informações gerais sobre os dispositivos. ....................................51

Tabela 5 - Informações de diagnóstico dos dispositivos.................................52

Tabela 6 - Lista de classes que implementam os objetos da MIB LON-NODE

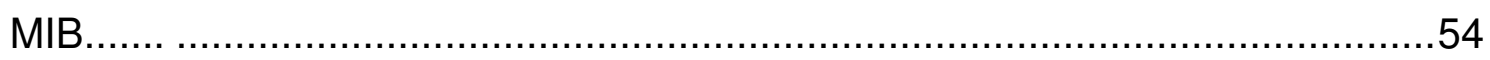




\section{LISTA DE ABREVIATURAS E SIGLAS}

$\begin{array}{ll}\text { ANSI } & \text { American National Standards Institute } \\ \text { API } & \text { Application Programming Interface } \\ \text { ASN.1 } & \text { Abstract Syntax Notation One } \\ \text { CEA } & \text { Consumer Electronics Association } \\ \text { CLNS } & \text { OSI Connectionless Network Service } \\ \text { CLP } & \text { Controlador Lógico Programável } \\ \text { CRC } & \text { Cyclic Redundancy Check } \\ \text { DCS } & \text { Distributed Control System } \\ \text { DDP } & \text { AppleTalk Datagram-Delivery Protocol } \\ \text { DLL } & \text { Dynamic Link Library } \\ \text { EIA } & \text { Electronic Industries Alliance } \\ \text { FAPESP } & \text { Fundação de Amparo à Pesquisa do Estado de São Paulo } \\ \text { GNU } & \text { GNU's Not Unix } \\ \text { IAB } & \text { Internet Arquiteture Board } \\ \text { IDE } & \text { Integrated Development Environment } \\ \text { IETF } & \text { Internet Engineering Task Force } \\ \text { IP } & \text { Internet Protocol } \\ \text { IPX } & \text { Novell Internet Packet Exchange } \\ \text { ISO } & \text { International Organization for Standardization } \\ \text { JDK } & \text { Java Development Kit } \\ \text { JNI } & \text { Java Native Interface } \\ \text { kbps } & \text { 1000 bits por segundo } \\ \text { LAN } & \text { Local Area Network } \\ \text { LON } & \text { Local Operation Network } \\ \text { LTA } & \text { LonTalk Adapter } \\ \text { MAC } & \text { Medium Access Control } \\ \text { MIB } & \text { Management Information Base } \\ \text { NIC-IP } & \text { Network Interface Card - Internet Protocol } \\ & \end{array}$




$\begin{array}{ll}\text { NMS } & \text { Network-Management System } \\ \text { NV } & \text { Network Variable } \\ \text { OID } & \text { Object Identifier } \\ \text { OSI } & \text { Open System Interconnection } \\ \text { PC } & \text { Personal Computer } \\ \text { PDU } & \text { Protocol Data Unit } \\ \text { RFC } & \text { Request for Comment } \\ \text { RMON } & \text { Remote Network Monitoring Management Information Base } \\ \text { SCPT } & \text { Standard Configuration Variable Type } \\ \text { SMI } & \text { Structure of Management Information } \\ \text { SNMP } & \text { Simple Network Management Protocol } \\ \text { SNVT } & \text { Standard Network Variable Type } \\ \text { TCP } & \text { Transmission Control Protocol } \\ \text { TIDIA } & \text { Tecnologia da Informação no Desenvolvimento da Internet } \\ \text { TP/FT } & \text { Avançada } \\ \text { UDP } & \text { Twisted Par Free Topology } \\ \text { UNVT } & \text { User Datagram Protocol } \\ \text { VAV } & \text { User-defined Configuration Variable Type }\end{array}$




\section{SUMÁRIO}

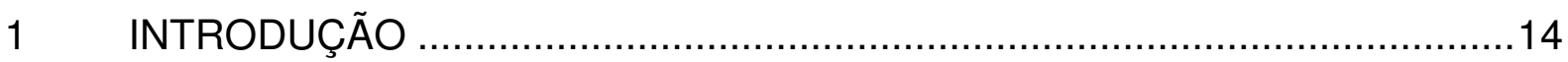

1.1 CONSIDERAÇÕES INICIAIS E MOTIVAÇÃO …….................................14

1.2 OBJETIVO

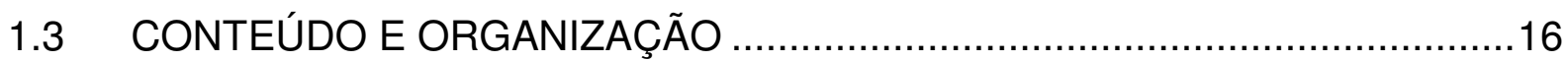

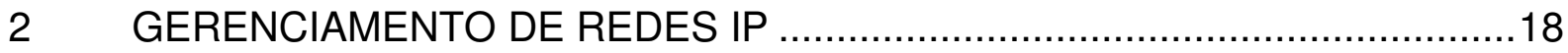

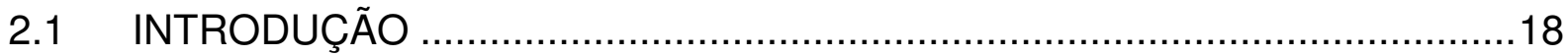

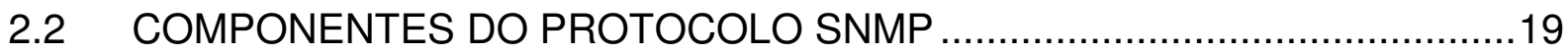

2.2.1 Management Information Base - MIB...............................................19

2.2.2 Structure of Management Information - SMI .......................................20

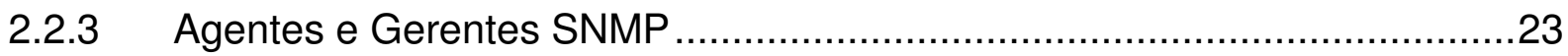

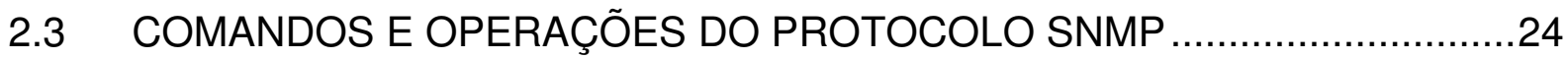

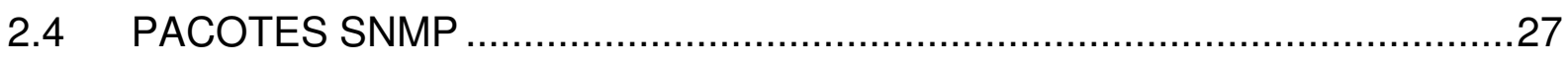

2.5 VANTAGENS E DESVANTAGENS DO PROTOCOLO SNMP ....................29

2.6 UTILIZAÇÃO DO PROTOCOLO SNMP EM REDES DE CONTROLE............30

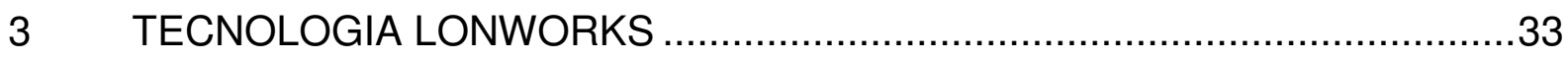

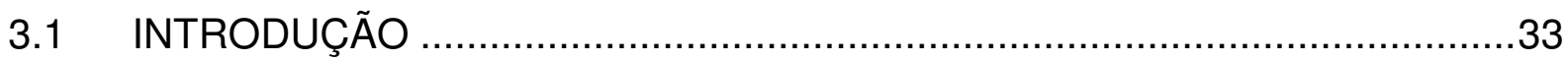

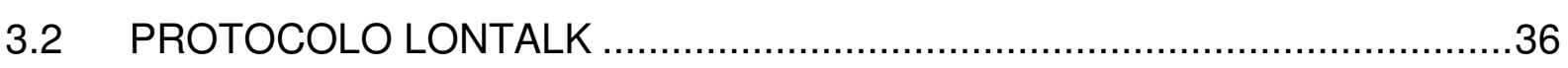

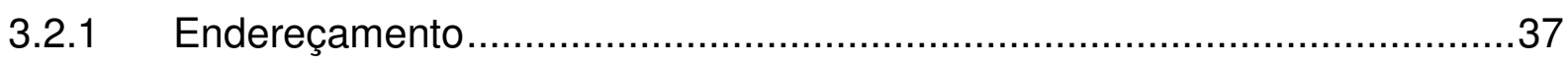

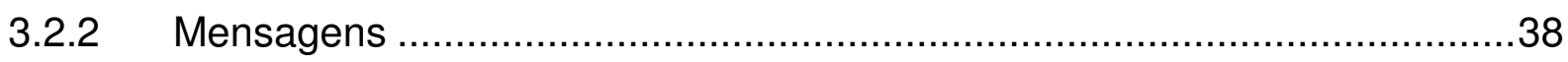

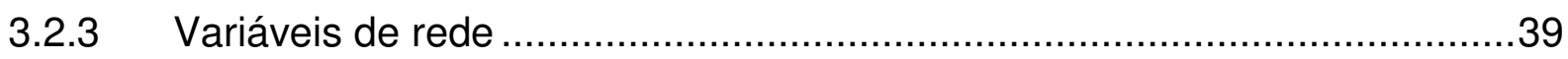

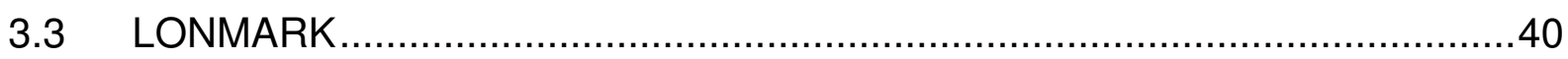

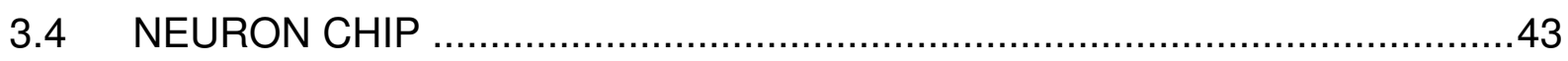

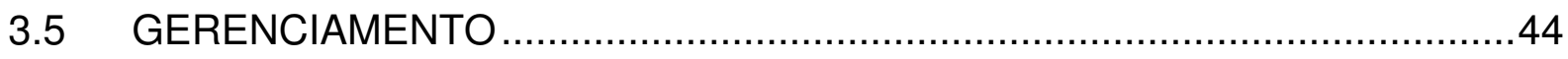

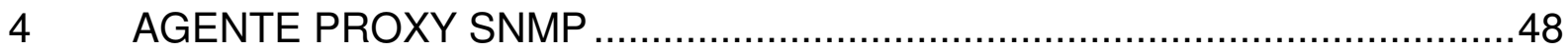

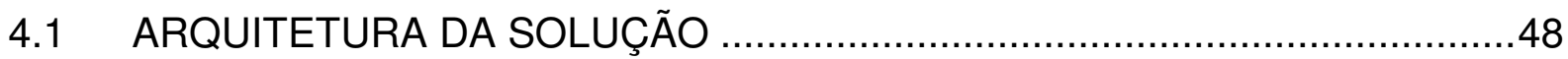

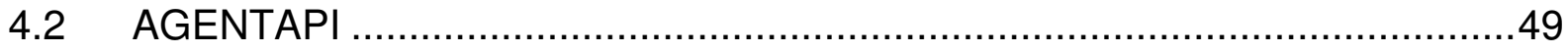

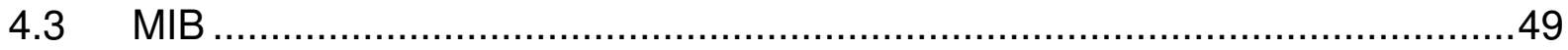

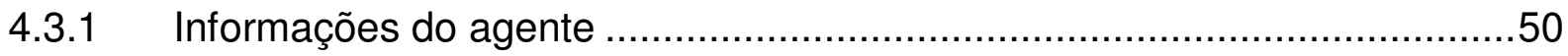

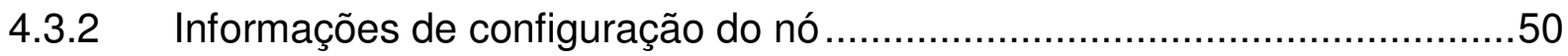

4.3.3 Informações de diagnóstico do nó ..........................................................51

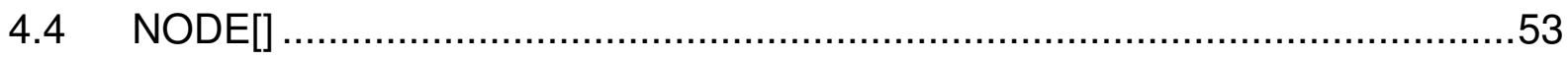

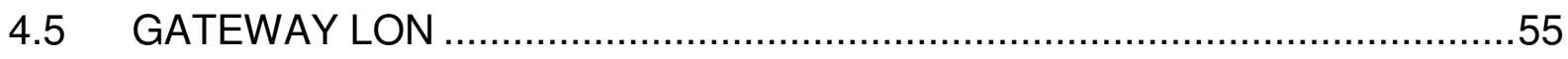




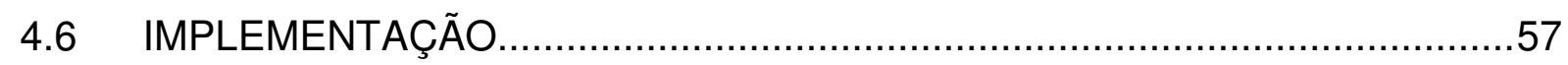

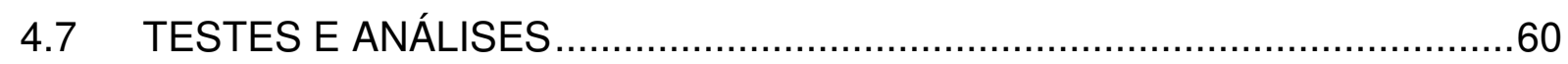

5 DISCUSSÃO DOS RESULTADOS E CONCLUSÃO....................................65

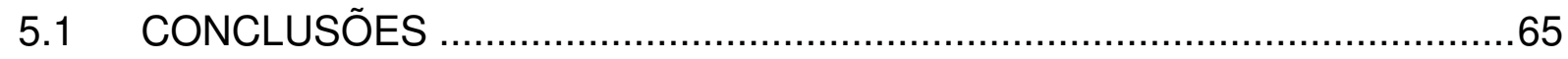

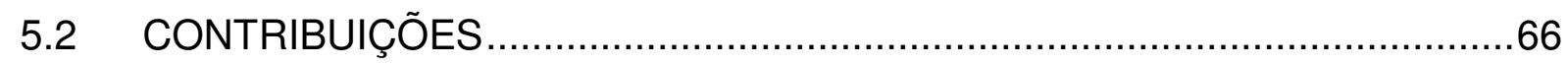

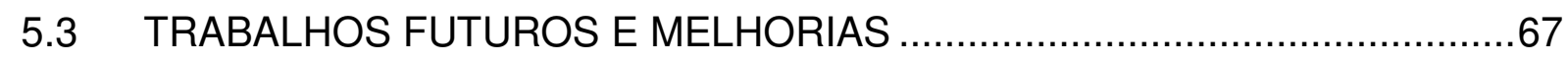

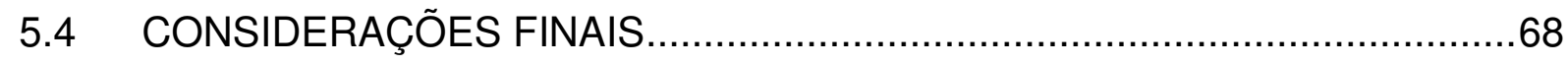

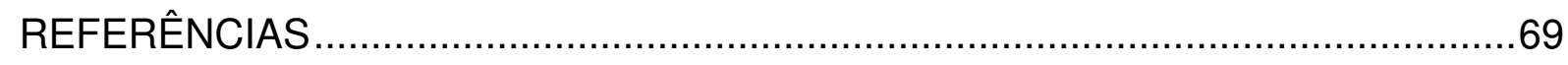

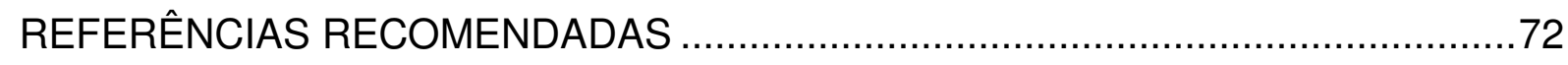

APÊNDICE A - EXEMPLO DE IMPLEMENTAÇÃO JNI .....................................74

A.1 DECLARAR O MÉTODO NATIVO...........................................................

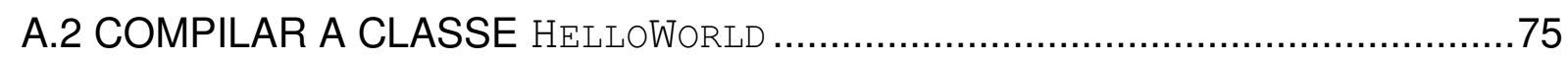

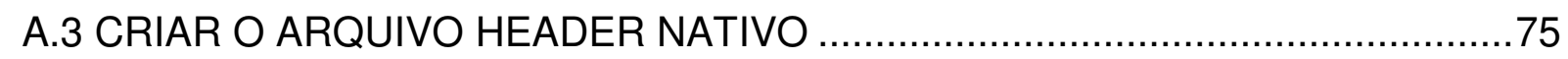

A.4 ESCREVER A IMPLEMENTAÇÃO DO MÉTODO NATIVO..............................76

A.5 COMPILAR O CÓDIGO .C E CRIAR UMA BIBLIOTECA NATIVA .....................77

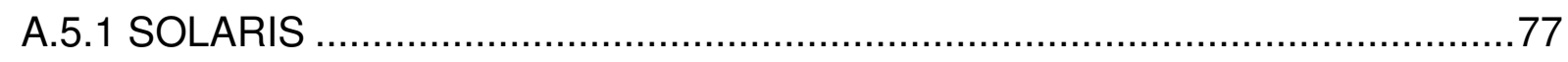

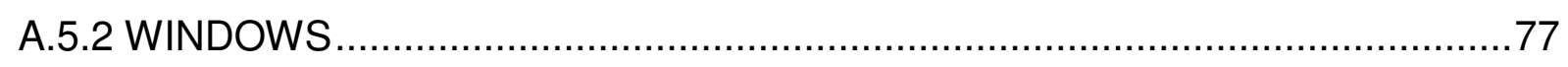

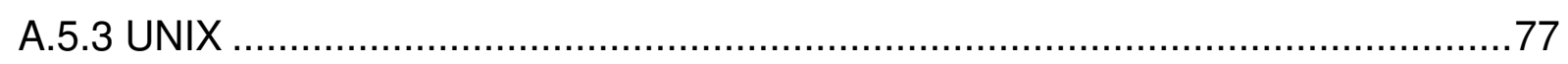

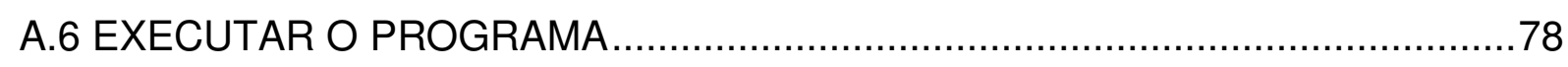

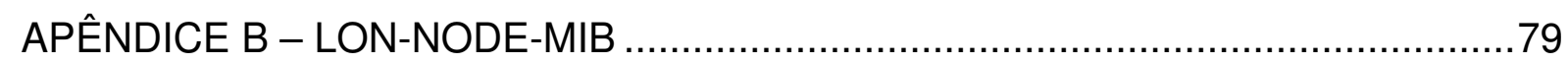




\section{INTRODUÇÃO}

\subsection{CONSIDERAÇÕES INICIAIS E MOTIVAÇÃO}

Os sistemas de automação vêm sendo concebidos, cada vez mais, segundo as topologias de redes de dispositivos para realizar as suas funções. Com isso, uma série de vantagens pode ser apontada: um grande ganho de praticidade de instalação, redução de cabeamento, escalabilidade, dentre outras (CANOVAS; CHERMONT, 2003).

Porém, a falta de um padrão dominante para automação torna cada vez mais difícil a integração entre protocolos e a utilização de uma ferramenta única. No início da década de 90, a empresa americana Echelon criou uma tecnologia para redes de controle denominada LonWorks. O objetivo era interligar um grupo de dispositivos por um barramento, trabalhando em comunicação ponto-a-ponto (peerto-peer) para que pudessem realizar o controle de uma planta. Para isso a Echelon especificou um protocolo de comunicação que trata as sete camadas do modelo Open System Interconnection (OSI), também conhecido como LonTalk. Este protocolo foi encapsulado dentro de um processador, o que permitiu a criação de diferentes dispositivos capazes de se comunicar em rede de uma maneira fácil e eficiente (ECHELON, 1999).

A tecnologia criada já especifica uma série de mensagens de gerenciamento e diagnóstico permitindo que um computador possa ser integrado à rede e seja capaz de verificar a situação de cada um desses dispositivos (CANOVAS, 2006).

No final da década de 90, a Echelon abriu a especificação do protocolo através da norma americana ANSI/EIA/CEA 709.1, o que permitiu que diferentes fabricantes pudessem desenvolver novos dispositivos baseados na tecnologia LonWorks (KASTNER et al., 2005).

Desta forma a aplicação da tecnologia LonWorks para redes de controle se tornou muito atrativa, pois diferentes fabricantes começaram a produzir dispositivos capazes de interagir entre si. Na área de automação predial, a tecnologia LonWorks se apresentou como uma excelente alternativa, sendo cada vez mais utilizada com bons resultados, a ponto de se tornar o padrão oficial europeu para redes de controle prediais (ECHELON, 2007b). 
Paralelamente, as redes de computadores, também conhecidas como Redes IP (Internet Protocol), têm crescido em número e tamanho, tornando cada vez mais necessária a aplicação de um gerenciamento eficiente de recursos da rede. $O$ objetivo principal do gerenciamento de redes é viabilizar o fornecimento de um nível consistente, previsível e aceitável de serviço aos usuários de uma dada rede (LOPES, 2002).

Nesse contexto, no final da década de 80, um grupo subsidiário do Internet Architecture Board (IAB), o Internet Engineering Task Force (IETF), especificou, através de Request for Comment (RFC), um protocolo de comunicação para troca de informações de gerenciamento, o Simple Network Management Protocol (SNMP), como parte do Internet Network Management Framework (KNIZAK et al., 1997).

Devido à sua simplicidade ele passou a ser largamente utilizado por diferentes fabricantes, passando a ser considerado um padrão na área de gerenciamento de redes. O SNMP viabiliza aos administradores o acompanhamento do desempenho, o rastreamento de problemas, o planejamento de crescimento, a avaliação de custos e o gerenciamento de configuração dos recursos da rede.

Atualmente é possível encontrar muitos aplicativos capazes de realizar 0 gerenciamento das redes de computadores através do SNMP. Por outro lado, são poucos os aplicativos disponíveis para gerenciar uma rede LonWorks. Os poucos que encontramos no mercado são de propriedade de algumas empresas que desenvolvem produtos atrelados a tecnologia LonWorks e possuem preços elevados.

Tendo em vista esse cenário, torna-se interessante pensar num mecanismo onde o mesmo sistema de gerenciamento de redes de computadores possa ser utilizado para gerenciar dispositivos de automação.

Assim, uma mesma equipe técnica de gerenciamento pode diagnosticar algum dispositivo da rede de automação que não esteja operando de maneira adequada, da mesma forma que identifica que um roteador da rede corporativa está sobrecarregado.

\subsection{OBJETIVO}

O objetivo deste trabalho de pesquisa foi o desenvolvimento de um agente proxy SNMP para realizar o gerenciamento de uma rede de automação baseada na 
tecnologia LonWorks através de aplicativos comumente utilizados no gerenciamento de redes IP.

Para isso utilizou-se uma Application Programming Interface (API) desenvolvida pelo Professor Rui Pedro Lopes do Instituto Politécnico de Bragança Portugal (LOPES; OLIVEIRA, 2003), por ela já implementar toda a estrutura de comunicação de um agente SNMP. A API foi disponibilizada em forma de um package Java, chamado AgentAPI, regido pela licença GNU's Not Unix (GNU). Ela implementa a modelagem do agente SNMP e suas tarefas comuns, facilitando o processo de desenvolvimento.

Definiu-se também uma Management Information Base (MIB) para organizar as informações de diagnóstico provenientes de um número arbitrário de dispositivos LonWorks.

Conseqüentemente, foi possível, através desse trabalho desenvolver uma interface Java capaz de se comunicar com a rede LonWorks, o que permite que novos trabalhos desenvolvidos possam se utilizar desta interface em suas aplicações, em especial os trabalhos que vêm sendo desenvolvidos pelo grupo de pesquisa do LAA (2006).

\subsection{CONTEÚDO E ORGANIZAÇÃO}

Esse texto apresenta o resultado do trabalho de mestrado, sendo que nesse primeiro capítulo foi apresentada uma introdução do trabalho, seu objetivo e sua motivação.

O segundo capítulo apresenta um breve resumo sobre o protocolo SNMP, onde se destacam alguns pontos que foram relevantes para a realização do trabalho. No final do capítulo são apresentadas algumas aplicações do SNMP em automação e algumas arquiteturas utilizadas, que servirão como orientação para a realização das pesquisas associadas a esse trabalho.

O terceiro capítulo descreve resumidamente os conceitos da tecnologia LonWorks, sendo finalizado com destaque nas características de gerenciamento inerentes a tecnologia e utilizadas para a realização do trabalho.

No quarto capítulo detalha-se o desenvolvimento do agente, a arquitetura utilizada e alguns testes realizados. É neste capítulo que se concentram as informações relativas à implementação do agente e suas principais características. 
O trabalho é encerrado no quinto capítulo, com as conclusões relativas ao agente desenvolvido, as contribuições e algumas melhorias que podem ser implementadas.

No final deste documento encontram-se as referências bibliográficas utilizadas e outras recomendadas, e também dois apêndices, onde o primeiro apresenta um roteiro para a utilização de Dynamic Link Library $(D L L)$ em programas escritos em linguagem Java, e o segundo apêndice traz a Management Information Base (MIB) criada para esse trabalho. 


\section{GERENCIAMENTO DE REDES IP}

\subsection{INTRODUÇÃO}

O SNMP apresenta-se como um protocolo da camada de aplicação que facilita o intercâmbio de informações de gerenciamento entre dispositivos de uma rede de computadores (CISCO, 2006a). Ele se insere sobre a pilha de protocolos Transmission Control Protocol / Internet Protocol (TCP/IP), tornando-se um padrão para a monitoração de elementos de interconexão de redes baseadas em Local Área Network (LAN), tais como impresssora, pontes (bridges) e roteadores (routers). O SNMP viabiliza aos administradores o acompanhamento do desempenho, 0 rastreamento de problemas, o planejamento de crescimento, a avaliação de custos e o gerenciamento de configuração dos recursos da rede.

Segundo Stallings (1996), o SNMP não é apenas um protocolo e sim um conjunto de especificações de gerenciamento, incluindo: o protocolo propriamente dito, as definições de estruturas de dados e outros conceitos associados.

Atualmente existem três versões do SNMP:

- SNMP versão 1 (SNMPv1);

- $\quad$ SNMP versão 2 (SNMPv2);

- $\quad$ SNMP versão 3 (SNMPv3).

O SNMPv1 foi a descrição inicial do protocolo SNMP, através da RFC 1157, prevendo a sua operação sobre os protocolos User Datagram Protocol (UDP), IP, OSI Connectionless Network Service (CLNS), AppleTalk Datagram-Delivery Protocol (DDP) e Novell Internet Packet Exchange (IPX) (CISCO, 2006a). Apesar de sua facilidade de implementação, esta versão apresentava diversos problemas relacionados à segurança do gerenciamento. Por sua vez, o SNMPv2, descrito pelas RFCs 1441 a 1450, preocupou-se em oferecer melhorias em segurança e funcionalidade, apesar de apresentar, ainda assim, falhas nas áreas de autenticação, encriptação de mensagens e na própria segurança. O SNMPv3, por fim, através das RFCs 2271 a 2275, além de ser compatível com suas versões anteriores, incluiu ao protocolo SNMP o controle de acesso, a autenticação e a manutenção da privacidade da informação gerenciada (MAURO; SCHMIDT, 2005). 


\subsection{COMPONENTES DO PROTOCOLO SNMP}

\subsubsection{Management Information Base - MIB}

A Management Information Base (MIB) é um conjunto de informações provenientes da rede gerenciada organizadas hierarquicamente. Tais informações são guardadas em um banco de dados de objetos gerenciados que podem ser acessados através de protocolos de gerenciamento de redes tais como o SNMP.

Para identificar unicamente um objeto gerenciado na hierarquia da MIB, usase o identificador de objetos (object identifier, ou object ID). Essa identificação não ambígua e universal de objetos arbitrários é feita através de uma árvore hierárquica de objetos MIB (Figura 1), baseado no Esquema de Identificação de Objetos (Object Identification Scheme) definido pelo OSI (RAD, 2006).

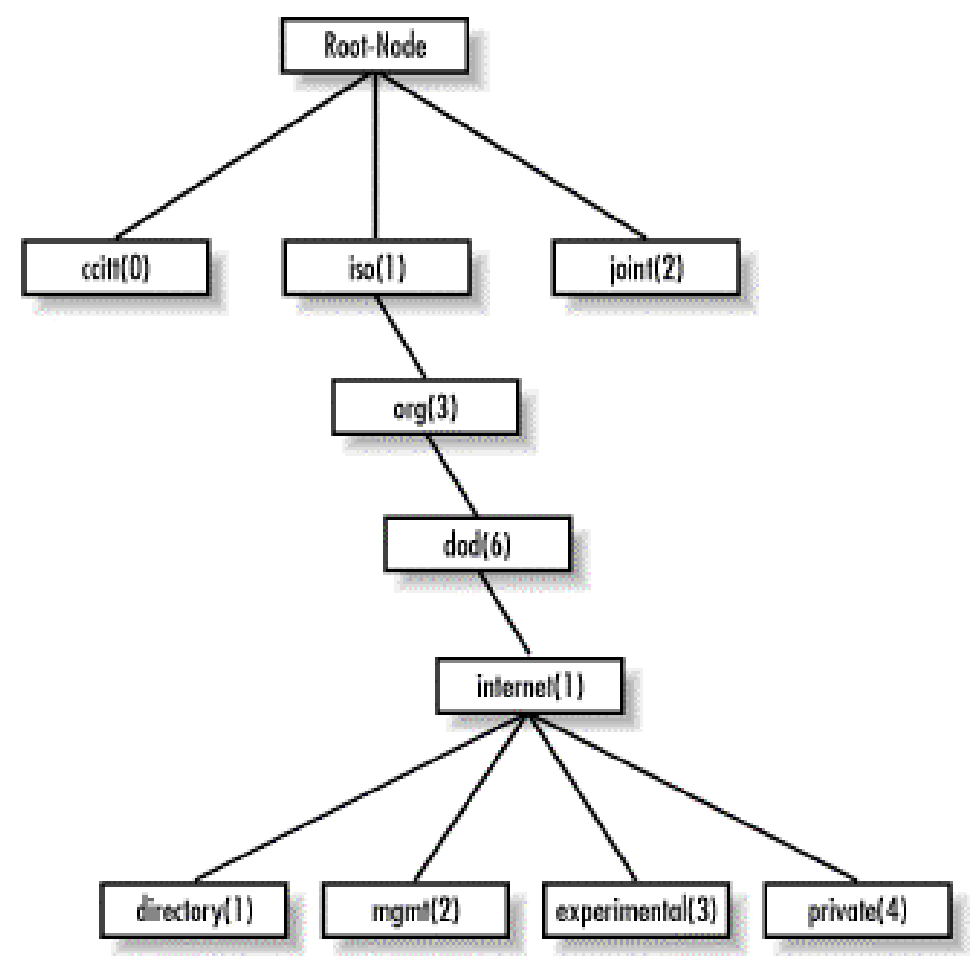

Figura 1. Estrutura de nomenclatura de objetos no SNMP. Extraído de Mauro; Schmidt (2005).

Um objeto gerenciado, que pode ser chamado de objeto MIB, objeto ou simplesmente MIB, é uma entre diversas características de um elemento gerenciado. Eles são compostos por uma ou mais instâncias de objeto que, essencialmente, são variáveis. Um objeto MIB, por exemplo, pode guardar o valor associado ao número de pacotes que chegaram a um dispositivo desde a última 
reinicialização do sistema, ou mesmo o estado administrativo específico de um equipamento. Os valores das instâncias dos objetos podem ser guardados de forma escalar ou tabular. Enquanto um objeto escalar define uma única instância de objeto, como por exemplo, a descrição do agente, um objeto tabular define um grupo de instâncias de objeto, que são encontrados em tabelas MIB, que podem representar, por exemplo, uma lista de interfaces de rede (CISCO, 2006a).

Há duas especificações para os objetos monitorados no gerenciamento de redes, a primeira é denominada MIB-I, enquanto que a segunda, e sua sucessora, é denominada MIB-II.

A MIB-I teve como objetivo incluir um número mínimo de objetos gerenciados, para torná-la mais atrativa aos olhos dos fabricantes, foi estabelecido um limite de aproximadamente 100 objetos nessa primeira fase.

Dentro da especificação da MIB-I foram seguidos alguns critérios que o objeto MIB deveria atender, por exemplo:

- Ser essencial para a análise de falhas ou configuração;

- Ter uma designação única;

- Ter utilidade evidente;

- Ser suficientemente geral por natureza, não dependente de equipamentos ou fabricantes.

Além disso, os objetos descritos não deveriam ser facilmente derivados de outros e sua estrutura abstrata precisava ser universal, permitindo extensões privadas.

A MIB-I sofreu modificações e evoluiu para a MIB-II. Esta, por sua vez, devia manter a compatibilidade com sua antecessora e, adicionalmente incluir objetos para refletir novos requisitos operacionais, permitir um melhor suporte a equipamentos multi-protocolo e ter um texto melhor elaborado para fins de clareza.

O número de objetos presentes na MIB-II passou a ser de aproximadamente 170. Com as melhorias adquiridas a maioria dos fabricantes, hoje em dia, implementa a MIB-II em seus equipamentos (MAURO; SCHMIDT, 2005).

\subsubsection{Structure of Management Information - SMI}

A Structure of Management Information (SMI) define regras para a descrição de informações de gerenciamento usando a notação Abstract Syntax Notation One 
(ASN.1). Ela especifica a estrutura que dá nomes únicos a cada objeto gerenciado na rede e também quais tipos de dados são permitidos na MIB. A SMI faz três especificações chaves: tipos de dados ASN.1, tipos de dados específicos da SMI e tabelas MIB SNMP (CISCO, 2006a).

\subsubsection{SNMPV1}

A SMI do SNMPv1 especifica que todos os objetos gerenciados devem conter obrigatoriamente certo subconjunto de tipos de dados ASN.1 associados aos mesmos. Dessa forma, os três tipos de dados requeridos são:

- Nome (name): é o identificador do objeto (object identifier);

- Sintaxe (syntax): define o tipo de dado do objeto (por exemplo: inteiro ou cadeia de caracteres), sendo este um subconjunto da sintaxe definida para o ASN.1;

- Codificação (encoding): descreve como a informação associada ao objeto gerenciado é posta na forma de uma série de dados para transmissão na rede.

Os tipos de dados específicos da SMI descritos na SMI do SNMPv1 são divididos em duas categorias: tipos de dados simples (simple data types) e tipos de dados de aplicação (application-wide data types).

Os tipos de dados simples correspondem a valores únicos. São eles:

- Inteiros (integers): inteiros com sinal, no intervalo de -2.147.483.648 a 2.147.483.647;

- Octetos (octets): seqüências ordenadas de 0 a 65.535 octetos;

- Strings: Cadeias de caracteres;

- Identificadores de objetos (object identifiers): provenientes de um conjunto de todos os identificadores de objetos alocados de acordo com as regras especificadas no ASN.1.

Por sua vez, os tipos de dados de aplicação definidos são:

- Endereços de rede (network addresses): endereços de famílias de protocolos particulares (restritos a 32 bits);

- Contadores (counters): inteiros não negativos que podem ser incrementados até atingirem um valor máximo, retornando então a zero (restritos a 32 bits); 
- Medidores (gauges): inteiros não negativos que podem ser incrementados ou decrementados, mas que retêm, na verdade, o máximo valor atingido pela variável;

- Períodos de tempo (time ticks): representam o tempo decorrido após determinado evento, em centésimos de segundo;

- Opaques: codificação arbitrária para transmitir informações por meio de strings, que não se encaixam nos tipos de dados usados pelo SMl;

- Inteiros (integers): inteiros com sinal (redefine o tipo de dados "inteiro", que tem precisão arbitrária no ASN.1 mas precisão limitada no SMI);

- Inteiros sem sinal (unsigned integers): muito usual quando os valores são sempre não negativos.

Por fim, a SMI do SNMPv1 define também tabelas fortemente estruturadas, as quais são usadas para agrupar instâncias de um objeto tabular, ou seja, um objeto que contém múltiplas variáveis. Tais tabelas são compostas por zero ou mais linhas, as quais são indexadas de forma a permitir que o SNMP recupere ou altere uma linha inteira com um simples comando Get, GetNext ou Set (CISCO, 2006a).

\subsubsection{SNMPV2}

A SMI do SNMPv2 faz adições e melhorias aos tipos de dados específicos do SNMPv1.

- Cadeias de bits (bit strings): zero ou mais bits com nomenclatura, que especificam um determinado valor;

- Endereços de rede (network addresses): endereços de famílias particulares de protocolos, abrangendo desta vez mais protocolos que no caso do SNMPv1;

- Contadores (counters): inteiros não negativos que podem ser incrementados até atingirem um valor máximo, retornando então a zero (passam de 32, no SNMPv1, a 64 bits).

Além disso, especifica o conceito de módulos de informação, que detalha um grupo de definições relacionadas. Existem três tipos de módulos de informação SMI:

- Módulos MIB: que contêm descrições de objetos gerenciados interrelacionados; 
- Declarações de compatibilidade: as quais se apresentam como um modo sistemático de descrever um grupo de objetos gerenciados que devem ser implementados conforme um determinado padrão;

- Declarações de capacidade: que indicam o nível preciso de suporte de um determinado agente em relação a um grupo MIB.

\subsubsection{Agentes e Gerentes SNMP}

Os objetos gerenciados pelo SNMP, conforme visto no tópico 2.2.1, são arranjados em um banco de dados virtual, chamado de base de informações gerenciais ou MIB. Tais objetos podem ser de fato, hardwares, parâmetros de configuração ou estatísticas de desempenho, por exemplo, relacionados diretamente à operação de um determinado dispositivo de rede.

Do ponto de vista de arquitetura, uma rede gerenciada através do SNMP é composta por três elementos chaves: dispositivos gerenciados, agentes e sistemas de gerenciamento da rede, conhecidos também como Network-Management Systems (NMS) ou gerentes (Figura 2). O SNMP viabiliza a comunicação entre agentes e gerentes para que os objetos correspondentes aos diversos dispositivos de rede possam ser acessados e modificados quando necessário. A seguir, detalharemos as principais funcionalidades de cada um desses elementos.

Os dispositivos gerenciados são pontos da rede que contêm agentes SNMP. Esses equipamentos coletam e armazenam informações de gerenciamento em um banco de dados composto pelos objetos descritos na MIB, tornando-as disponíveis aos gerentes por meio do uso do protocolo SNMP. Os dispositivos gerenciados também chamados de elementos de rede - podem ser: servidores de acesso, concentradores (hubs), pontes (bridges), switches, roteadores (routers), computadores (hosts) ou até mesmo impressoras. 


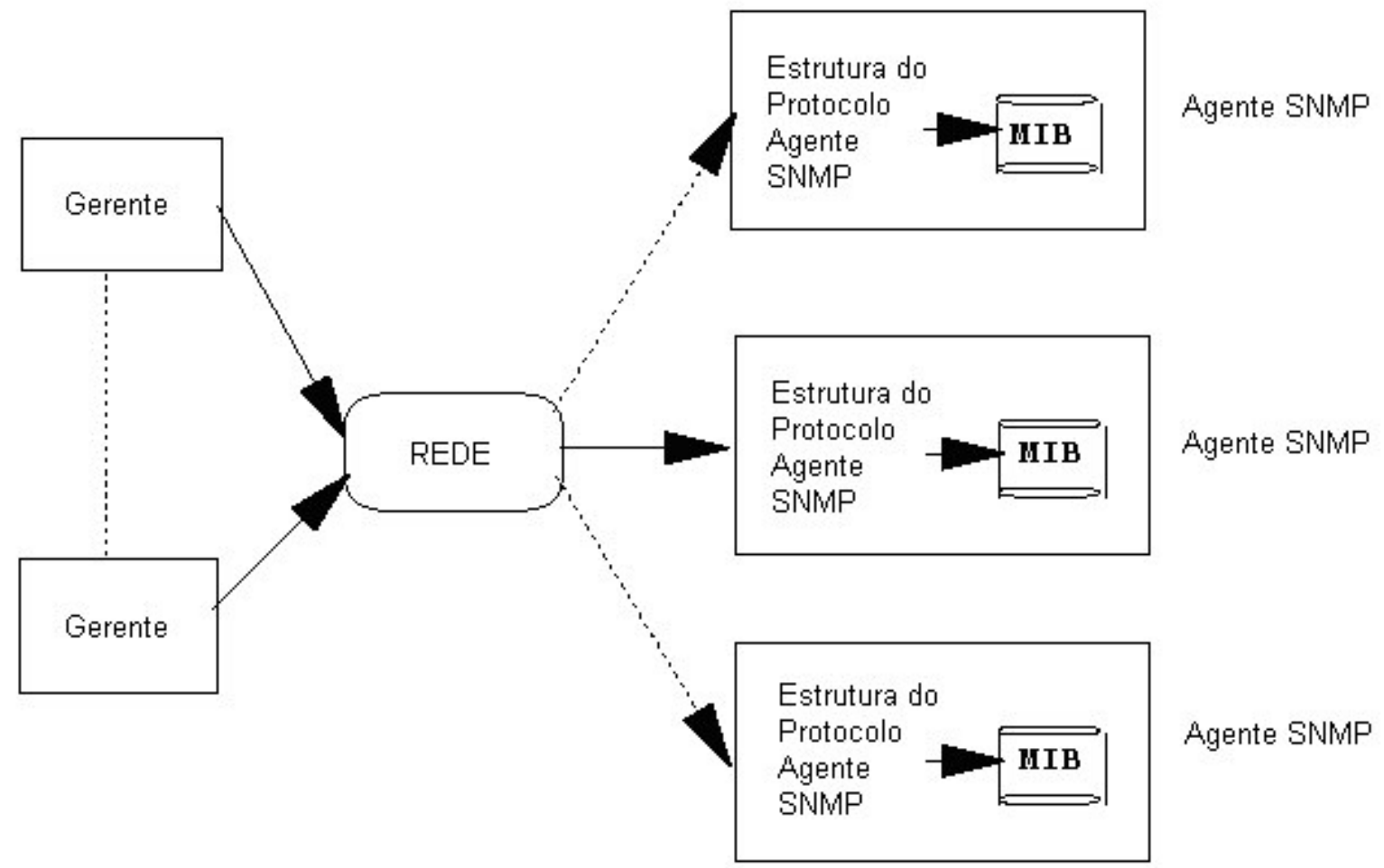

Figura 2. Arquitetura da Rede SNMP. Baseado em RAD (2006).

Por outro lado, os gerentes SNMP cumprem a função de executar aplicações de gerenciamento de rede que monitoram e controlam os dispositivos gerenciados. Eles provêm a grande parte do processamento e dos recursos de memória necessários para o gerenciamento de redes. Em qualquer rede SNMP pode haver um ou mais destes elementos (MAURO; SCHMIDT, 2005).

Finalmente, agentes SNMP consistem em módulos de software para gerenciamento de redes que residem em um dispositivo gerenciado. $O$ agente tem conhecimento das informações locais relevantes para o gerenciamento, sendo sua função a tradução dessas informações em uma forma compatível com o SNMP. Dessa forma, o agente fornece as informações contidas na MIB do dispositivo às aplicações gerentes, além de viabilizar a alteração de configurações ou parâmetros do dispositivo quando solicitado.

\subsection{COMANDOS E OPERAÇÕES DO PROTOCOLO SNMP}

Para permitir que os gerentes acessem e modifiquem as MIBs dos equipamentos gerenciados, o SNMP, sendo um protocolo da camada de aplicação, oferece comandos básicos para a realização de tais funções. São eles: 
- Read: possibilita ao sistema gerenciador a monitoração de dispositivos gerenciados através da viabilização da verificação das diferentes variáveis mantidas no equipamento;

- Write: permite ao gerente o controle sobre os dispositivos de rede por meio da modificação dos valores das variáveis armazenadas nos mesmos;

- Transversal operations: possibilitam ao gerenciador do sistema a coleta seqüencial de informações encontradas em tabelas de variáveis (como tabelas de roteamento, por exemplo);

- Trap: é usado pelos agentes SNMP que desejam reportar eventos assincronamente ao gerenciador do sistema: se o dispositivo gerenciado necessita alertar ao gerente algo significante referente ao seu estado na rede, o mesmo utiliza o comando trap.

Para implementar os comandos de aplicação, o SNMP, sendo um protocolo simples de requisições e respostas (request/response), utiliza-se de operações fundamentais, tais como GetRequest, GetResponse, GetNextRequest, SetRequest e Trap:

- GetRequest: usada pelos gerentes para recuperar o valor de uma ou mais instâncias de objetos de um agente. Se o agente, respondendo à operação GetRequest, não puder fornecer valores para todas as instâncias de objetos especificadas, o mesmo não provê valor algum;

- GetResponse: mecanismo utilizado pelo agente SNMP para atender a solicitação de leitura ou escrita feita pelo gerente;

- SetRequest: empregada pelos gerentes para efetuar modificações nos valores das instâncias de objetos em um agente;

- GetNextRequest: utilizada pelos sistemas gerenciadores para a obtenção do valor da próxima instância em um objeto tabular presente em um agente;

- Trap: usada pelos agentes para informar, assincronamente, eventos significativos aos sistemas gerenciadores.

$\mathrm{Na}$ Figura 3 são ilustradas as possíveis trocas de informações entre o gerente e o agente.

O SNMPv2 adiciona e melhora algumas operações de protocolo em relação ao SNMPv1. A operação Trap do SNMPv2, por exemplo, serve à mesma função do 
Trap descrito no SNMPv1, mas utiliza-se de um formato de mensagem diferente e está destinada a substituir sua antecessora. O SNMPv2 também define duas novas operações de protocolo: GetBulk e Inform.

A operação GetBulk é usada pelos gerentes SNMP para recuperar eficientemente grandes blocos de dados, como múltiplas linhas em uma tabela. No SNMPv2, se o agente respondendo a um GetBulk não puder fornecer valores para todas as variáveis da lista, ele provê ao gerente resultados parciais.

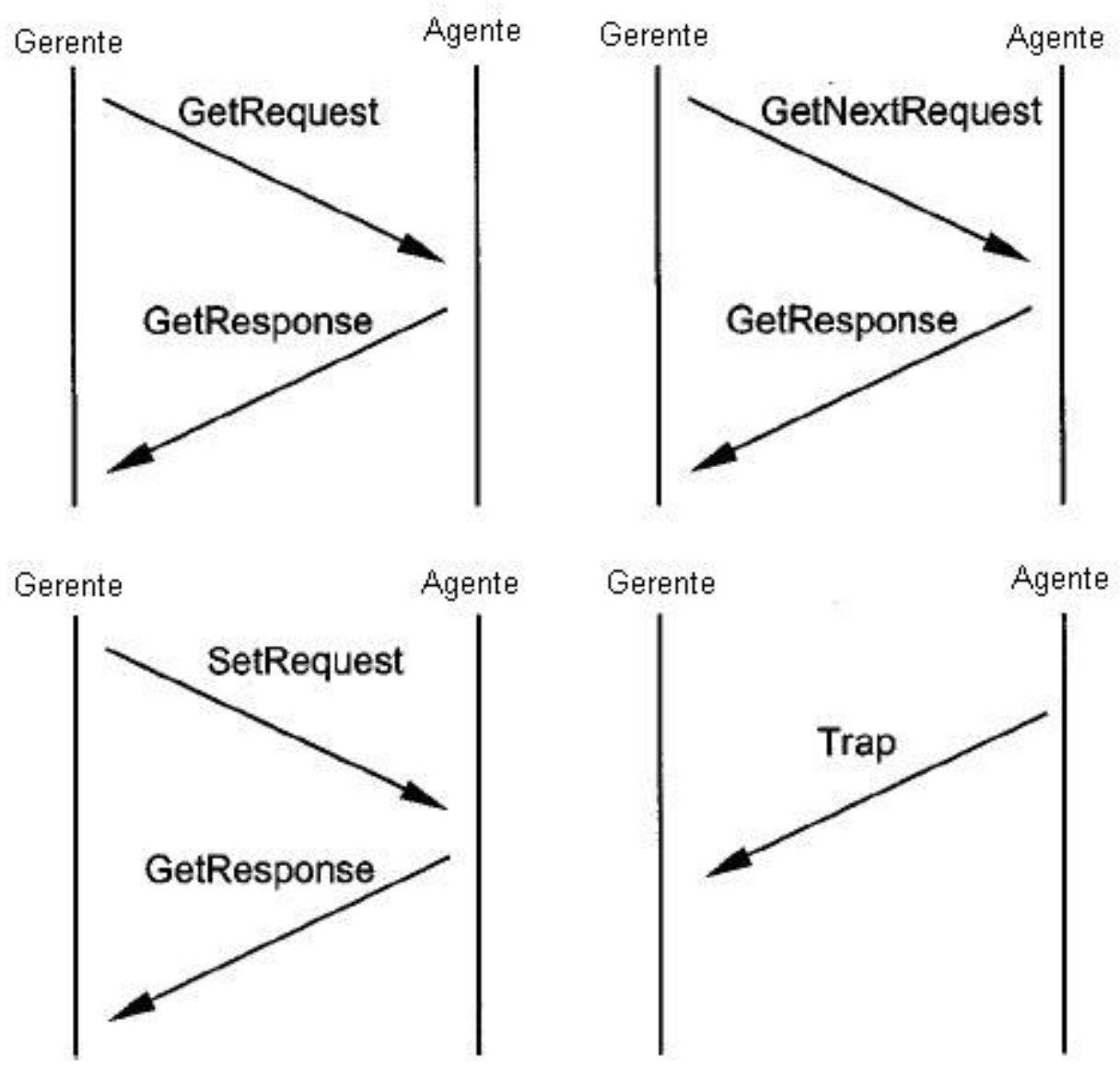

Figura 3. Operações do protocolo SNMP. Baseado em Kunes; Sauter (2001).

Por sua vez, a operação Inform possibilita que um sistema gerenciador envie informações semelhantes a um "trap" a outro sistema gerenciador, e receba a resposta correspondente a essa operação. Através do uso da mesma, portanto, dissolve-se o conceito de gerenciamento centralizado presente fortemente no SNMPv1, um sistema passa a poder atuar exclusivamente tanto como gerente quanto como agente, ou pode implementar as duas funções ao mesmo tempo. Quando um sistema opera tanto como gerente quanto como agente, outro sistema 
gerenciador pode requisitar que tal gerente controle certos dispositivos e providencie um resumo dos informações apreendidas (CISCO, 2006b).

\subsection{PACOTES SNMP}

Os formatos das mensagens do protocolo do SNMP são divididos em duas partes: o cabeçalho da mensagem (header) e o Protocol Data Unit (PDU) (Figura 4). As mensagens são encaminhadas através do protocolo de transporte UDP.

\begin{tabular}{|c|c|c|}
\hline version & community & SNMP PDU \\
\hline
\end{tabular}

Mensagem SNMP

\begin{tabular}{|l|l|l|l|l|}
\hline PDU type & request-id & 0 & 0 & variable-bindings \\
\hline
\end{tabular}

GetRequest PDU, GetNextRequest PDU e SetRequest PDU

\begin{tabular}{|l|l|l|l|l|}
\hline PDU type & request-id & error-status & error-index & variable-bindings \\
\hline
\end{tabular}

GetResponse PDU

\begin{tabular}{|l|l|l|l|l|l|l|}
\hline PDU type & enterprise & agent-addr & generic-trap & specific-trap & time-stamp & variable-bindings \\
\hline
\end{tabular}
Trap PDU

\begin{tabular}{|l|l|l|l|l|l|l|} 
name 1 & value 1 & name 2 & value 2 & $\ldots$ & name $\mathrm{n}$ & value $\mathrm{n}$ \\
\hline
\end{tabular}
variable-bindings

Figura 4. Formato das mensagens SNMP. Baseado em Lopes (2003).

O cabeçalho da mensagem contém dois campos:

- Número da Versão (Version number): especifica a versão do SNMP usada;

- Nome da Comunidade (Community name): define um ambiente de acesso para um grupo de gerentes. Gerentes que pertencem à mesma comunidade são ditos para existir no mesmo domínio administrativo. As comunidades servem de certa forma como uma autenticação fraca, pois impossibilitam que dispositivos que não sabem o nome da comunidade realizem as operações SNMP.

Os SNMP PDUs contêm os comandos específicos (GetRequest, SetRequest, etc.) e informações dos objetos envolvidos na transação. Os campos do SNMP PDU tem comprimento variável, como descrito pela ASN.1. A Figura 4 ilustra 
os campos das transações GetRequest, GetNextRequest, GetResponse e SetRequest. Abaixo descrevemos cada um dos campos disponíveis:

- Tipo de PDU (PDU Type): especifica o tipo de PDU transmitido;

- Identificador de Requisição (Request ID): identificador que associa uma requisição SNMP com uma resposta;

- Erro Status (Error status): Indica o número do erro e o tipo de erro. Somente a operação GetResponse preenche este campo, as outras operações preenche o campo com 0;

- Índice do Erro (Error index): associa um erro com uma instância particular do objeto. Somente a operação GetResponse preenche este campo, as outras operações preenche o campo com 0;

- Variáveis (Variable bindings): campo de dados do PDU. Possui cada variável associada a uma instância do objeto com o seu respectivo valor. Nos comandos GetRequest e GetNextRequest os valores são ignorados.

A operação Trap possui um formato de PDU distinto, com os seguintes campos:

- Empresa (Enterprise): identifica o tipo de objeto gerenciado que gerou o trap;

- Endereço do Agente (Agent address): fornece o endereço do objeto gerenciado que gerou o trap;

- Tipo Genérico de Trap (Generic Trap Type): indica o número de um tipo genérico de trap;

- Tipo Específico de Trap (Specific Trap Code): indica o número de um tipo especifico de trap;

- Tempo (Time Stamp): fornece o tempo entre a última reinicialização da rede e o momento da geração do trap;

- Variáveis (Variable Bindings): campo de dados do PDU. Possui cada variável associada a uma instância do objeto com o seu respectivo valor (CISCO, 2006a). 


\subsection{VANTAGENS E DESVANTAGENS DO PROTOCOLO SNMP}

A implementação de uma rede compatível com SNMP oferece significativos benefícios. O primeiro deles, sem dúvida, é a possibilidade de administração, monitoração e controle eficaz de uma rede, de forma a se prevenir, detectar e corrigir falhas, além da própria manutenção de um desempenho satisfatório na mesma.

Por ser extremamente simples, o SNMP requer pouca codificação para ser implementado. Desta forma, fabricantes podem facilmente acoplar agentes SNMP em seus produtos permitindo que novos dispositivos possam ser gerenciados.

O SNMP é extensível, o que permite que os fabricantes adicionem funcionalidades de gerenciamento de rede a produtos já existentes. Ele oferece uma separação adequada da arquitetura de gerenciamento em relação à arquitetura dos dispositivos de hardware, possibilitando a ampliação da base de equipamentos que o suportam, independentemente de fabricante.

Ao mesmo tempo o SNMP permite, por oferecer padronização, que diferentes produtos de rede sejam gerenciados por uma mesma aplicação de gerenciamento, provendo aos nós da rede a interoperabilidade desejada.

Além disso, a independência de operação entre gerentes e agentes torna a rede mais robusta, um gerente deve continuar a funcionar mesmo se um agente falhar.

Outra vantagem é a eficiência na entrega dos pacotes do SNMP devido à comum utilização do UDP como protocolo de transporte que resulta em uma menor sobrecarga de processamento.

Por fim, o uso de agentes, denominados agente proxy SNMP, que se fazem passar por integrantes de outras redes, permite a integração de outras arquiteturas de gerenciamento ao SNMP (RAD, 2006), sendo este trabalho um exemplo dessa aplicação.

Apesar de todos esses pontos positivos, vale lembrar algumas das desvantagens relativas à escolha do protocolo SNMP para o gerenciamento de redes.

Devido ao uso do UDP como protocolo de transporte mais comum para o SNMP aumenta-se a probabilidade de perdas de dados, por esse tipo de protocolo de transporte não realizar uma verificação de entrega e não ter nenhum tipo de mecanismo para retransmissão de pacote, apesar de diminuir a sobrecarga de 
processamento no envio e recebimento de pacotes. Aliado à prática de polling sobre os elementos de rede gerenciados, fato que pode revelar-se preocupante em grandes redes de gerenciamento centralizado, o protocolo SNMP pode tornar-se excessivamente ineficiente, por utilizar de forma abusiva, os recursos computacionais disponíveis.

Apesar disso, o uso do padrão Remote Network Monitoring MIB (RMON) é uma opção para que tal problema seja contornado, pois consiste em uma MIB que permite a certos dispositivos a monitoração local de outros equipamentos, o que diminui o tráfego agente-gerente pelo envio somente dos dados e eventos mais relevantes resumidos ao sistema gerenciador e o uso de gerenciamento distribuído, viabilizado pela operação de protocolo Inform, disponível a partir do SNMPv2.

Por último, a questão de segurança revela-se preocupante no SNMPv1 e SNMPv2, nenhum dos dois provê funcionalidades adequadas nesse quesito, utilizando-se de artifícios muito vulneráveis para o controle das mensagens, como as comunidades, que são simples conjunto de gerentes aos quais um agente pode ou deve se reportar, e perfis de comunidades, que restringem o acesso a variáveis da MIB e o tipo de acesso a esses dados de acordo com a comunidade a que o outro elemento pertence. O SNMPV3, no entanto, oferece recursos para que seja possível contornar tais desvantagens, ele garante a autenticação e encriptação das mensagens de gerenciamento.

\subsection{UTILIZAÇÃO DO PROTOCOLO SNMP EM REDES DE CONTROLE}

Atualmente existe uma grande quantidade de Controladores Lógicos Programáveis (CLP) e sistemas de controle distribuídos, conhecidos como Distributed Control System (DCS), que possuem dentro deles registros de dados dedicados e que disponibilizam informações em tempo real sobre a performance e o estado de operação do controlador. Um agente SNMP embarcado no CLP pode comunicar-se com um gerente e trocar informações a respeito de suas operações.

A conexão desses dispositivos a outros elementos de rede Ethernet possibilitam que esses dados de operação possam ser acessados e manipulados através de sistemas de gerenciamento de rede baseados no SNMP. Um pacote que provê essa funcionalidade de gerenciamento de redes de automação através da utilização de SNMP em redes TCP/IP Ethernet é o Industrial SNMP, ou iSNMP (INS, 2006). 
Diferentes topologias podem ser utilizadas para interconectar uma rede de controle através do SNMP. Em Knizak et al. (1997) encontramos diferentes abordagens para realizar essa integração, uma delas seria através da implementação de um agente SNMP em cada um dos dispositivos da rede de controle como propõe a INS (2006), porém essa estrutura amplia o cabeamento necessário como pode ser observado na Figura 5.

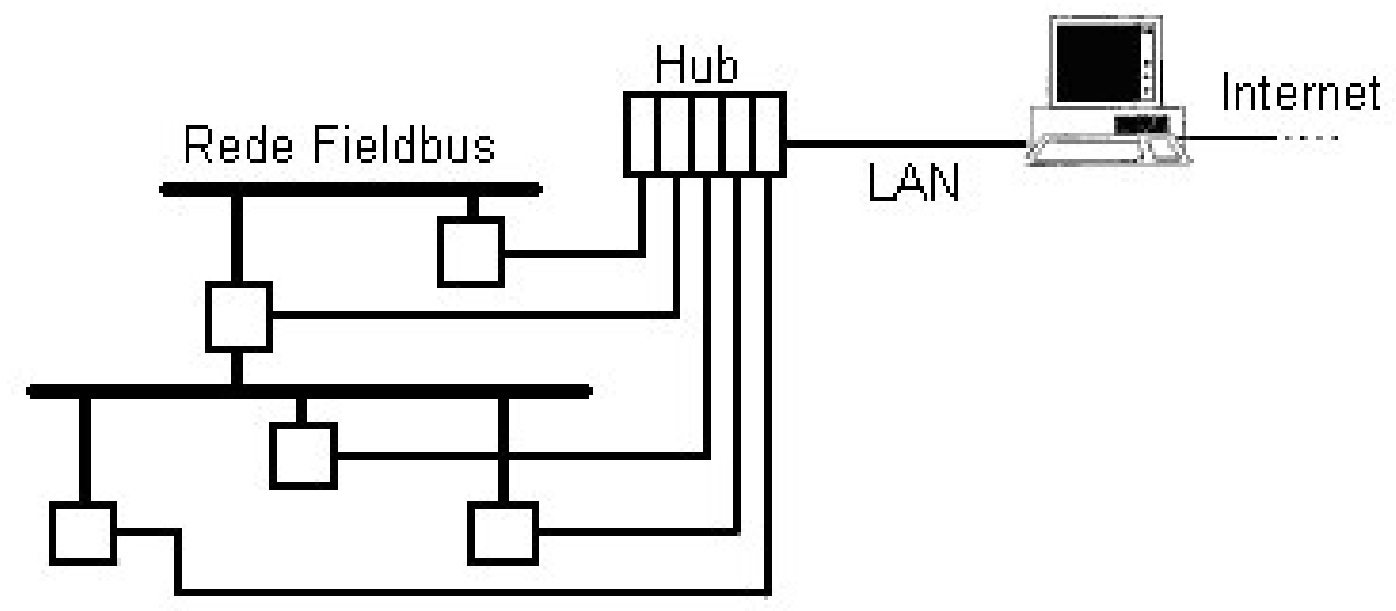

Figura 5. Agente SNMP implementado em cada dispositivo da rede de controle. Baseado em Knizak et al. (1997).

Uma outra solução é criar um proxy comum para os dispositivos da rede que seja capaz de prover a conversão do protocolo da rede de controle para a rede IP. Dessa forma seria aumentado o processamento de um dispositivo da rede capaz de traduzir as informações para o protocolo SNMP. Esse dispositivo trabalharia como uma espécie de gateway mapeando cada uma das informações da rede de controle para objetos SNMP.

Na Figura 6 é ilustrado o mapeamento do agente proxy SNMP. 


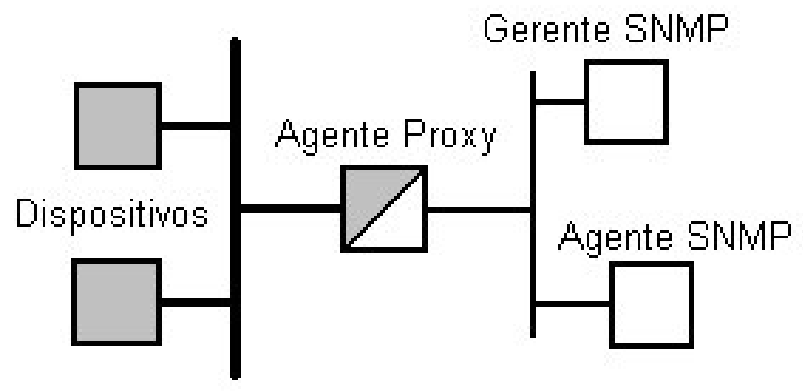

\begin{tabular}{|l|l|l|}
\hline \multicolumn{2}{c|}{} & \multicolumn{2}{|c|}{ Agente Proxy } \\
\hline Aplic. & Aplic. & SNMP \\
\hline Apres. \\
\hline Sessão \\
\hline Transp. \\
\hline Apres. & SNMP \\
\hline Sessão & SNMP \\
\hline Enlace \\
\hline Física \\
\hline Transp. & UDP \\
\hline Rede & IP \\
\hline Enlace & Enlace \\
\hline Física & Física \\
\\
\hline
\end{tabular}

Figura 6. Arquitetura do Agente Proxy SNMP. Baseado em Knizak et al. (1997). 


\section{TECNOLOGIA LONWORKS}

\subsection{INTRODUÇÃO}

Os sistemas de automação têm evoluído nos últimos anos devido à contínua busca em se criar uma nova geração de arquitetura de controle, com a intenção de obter uma maior flexibilidade e produtividade (MAHALIK; LEE, 2002). Antigamente, sistemas de controle inteiros eram projetados e desenvolvidos pelo mesmo fabricante, que detinha a responsabilidade sobre todo o sistema, e eram proprietários das arquiteturas de software e hardware utilizadas na aplicação da solução. Esse completo domínio de conhecimento pelo fabricante dificultava $O$ processo de troca entre fornecedor e cliente e a integração com outros sistemas. A arquitetura tradicionalmente utilizada nessas soluções era baseada em elementos centralizados. Cabos conectavam sensores e atuadores a um sub-painel, o qual era conectado através de um barramento mestre-escravo a um painel controlador central.

Porém, a tendência atual é que esse tipo de arquitetura venha a ser substituída por sistemas de controle descentralizados, onde dispositivos inteligentes interligados através de uma rede podem se comunicar utilizando protocolos apropriados e realizar o controle da rede (CHERMONT et al., 2005); (PEREIRA,2006). Na Figura 7 podemos observar essa mudança de paradigma.

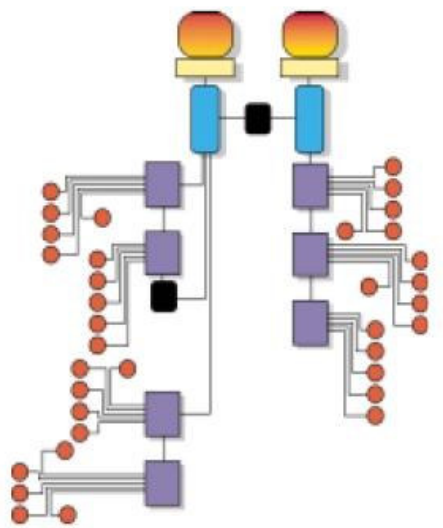

Solução Tradicional

Centralizada

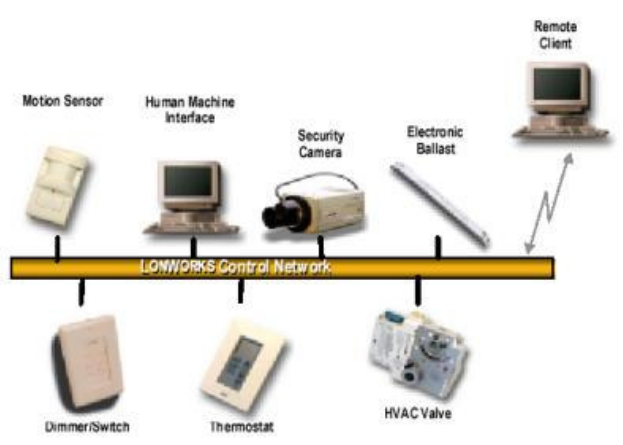

Novo Paradigma: Peer-to-Peer

Inteligência Distribuída

Figura 7. Tendência das soluções de automação. Extraído de Chermont et al. (2005). 
Seguindo essa tendência, a empresa Echelon criou a tecnologia LonWorks, que consiste em um certo número de dispositivos inteligentes, denominados nós, conectados entre si através de uma rede (Figura 8). Cada dispositivo é chamado de inteligente porque tem capacidade própria de processamento, podendo receber e enviar dados diretamente a outros dispositivos sem a necessidade de haver um controlador central para fazer o intermédio entre eles. A informação é transmitida através de pacotes de dados que podem ser recebidos por um ou mais nós (CANOVAS; CHERMONT, 2003); (ECHELON, 1999).

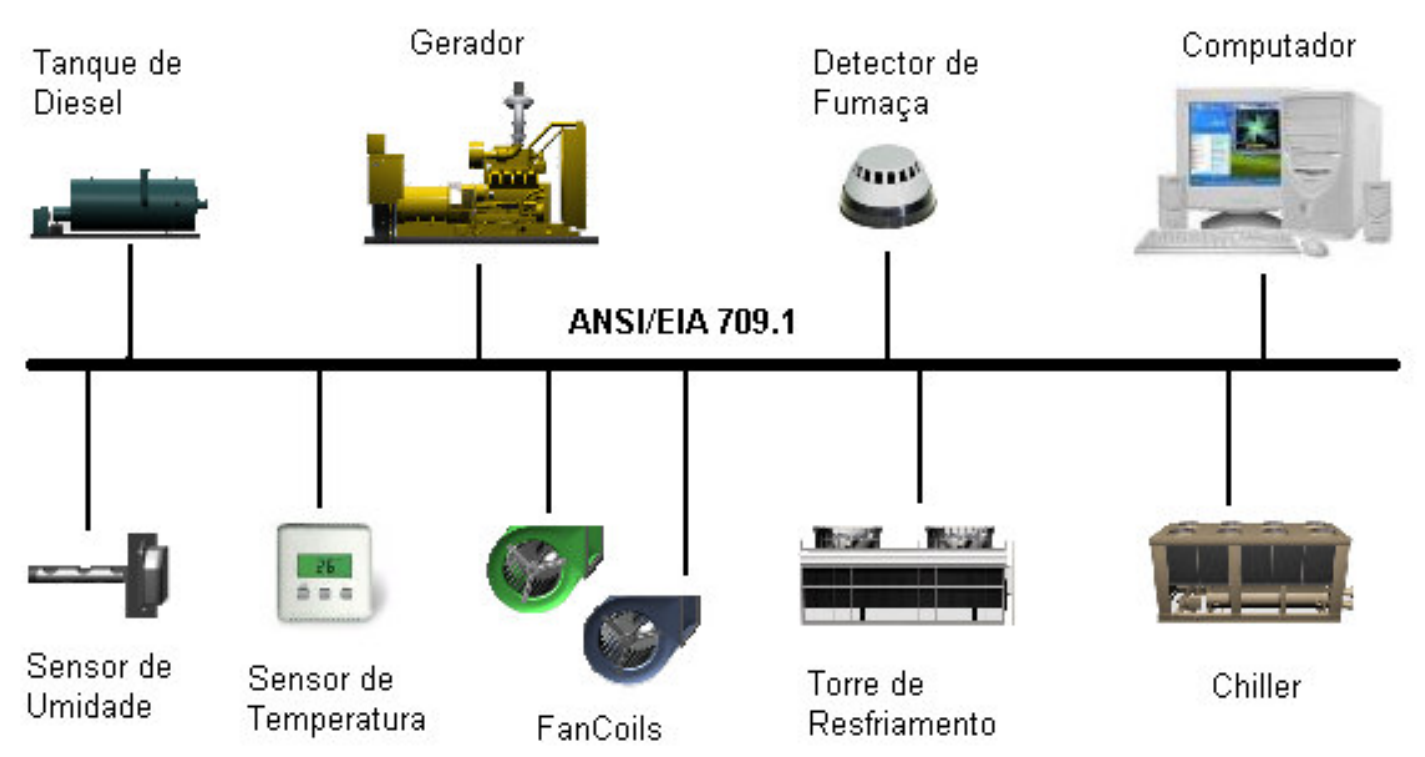

Figura 8. Rede de controle LonWorks.

Quando a empresa Echelon criou a tecnologia LonWorks ela havia decidido que essa tecnologia deveria primeiramente possuir um protocolo otimizado para redes de controle, porém, genérico o suficiente para trabalhar com diferentes tipos de controles que deveriam ser construídos. Mas deveria ter também um custo competitivo para o desenvolvimento desse protocolo nos dispositivos. E além de tudo, o protocolo deveria ser introduzido de uma maneira que não fosse possível realizar alterações de acordo com o fornecedor. O resultado foi o desenvolvimento de um sistema capaz de permitir que os fabricantes e instaladores se preocupem apenas com as aplicações tendo em mãos uma estrutura totalmente transparente e interoperável (ECHELON, 1999). 
Da mesma maneira que a Internet se tornou um padrão para as redes de dados, é um dos objetivos tornar os sistemas LonWorks os catalisadores da padronização das redes de controle. Pode-se considerar que isso está se tornando realidade ao observar que a tecnologia foi escolhida como padrão oficial europeu para sistemas de automação predial (ECHELON, 2007b).

A arquitetura de protocolos LonWorks é aberta e padronizada, o que permite que muitos fabricantes desenvolvam produtos compatíveis. Sua especificação pode ser facilmente encontrada e adquirida em IHS (2007). Além do protocolo padronizado estabeleceu-se um padrão, denominado padrão LonMark, que será visto mais adiante, que se tornou um poderoso instrumento para viabilizar a interoperabilidade de nós produzidos por empresas diferentes.

Um sistema aberto sob a abordagem LonWorks está ilustrado na Figura 9 onde se pode observar os três elementos-chave da tecnologia LonWorks: os dispositivos inteligentes que se comunicam entre si usando um protocolo sobre um ou mais meios de comunicação.

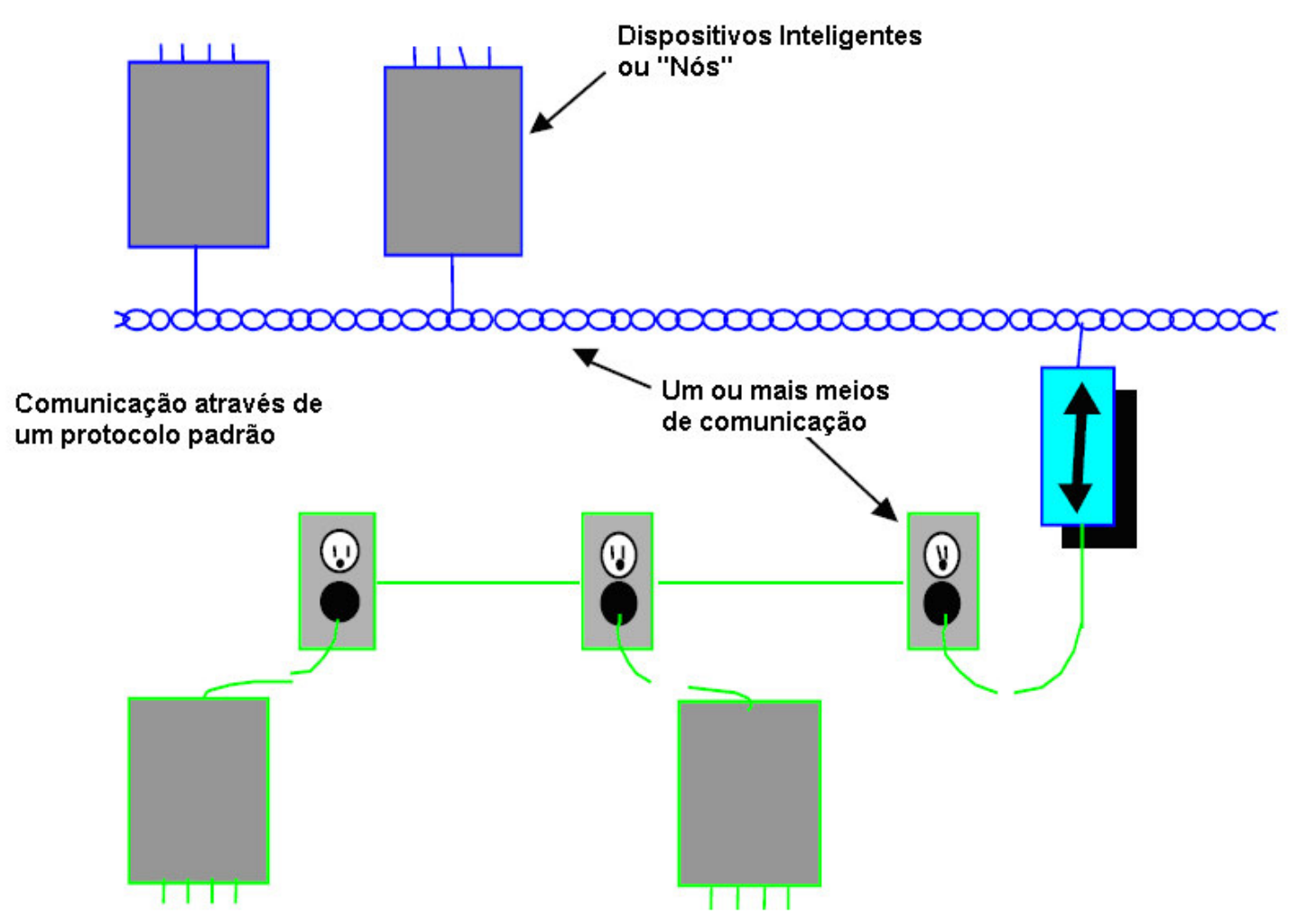

Figura 9. Elementos chaves da tecnologia LonWorks. Baseado em Echelon (1999). 
Os dispositivos incluem uma ou mais unidades de processamento que garantem a inteligência necessária para realizar a automação da rede. Eles implementam o protocolo de comunicação de maneira que possam ser conectados diretamente à rede, conforme a Figura 8. Dentro dos dispositivos existe um componente responsável pela conexão elétrica ao meio de transmissão, denominado transceiver. A tecnologia LonWorks padroniza diversos tipos de transceivers, um para cada meio de transmissão também padronizado, dentre eles 0 protocolo IP, que reserva a porta 1628 UDP para utilização de trocas de mensagens LonWorks como meio de comunicação (ECHELON, 2002a).

Os protocolos de comunicação definem o formato das mensagens que são trocadas entre os dispositivos, bem como as ações esperadas quando um nó envia uma mensagem para outro. A arquitetura de protocolos da tecnologia LonWorks normalmente toma forma de software embarcado ou firmware em cada dispositivo da rede. Como será visto mais adiante, um dos grandes trunfos da tecnologia LonWorks é o fato de o desenvolvedor de nós não precisar se preocupar com esta implementação, pois ela já pode ser encontrada pronta dentro dos processadores dos dispositivos (ECHELON, 1999).

O canal de comunicação é o meio físico por onde as informações trafegam. Os canais são classificados pelo seu tipo, o que determinará qual o transceiver que deve ser empregado, assim como afetará a velocidade de transmissão e as distâncias dos dispositivos na topologia de rede. Todos os nós conectados no mesmo canal devem possuir transceivers compatíveis entre si. Eles estão disponíveis para uma grande variedade de meios físicos, tais como:

- Par trançado;

- Rede elétrica;

- Rádio freqüência;

- Infra-vermelho;

- Fibra ótica;

- Cabo coaxial.

\subsection{PROTOCOLO LONTALK}

Especificado pela norma americana ANSI/EIA/CEA 709.1, o protocolo LonTalk, como também é conhecido, é a parte principal da tecnologia LonWorks. 
Conforme explica Echelon (1999), ele fornece um conjunto de serviços de comunicações que possibilitam aos programas aplicativos, que são executados nos dispositivos, enviarem e receberem mensagens sem a necessidade de conhecer a topologia da rede, nomes ou endereços dos outros nós. Ele oferece serviços de gerenciamento permitindo que ferramentas sejam capazes de interagir com os dispositivos e realizar reconfigurações dos endereços e dos parâmetros, realizar baixa de novas aplicações, reportar problemas da rede e iniciar/parar/reiniciar as aplicações disponíveis no dispositivo.

O protocolo LonWorks foi projetado para atender as necessidades dos sistemas de automação de maneira que fornecesse confiabilidade, performance e robustez, requisitos necessários para aplicações de controle. Dessa forma ele foi desenhado aderindo cada uma das camadas do modelo OSI de arquitetura de redes, padronizado pela International Organization for Standardization (ISO).

Toda comunicação se constitui na troca de um ou mais pacotes entre os dispositivos, onde esses pacotes podem variar de tamanho, porém são curtos o suficiente para minimizar o custo de implementação nos dispositivos e eles também contemplam cada um dos requisitos das sete camadas do modelo OSI.

Todos os dispositivos monitoram os pacotes que trafegam no meio de comunicação a fim de verificar quais estão endereçados a eles, que serão processados, verificando se o conteúdo é destinado à aplicação ou é um pacote de gerenciamento de rede. As informações relativas à aplicação são entregues e, se necessário, são geradas mensagens de recebimento, resposta ou autenticação para o emissor do pacote.

O protocolo LonTalk é independente do meio físico, permitindo que os dispositivos se comuniquem sobre qualquer meio físico de transporte. É denominado canal o meio físico de comunicação onde se encontra um grupo de dispositivos que utilizam o mesmo transceivers para se interligar.

\subsubsection{Endereçamento}

O endereçamento define como um dispositivo deverá ser capaz de localizar e direcionar uma mensagem para um outro dentro da rede. Um pacote pode ser direcionado para um ou vários dispositivos da rede e a tecnologia LonWorks suporta diferentes tipos de endereçamento, desde um simples direcionamento físico até um direcionamento que designa um grupo de dispositivos. 
Abaixo pode-se observar os diferentes tipos de endereçamento:

- Endereço Físico: todo o dispositivo LonWorks possui um identificador único de 48 bits chamado Neuron ID, que é análogo ao endereço Medium Access Control (MAC) das redes Ethernet. Ele geralmente é definido quando o dispositivo é construído e nunca mais é alterado. Não é possível que existam dois dispositivos com os mesmos Neuron ID;

- Endereço do Dispositivo: esse é um endereço lógico que facilita o mapeamento do dispositivo na rede. Ele é facilmente alterado através de mensagens de gerenciamento e é formado por uma estrutura de domínio, que pode ser descrito por até seis bytes, sub-rede (subnet), onde podem existir no máximo 255 dentro de um domínio e a identificação do nó (node). Dentro de uma subnet podem existir no máximo dois segmentos divididos por um repetidor totalizando 127 nós;

- Endereço de Grupo: um grupo é uma coleção lógica de dispositivos do mesmo domínio. Ela é uma maneira interessante de otimizar mensagens coletivas sem utilizar muita banda da rede. Podem existir no máximo 256 grupos em um domínio;

- Endereço de Transmissão (Broadcast): este endereçamento identifica todos os dispositivos de uma subnet ou de um domínio e é uma maneira eficiente de se comunicar com muitos dispositivos.

Todo o pacote LonWorks transmitido na rede contém o endereço de dispositivo daquele que está transmitindo (origem) e o endereço de destino, que pode ser um endereço físico, endereço do dispositivo, endereço de grupo ou endereço de transmissão.

\subsubsection{Mensagens}

O protocolo LonWorks oferece quatro tipos básicos de entrega de mensagem e também suporta o serviço de autenticação de mensagens, que permite ao receptor de uma mensagem identificar se o dispositivo que a enviou está 
autorizado a enviar a mensagem. A seguir apresentamos resumidamente os tipos disponíveis como apresentado em Canovas (2006):

- Acknowledged: fornece reconhecimento fim-a-fim. Quando um dispositivo envia uma mensagem ele aguarda a confirmação de cada destinatário;

- Repeated: a mensagem é enviada para um ou grupo de dispositivos várias vezes;

- Unacknowledged: a mensagem é enviada para um ou grupo de dispositivos e não é requisitado resposta;

- Request/Response: similar ao Acknowledged, porém a mensagem de confirmação é retornada juntamente com alguma resposta.

\subsubsection{Variáveis de rede}

Uma variável de rede (Network Variable ou NV) é uma estrutura de dados que simboliza uma variável do mundo real, como por exemplo, a temperatura de uma sala ou a pressão de um tanque. Essa estrutura é disponibilizada pelos dispositivos de maneira que possam ser compartilhadas na rede.

São essas informações que são trocadas entre os dispositivos para realizar a automação, dessa forma os programas que estão no interior dos dispositivos não precisam saber de onde vêm as informações de entradas para a lógica da aplicação e nem para onde devem ser enviadas os resultados do seu processamento, e sim quais estruturas representam as entradas do programa e quais outras representam as saídas. Essas estruturas representam os dois tipos de variáveis de rede:

- Variáveis de Entrada (Input Variables): são as estruturas de dados designadas para receber uma informação da rede. Eles representam a entrada de informação na lógica de um dispositivo;

- Variáveis de Saída (Output Variables): estruturas de dados responsáveis por fornecer as informações dos dispositivos. Por exemplo, um dispositivo sensor de temperatura irá disponibilizar na rede uma variável de rede que representa a temperatura registrada por ele.

A intenção ao criar esse tipo de estrutura no protocolo é permitir que se possa conectar uma variável de saída de um dispositivo a uma variável de entrada 
de um outro. Essa interconexão é chamada de binding e cria um cabo virtual entre os dois dispositivos fazendo com que os dois troquem informações de uma maneira transparente. A Figura 10 ilustra esse tipo de conexão.

É importante perceber que, para que as variáveis de entrada e de saída possam ser conectadas, ambas devem possuir formatos compatíveis. Assim, é impossível que uma variável de temperatura de saída se conecte a uma variável de pressão de entrada.

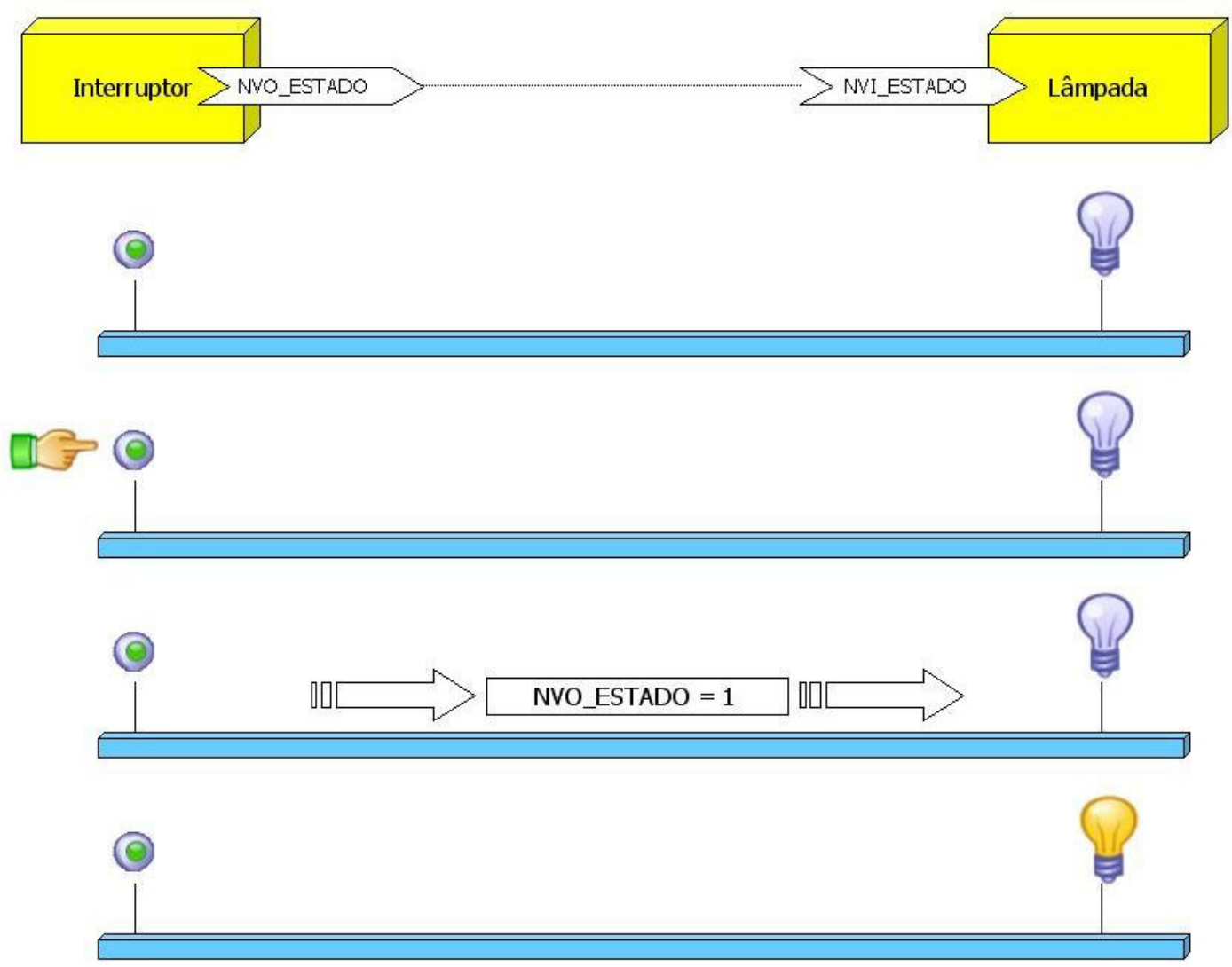

Figura 10. Conexão de variável de rede. Extraído de Canovas (2006).

\subsection{LONMARK}

Há uma diferença importante entre um conjunto de dispositivos interoperáveis e um sistema aberto. Como apresentado por Echelon (1999), é impossível existir um sistema aberto sem um conjunto de dispositivos interoperáveis, porém não necessariamente um conjunto de dispositivos interoperáveis representa um sistema aberto, podendo formar um sistema proprietário. 
Interoperabilidade significa que muitos dispositivos do mesmo ou de diferentes fabricantes possam se integrados em uma única rede de controle sem a necessidade de um dispositivo específico ou de uma programação especial (ECHELON, 1999).

Com a intenção de promover essa interoperabilidade, foi criada a LonMark, uma associação formada por fabricantes, integradores, usuários finais e outras empresas interessadas no avanço dessa tecnologia (ECHELON, 1999). Os objetivos principais da LonMark são especificar padrões de transceivers e meios físicos, entre os quais o mais usado comercialmente é o TP/FT-10 (Twisted Par Free Topology), que opera a 78 kbps, e definir padrões de estruturação e documentação de aplicações nos dispositivos.

Dentro dessa padronização encontram-se as Standard Network Variable Types (SNVT) que são as grandes mantenedoras da interoperabilidade entre os dispositivos. As SNVTs são padrões de estruturas de dados que caracterizam um tipo de variável de rede (NV). Porém a padronização LonMark define também padrões de variáveis de redes para a configuração de propriedades, as chamadas Standard Configuration Variable Types (SCPT), ou também as User-defined Configuration Variable Types (UNVT) que são padrões proprietários para configurar uma propriedade da aplicação.

Uma padronização interessante da LonMark são os Functional Profiles (Perfis Funcionais) que descrevem em detalhes a camada de interface da aplicação, incluindo as variáveis de rede, as propriedades de configuração e os procedimentos padrões ou de início que são necessários para alguns dispositivos LonMark específicos ou funções de controle comumente usadas. Apesar desse tipo de padronização não ser um produto, permite que diferentes fabricantes produzam produtos que possam ser facilmente trocados por de outros fabricantes, como é o caso dos controladores para Variable Air Volume (VAV).

Um dado dispositivo LonWorks pode possuir diferentes perfis funcionais, 0 que garante diferentes funcionalidades padronizadas a ele. Quando um dado dispositivo implementa diferentes perfis funcionais, é obrigatório que seja implementado o perfil funcional "Node Object", que irá gerenciar os outros diferentes perfis dentro do dispositivo. Na Figura 11 pode-se ver a estrutura de um dispositivo com diferentes perfis funcionais implementados. 


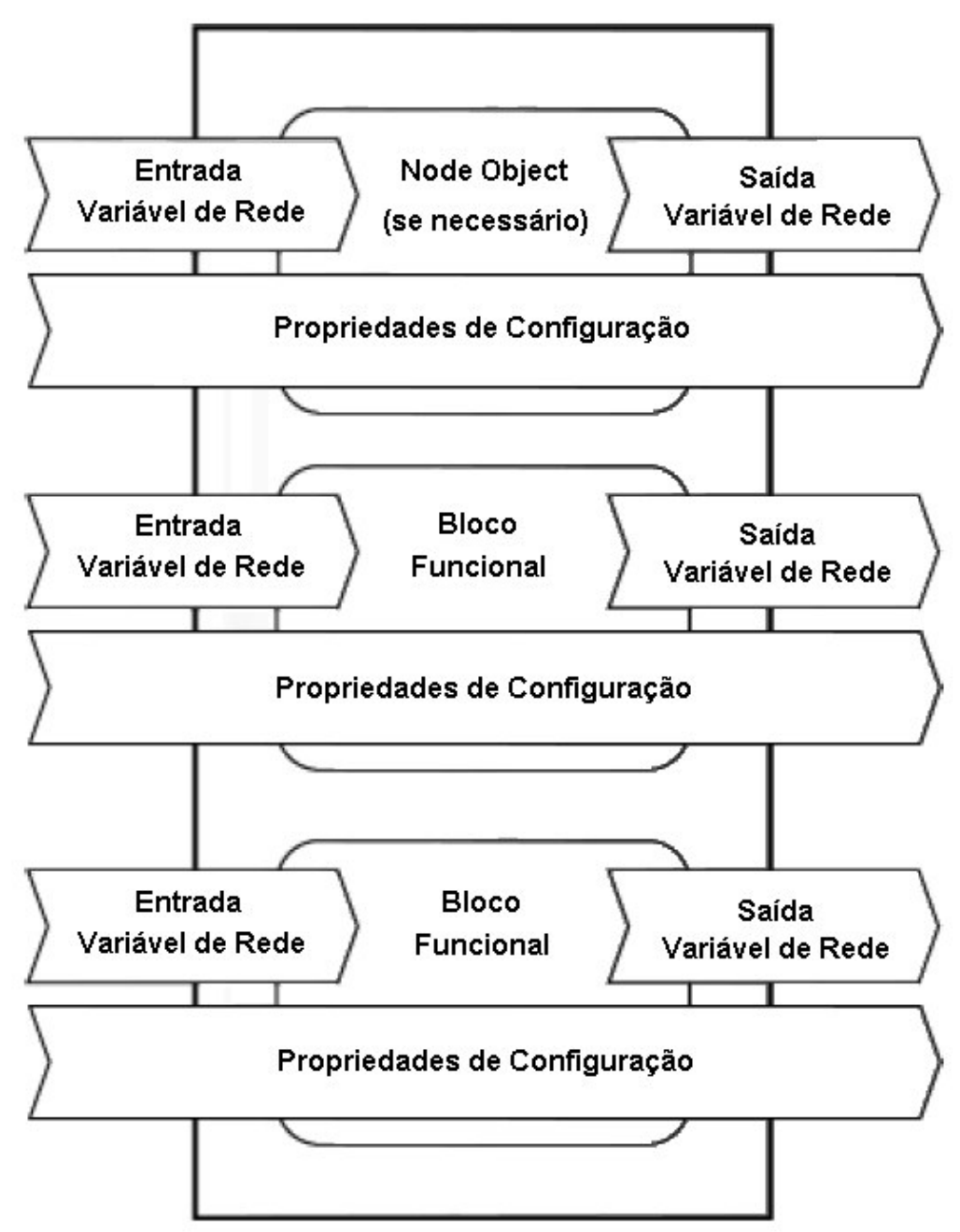

Figura 11. Dispositivo com diferentes perfis funcionais. Baseado em Echelon (2002b).

De maneira a identificar os dispositivos certificados pela LonMark foi criado um selo (Figura 12) que caracteriza que o dispositivo é interoperável e permite que o consumidor possa automaticamente identificar a interoperabilidade. Juntamente com o logotipo é apresentado o número da versão utilizada na certificação. 


\section{腺}

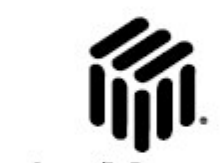

LONMARK"3,3

\section{陑}

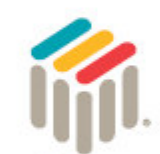

LONMARK'3.3

\section{流(}

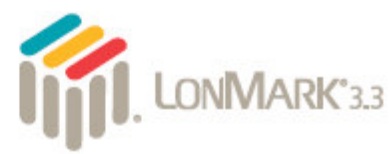

Figura 12. Logotipos LonMark. Extraído de Echelon (2002b).

\subsection{NEURON CHIP}

Com a intenção de atingir os objetivos econômicos e de padronização, a Echelon criou o Neuron Chip, que é um chip que contém 3 processadores que implementam as camadas de 1 a 6 do protocolo LonTalk. Um processador é encarregado de se comunicar com a rede implementando as camadas 1 e 2, enquanto o segundo faz interligação entre o primeiro e o terceiro, implementando as camadas de 3 a 6 . $O$ terceiro e último processador fica dedicado ao processamento da camada 7 de aplicação. Isso permite que se tomem vantagens das funções do Neuron Chip utilizando-o como processador da aplicação e não só como interface de rede (CANOVAS; CHERMONT, 2003); (ECHELON, 1999).

Pode-se observar que o encapsulamento do protocolo LonTalk no Neuron Chip facilita o desenvolvimento de novos dispositivos, pois permite que os fabricantes se preocupem apenas em desenvolver as aplicações que deverão estar contidas ou na memória interna do chip ou numa memória externa auxiliar.

O desenvolvimento de aplicações para dispositivos LonWorks é feito através da linguagem Neuron $C$, que é muito similar a linguagem $C$.

$\mathrm{Na}$ Figura 13, pode-se observar a estrutura completa de um dispositivo LonWorks, onde se encontra o Neuron Chip com os seus três processadores internos, o transceiver responsável por conectar o dispositivo ao meio físico, a memória externa opcional, a alimentação e também as portas de entradas e saídas 
elétricas disponíveis no Neuron Chip para serem integradas com outros dispositivos elétricos e constituírem as funções do dispositivo.

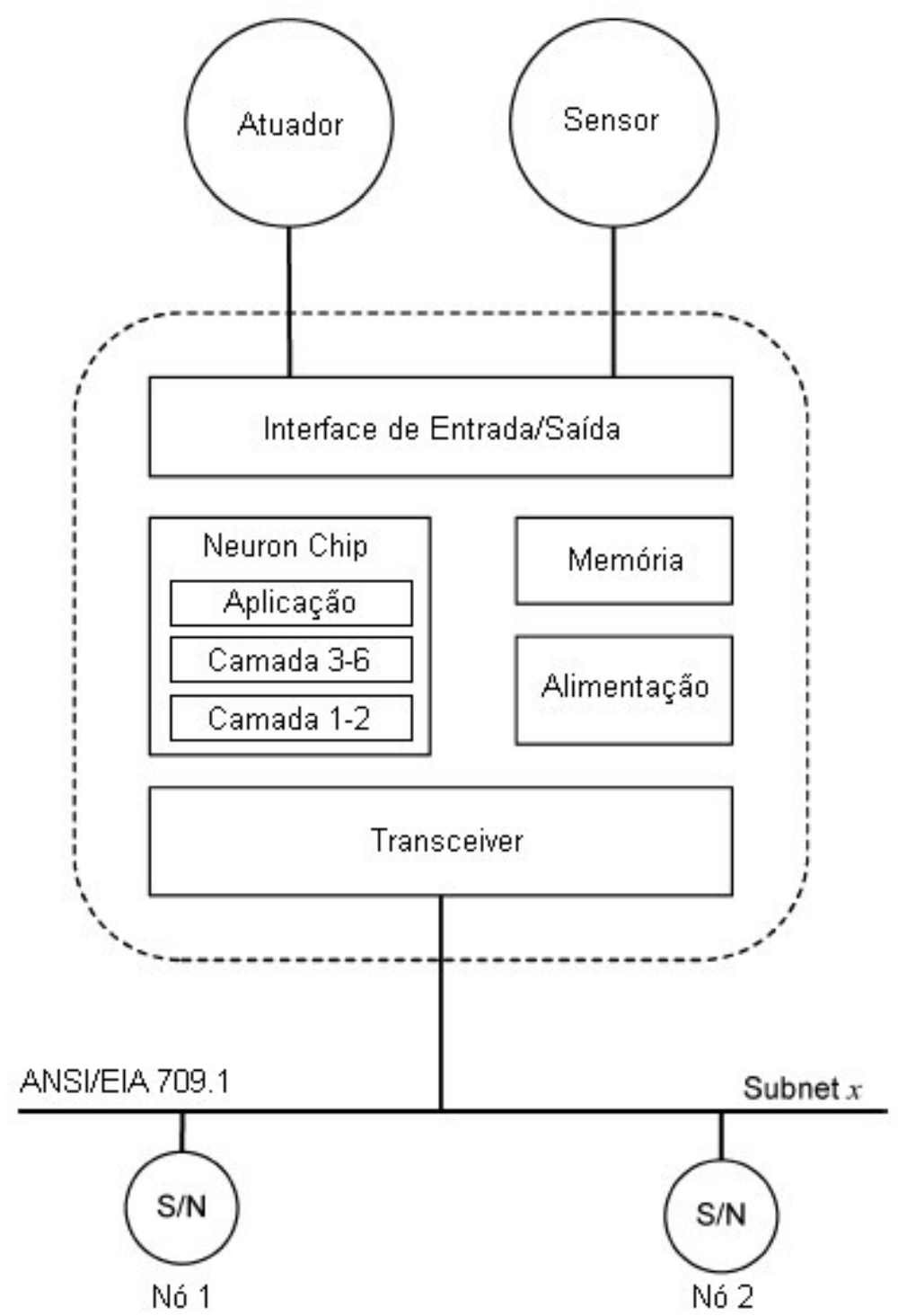

Figura 13. Dispositivo LonWorks. Baseado em Kastner et al. (2005).

\subsection{GERENCIAMENTO}

O protocolo LonTalk oferece o serviço de gerenciamento de rede possibilitando a instalação e configuração dos nós, o download de programas e o diagnóstico da rede. As mensagens são dividas em dois tipos, mensagens de gerenciamento e mensagem de diagnóstico. Elas são do tipo Request/Response, onde existe uma resposta associada a cada requisição. $\mathrm{Na}$ Tabela 1 podemos 
observar as mensagens de gerenciamento disponíveis assim como os seus respectivos códigos.

Tabela 1 - Mensagens de gerenciamento de rede.

\begin{tabular}{|c|c|c|c|}
\hline Mensagem & $\begin{array}{l}\text { Código da } \\
\text { Requisição }\end{array}$ & $\begin{array}{c}\text { Resposta de } \\
\text { Sucesso }\end{array}$ & $\begin{array}{c}\text { Resposta de } \\
\text { Falha }\end{array}$ \\
\hline Query ID & $0 \times 61$ & $0 \times 21$ & $0 \times 01$ \\
\hline Respond to Query & $0 \times 62$ & $0 \times 22$ & $0 \times 02$ \\
\hline Update Domain & $0 \times 63$ & $0 \times 23$ & $0 \times 03$ \\
\hline Leave Domain & $0 \times 64$ & $0 \times 24$ & $0 \times 04$ \\
\hline Update Key & $0 \times 65$ & $0 \times 25$ & $0 \times 05$ \\
\hline Update Address & $0 \times 66$ & $0 \times 26$ & $0 \times 06$ \\
\hline Query Address & $0 \times 67$ & $0 \times 27$ & $0 \times 07$ \\
\hline Query Net Variable Config & $0 \times 68$ & $0 \times 28$ & $0 \times 08$ \\
\hline Update Group Address Data & $0 \times 69$ & $0 \times 29$ & $0 \times 09$ \\
\hline Query Domain & $0 \times 6 \mathrm{~A}$ & $0 \times 2 A$ & $0 \times 0 A$ \\
\hline Update Net Variable Config & $0 \times 6 \mathrm{~B}$ & $0 \times 2 B$ & $0 \times 0 B$ \\
\hline Set Node Mode & $0 \times 6 \mathrm{C}$ & $0 \times 2 \mathrm{C}$ & $0 \times 0 \mathrm{C}$ \\
\hline Read Memory & $0 \times 6 \mathrm{D}$ & $0 \times 2 D$ & $0 \times 0 D$ \\
\hline Write Memory & $0 \times 6 E$ & $0 \times 2 E$ & $0 \times 0 E$ \\
\hline Checksum Recalculate & $0 \times 6 \mathrm{~F}$ & $0 \times 2 F$ & $0 \times 0 F$ \\
\hline Wink & $0 \times 70$ & $0 \times 30$ & $0 \times 10$ \\
\hline Memory Refresh & $0 \times 71$ & $0 \times 31$ & $0 \times 11$ \\
\hline Query SNVT & $0 \times 72$ & $0 \times 32$ & $0 \times 12$ \\
\hline Network Variable Fetch & $0 \times 73$ & $0 \times 33$ & $0 \times 13$ \\
\hline Device Escape Code & $0 \times 7 D$ & $0 \times 3 D$ & $0 \times 1 D$ \\
\hline
\end{tabular}

Fonte: Toshiba (1995).

Foge do escopo desse trabalho descrever cada uma das mensagens de gerenciamento. Para validar o desenvolvimento do agente proxy SNMP, concentrarse-á nas mensagens de diagnóstico, onde será possível avaliar o estado de cada um dos dispositivos da rede. A Tabela 2 apresenta as mensagens de diagnóstico disponíveis assim como os seus respectivos códigos. 
Tabela 2 - Mensagens de diagnóstico de rede.

\begin{tabular}{lccc}
\hline \multicolumn{1}{c}{ Mensagem } & $\begin{array}{c}\text { Código da } \\
\text { Requisição }\end{array}$ & $\begin{array}{c}\text { Resposta de } \\
\text { Sucesso }\end{array}$ & Resposta de Falha \\
\hline Query Status & $0 \times 51$ & $0 \times 31$ & $0 \times 11$ \\
Proxy Command & $0 \times 52$ & $0 \times 32$ & $0 \times 12$ \\
Clear Status & $0 \times 53$ & $0 \times 33$ & $0 \times 13$ \\
Query XCVR Status & $0 \times 54$ & $0 \times 34$ & $0 \times 14$ \\
\hline
\end{tabular}

Fonte: Toshiba (1995).

A mensagem Query Status recupera as informações de estatísticas de erro da rede, a causa da última reinicialização, o estado do dispositivo e o último erro de execução. Dentro da mensagem de resposta estão incluídos os seguintes itens:

- Erros de Transmissão: números de erros de Cyclic Redundancy Check (CRC) detectados durante a recepção de pacotes. Eles podem ser causados por colisões ou ruídos na rede;

- Tempo Máximo de Transação: número de vezes em que se excedeu o tempo máximo de espera por uma resposta após o reenvio da mensagem o número de vezes previamente configurado;

- Erros na Recepção de Transações Completas: número de vezes, em que os pacotes que estavam chegando, foram desconsiderados, por não haver espaço no banco de dados de transações;

- Mensagens Perdidas: número de vezes, em que pacotes que estavam chegando, foram desconsiderados, por não haver espaço de armazenamento no buffer da aplicação;

- Mensagens com Falha: número de vezes, em que os pacotes que estavam chegando, foram desconsiderados, por não haver espaço de armazenamento no buffer da rede;

- Causa da Última Reinicialização: indica o motivo do último reinício do nó. Podem ser os seguintes motivos:

- Power-up reset (0x01);

- External reset (0x02);

- Watchdog reset (0x0C);

- Software reset (0x14);

- Cleared (0x00). 
- Estado do Nó: Contém o estado atual do nó de acordo com as seguintes definições:

- Não configurado e sem aplicação (0x03);

- Não configurado e com aplicação (0x02);

- Configurado, hard off-line (0x06);

- Configurado, soft off-line $(0 \times 0 \mathrm{C})$;

- Configurado, bypass off-line (0x8C);

- Configurado, on-line (0x04).

- Versão do Firmware: número da versão do firmware sendo executado no nó;

- Último Erro: indica a causa do último erro ocorrido;

- Modelo do Neuron Chip: contém o número do modelo do Neuron Chip, "0x00" para o modelo 3150 e "0x08" para o 3120.

Esta mensagem de diagnóstico foi mapeada em objetos MIBs de maneira que essas informações possam estar disponíveis ao gerente SNMP. As outras mensagens de gerenciamento e diagnóstico não foram implementadas neste trabalho, ficando para possíveis melhorias e continuação. 


\section{AGENTE PROXY SNMP}

\subsection{ARQUITETURA DA SOLUÇÃO}

A solução adotada para a implementação do agente proxy SNMP prevê a concentração do gerenciamento dos dispositivos da rede LonWorks em um único agente. Esse agente implementa um conjunto de objetos MIB descritos no item 4.3 deste trabalho, capazes de traduzir uma série de informações de diagnóstico de um nó da rede para um gerente SNMP.

A Figura 14 apresenta a arquitetura da solução adotada onde se pode observar a estrutura de comunicação do agente com a rede LonWorks e com o gerente SNMP.

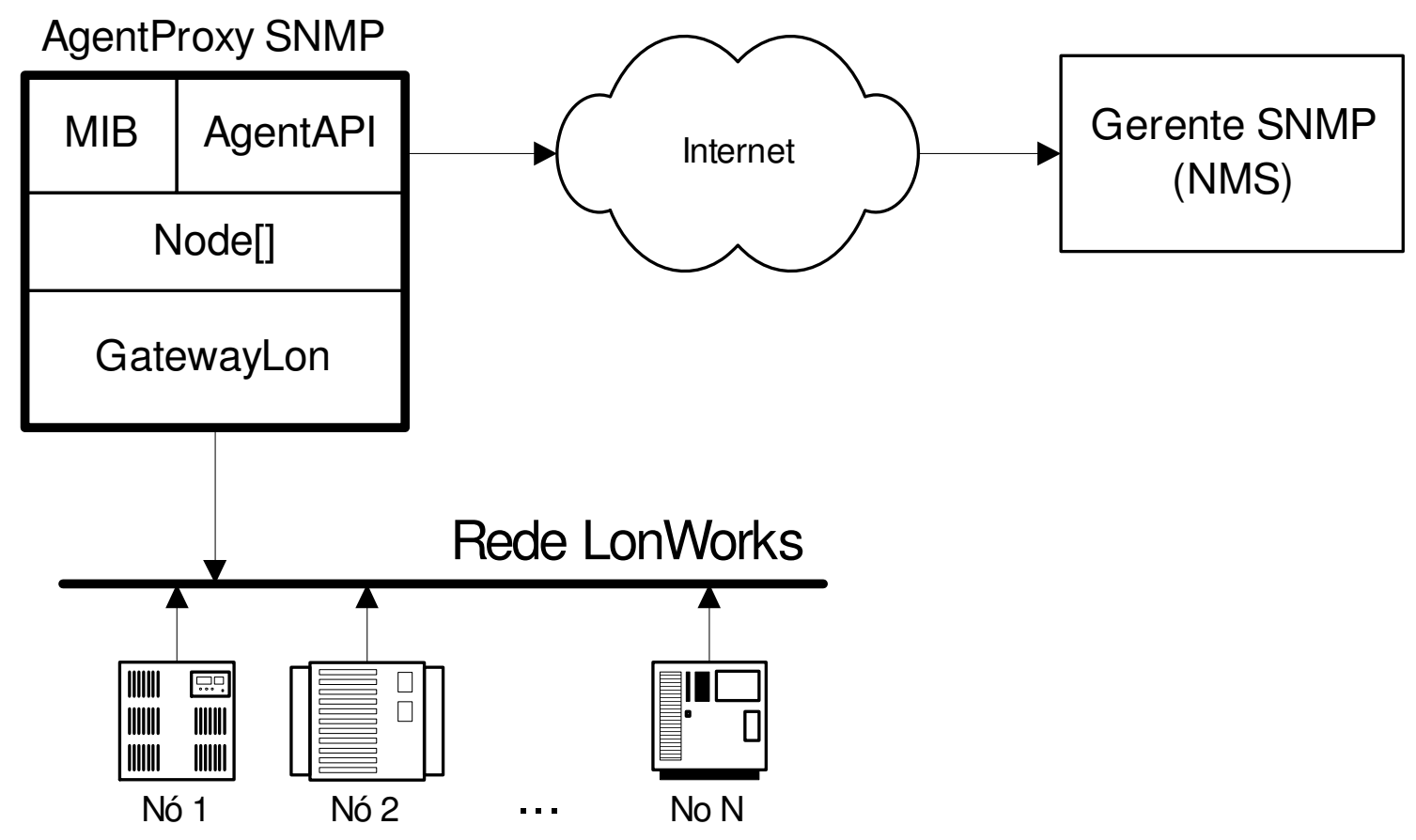

Figura 14. Arquitetura da solução proposta.

A estrutura interna do agente proxy é dividida em quatro partes:

- AgentAPI: API responsável pela implementação da comunicação SNMP;

- MIB: conjunto de informações que representam características do dispositivo que estão disponíveis para o gerente SNMP; 
- Node[]: vetor de dispositivos que representa cada um dos nós a serem gerenciados;

- GatewayLon: responsável por realizar a comunicação com a rede Lonworks.

\subsection{AGENTAPI}

Para a implementação do agente proxy SNMP foi utilizada uma API desenvolvida pelo Professor Rui Pedro Sanches de Castro Lopes do Instituto Politécnico de Bragança, Portugal (LOPES; OLIVEIRA, 2003). A API foi disponibilizada em forma de um package Java, chamado AgentAPI, regido pela licença GNU's Not Unix (GNU). Ela implementa a modelagem do agente SNMP e suas tarefas comuns, facilitando o processo de desenvolvimento.

Dessa forma, as estruturas de dados compatíveis com o SNMP já estão implementadas nessa API, assim como as mensagens e as rotinas de tratamento de resposta. Isso facilitou o trabalho de desenvolvimento do agente SNMP, permitindose focar no tratamento dos dados da rede LonWorks e na sua integração com o agente.

Não fez parte do escopo desse trabalho detalhar a estrutura da API, sendo possível encontrar informações mais detalhadas em Lopes (2003).

\subsection{MIB}

O módulo que representa a MIB, desenvolvida nesse trabalho, foi denominada LON-NODE-MIB e foi colocado sob o ramo experimental, por ser o ramo dedicado para MIBs em fase de testes. Dentro do ramo experimental foi criado - OID lonworks, com a proposta de agrupar todas as MIBs relativas a tecnologia LonWorks. Além disso, foi criada também a OID nodes, que irá agrupar todas as informações relativas aos diferentes dispositivos que deverão ser instanciados na MIB. Assim, tem-se a seguinte representação completa da MIB criada:

.iso.org.dod.internet.experimental.lonworks.nodes (.1.3.6.1.3.1.1)

De modo a organizar as informações disponíveis na MIB, ela foi dividida em três conjuntos de objetos: a primeira subdivisão traz informações relativas ao 
software agente, a segunda é uma tabela indexada por identificador do nó que traz informações gerais do dispositivo, e a última subdivisão é uma outra tabela com o mesmo índice e com as informações de diagnóstico detalhadas no item 3.5 deste documento.

\subsubsection{Informações do agente}

Essa parte da MIB é dedicada a objetos que fornecem informações sobre o proxy agente implementado e eles estão disponíveis somente para leitura. A Tabela 3 detalha cada um dos objetos disponíveis.

Tabela 3 - Informações sobre o proxy agente.

\begin{tabular}{|c|c|}
\hline Objeto & Descrição \\
\hline nodeCount (1) & $\begin{array}{l}\text { Apresenta o número total de dispositivos monitorados } \\
\text { pelo agente. }\end{array}$ \\
\hline nodeAgentDescr (2) & $\begin{array}{l}\text { Retorna a descrição do agente SNMP, o nome do } \\
\text { sistema operacional, a versão do agente, a } \\
\text { plataforma de desenvolvimento, etc. }\end{array}$ \\
\hline nodeAgentContact (3) & Informa o contato do desenvolvedor do agente. \\
\hline nodeAgentLocation (4) & Informa a localização do agente. \\
\hline nodeAgentTime (5) & Informa quanto tempo o agente esta em execução. \\
\hline
\end{tabular}

\subsubsection{Informações de configuração do nó}

Essa parte da MIB define uma tabela com a descrição dos dispositivos monitorados pelo agente. A Figura 15 apresenta a estrutura desse objeto tabular apresentada através da captura de tela do software AdventNet MIB Editor. 


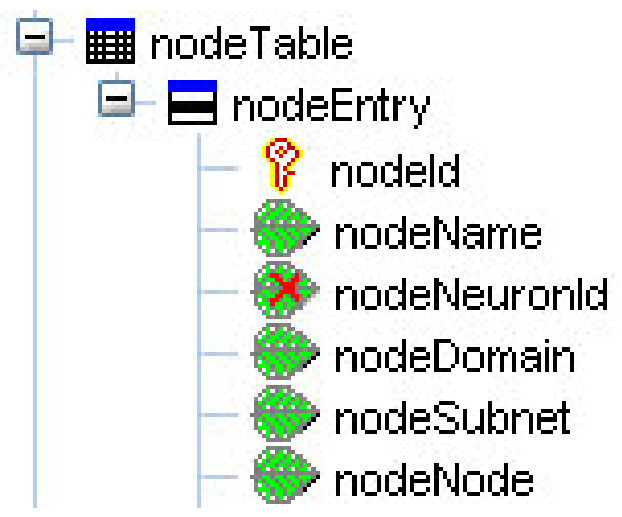

Figura 15. Objeto NodeTable de informações dos dispositivos.

A Tabela 4 apresenta a descrição de cada um dos objetos.

Tabela 4 - Informações gerais sobre os dispositivos.

\begin{tabular}{ll}
\hline \multicolumn{1}{c}{ Objeto } & \multicolumn{1}{c}{ Descrição } \\
\hline nodeld (1) & Identificador único do nó na tabela. \\
nodeName (2) & Nome descritivo do dispositivo. \\
nodeNeuronld (3) & Neuronld do dispositivo; campo de somente leitura. \\
nodeDomain (4) & Domínio a que pertence o dispositivo. \\
nodeSubnet (5) & Subnet a que pertence o dispositivo. \\
nodeNode (6) & Número do nó na rede. \\
\hline
\end{tabular}

\subsubsection{Informações de diagnóstico do nó}

Este objeto tabular traz as informações de diagnóstico descritas no item 3.5 deste trabalho. A Figura 16 apresenta a estrutura desse objeto tabular apresentada através da captura de tela do software AdventNet MIB Editor. 


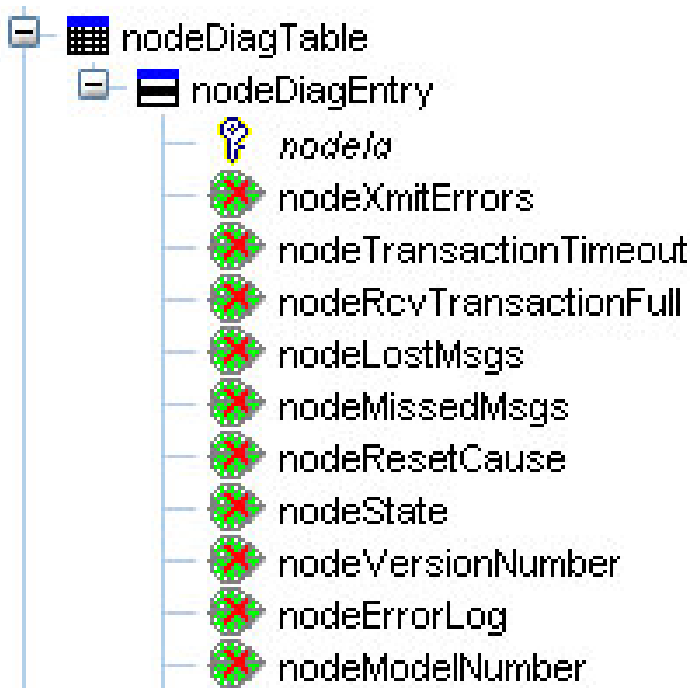

Figura 16. Objeto NodeDiagTable de informações de diagnóstico do dispositivo.

Todos os objetos desta tabela são somente leitura, pois retratam a situação atual do dispositivo. A Tabela 5 descreve resumidamente cada um dos objetos dessa tabela.

Tabela 5 - Informações de diagnóstico dos dispositivos.

\begin{tabular}{ll}
\hline \multicolumn{1}{c}{ Objeto } & \multicolumn{1}{c}{ Descrição } \\
\hline nodeXmitErrors (1) & Número de erros de transmissão. \\
nodeTransactionTimeout (2) & Número de timeouts ocorridos. \\
nodeRcvTransactionFull (3) & $\begin{array}{l}\text { Número de erros na recepção de transações } \\
\text { completas. }\end{array}$ \\
nodeLostMsgs (4) & Número de mensagens perdidas. \\
nodeMissedMsgs(5) & Número de mensagens com falhas. \\
nodeResetCause (6) & Causa da última reinicialização. \\
nodeState (7) & Estado do nó. \\
nodeVersionNumber (8) & Número da versão do firmware. \\
nodeErrorLog (9) & Último erro ocorrido. \\
nodeModelNumber (10) & Modelo do NeuronChip. \\
\hline
\end{tabular}




\subsection{NODE[]}

Com a intenção de isolar as requisições do gerente SNMP das consultas a uma rede LonWorks, foi criado um vetor de objetos, capaz de representar todos os dispositivos que deverão ser monitorados pelo agente. Cada um dos dispositivos é mapeado em um objeto independente, que possui as informações específicas de cada nó e é responsável por consultar periodicamente o estado do mesmo na rede LonWorks. Essa estrutura independente impede que sucessivas requisições do gerente SNMP acarretem uma sobrecarga de mensagens na rede LonWorks.

A classe Node desenvolvida possui parâmetros que representam cada um dos objetos da MIB LON-NODE-NIB criada neste trabalho. A Figura 17 apresenta a estrutura da classe Node com os seus respectivos parâmetros; ela implementa a classe Runnable do Java, que possibilita a criação de um processamento paralelo para a captura de informações na rede LonWorks, sem interromper a execução do agente. Isso é realizado em intervalos de tempo definidos pelo atributo sampling do objeto, que inicia com o valor padrão de dois segundos de amostragem.

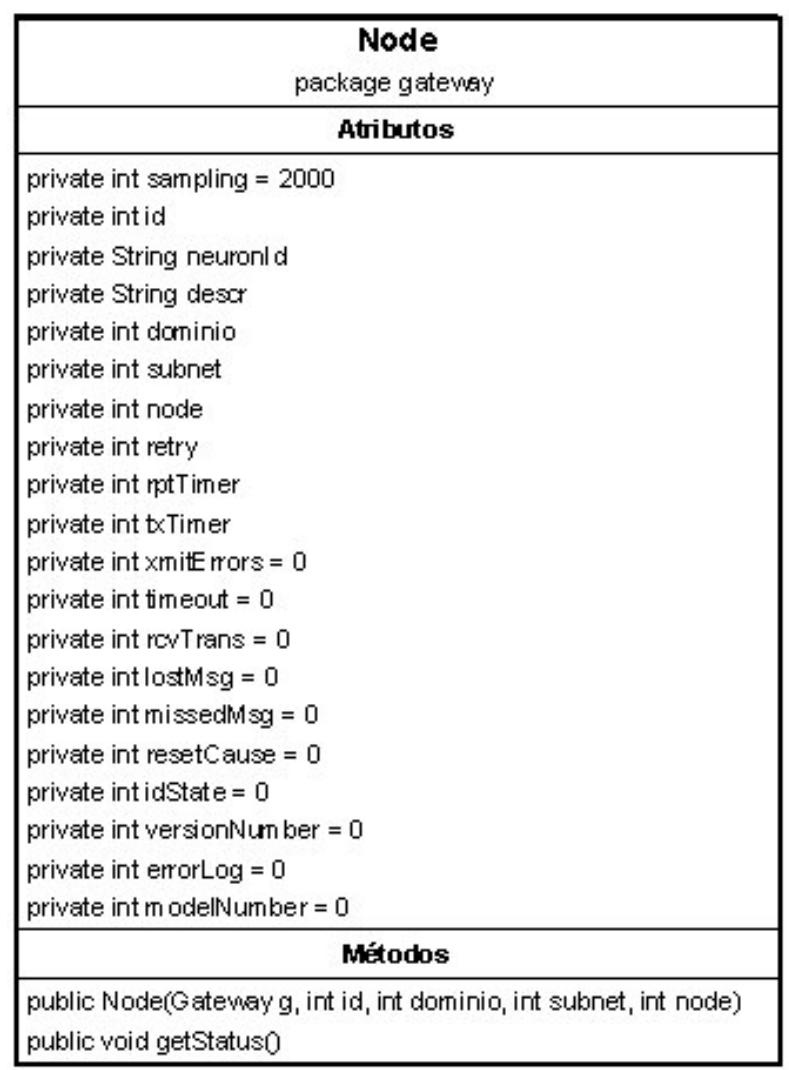

Figura 17. Classe Node criada para representar um dispositivo. 
A integração dos atributos da classe Node em objetos MIB foi realizada através de classes que representam cada um dos objetos da MIB LON-NODE-MIB. Elas são instanciadas e armazenadas em uma estrutura da AgentAPI, capaz de relacioná-las com os OIDs dos objetos da MIB, de maneira que são chamadas quando recebem um requisição do gerente SNMP e são capazes de retornar o valor para o agente proxy que a transforma numa resposta SNMP. A Tabela 6 apresenta a lista de classes criadas para mapear a MIB LON-NODE-MIB.

Tabela 6 - Lista de classes que implementam os objetos da MIB LON-NODE MIB.

\begin{tabular}{|c|c|}
\hline Classes & Descrição \\
\hline NodeAgentContact & $\begin{array}{l}\text { Implementa o objeto nodeAgentContact, retornando } \\
\text { informações de contato do desenvolvedor do agente. }\end{array}$ \\
\hline NodeAgentDescr & $\begin{array}{l}\text { Implementa o objeto nodeAgentDescr retornando a } \\
\text { descrição do agente SNMP, o nome do sistema } \\
\text { operacional, a versão do agente, a plataforma de } \\
\text { desenvolvimento, etc. }\end{array}$ \\
\hline NodeAgentLocation & $\begin{array}{l}\text { Implementa o objeto nodeAgentLocation, retornando } \\
\text { a localização do agente. }\end{array}$ \\
\hline NodeAgentTime & $\begin{array}{l}\text { Implementa o objeto nodeAgentTime, retornando a } \\
\text { quantidade de tempo que o agente esta em } \\
\text { execução. }\end{array}$ \\
\hline NodeDiagnosticTable & $\begin{array}{l}\text { Implementa o objeto tabular nodeDiagnosticTable, } \\
\text { sendo capaz de retornar qualquer um dos objetos } \\
\text { disponíveis na tabela e relacionar cada OID com um } \\
\text { dispositivo específico. }\end{array}$ \\
\hline NodeTable & $\begin{array}{l}\text { Implementa o objeto tabular nodeTable, retornando } \\
\text { os objetos que descrevem o dispositivo. É capaz de } \\
\text { relacionar um OID com as informações de um } \\
\text { dispositivo específico. }\end{array}$ \\
\hline
\end{tabular}

A Figura 18 apresenta a relação dessas classes e como o agente foi implementado. 


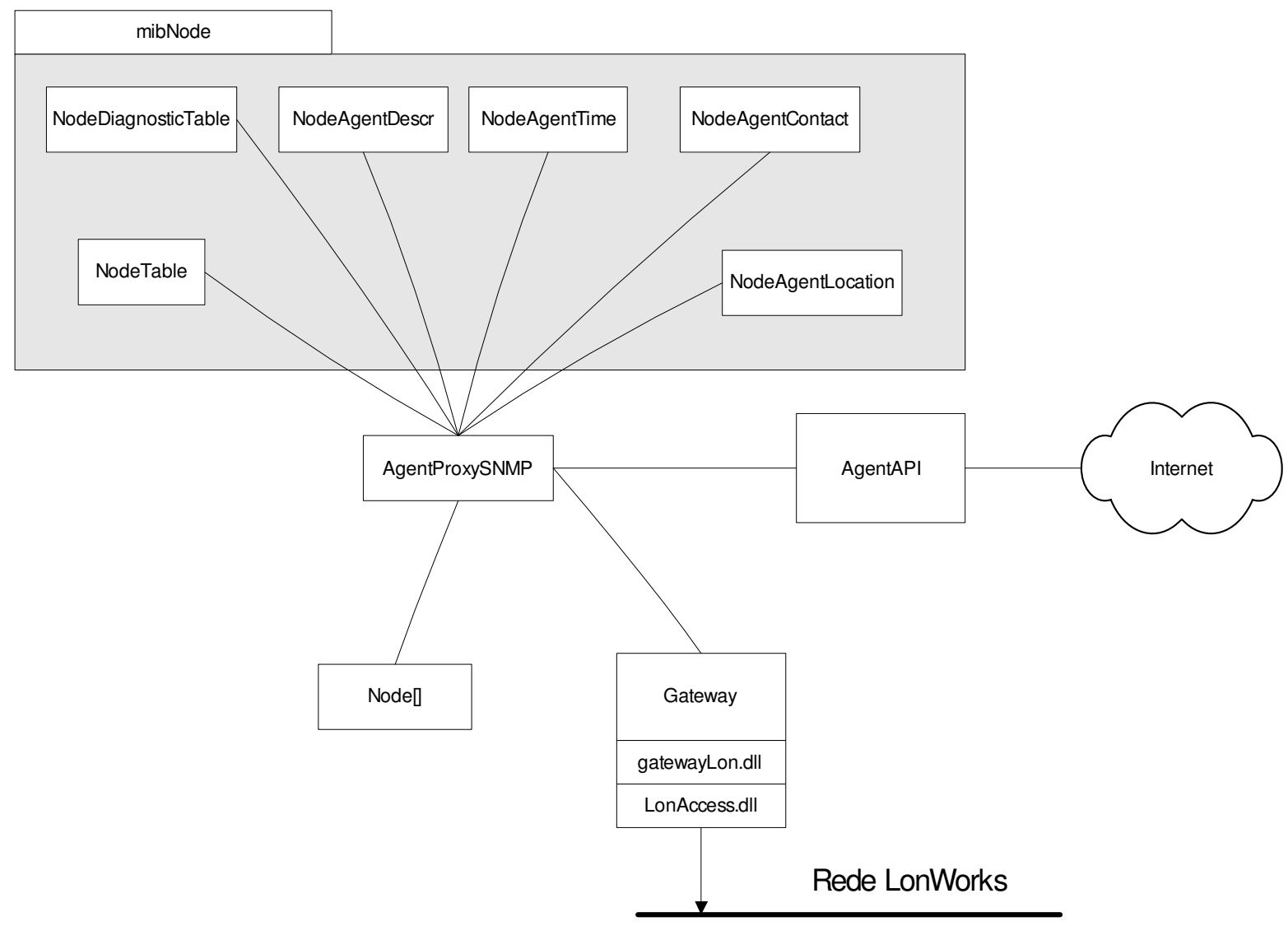

Figura 18. Estrutura interna do agente proxy SNMP.

\subsection{GATEWAY LON}

Toda a comunicação com a rede LonWorks foi encapsulada em uma classe gateway, em Java que realiza o acesso as rotinas disponíveis em uma Dynamic Link Library $(D L L)$, denominada "LonAccess.dll", desenvolvida no trabalho de Canovas; Chermont; Cugnasca (2005) para comunicação com a rede LonWorks. Dessa maneira é possível utilizar os mesmos métodos disponíveis na $D L L$ através da classe gateway de uma maneira transparente.

Para atender as necessidades desse trabalho foi necessário ampliar as funções disponíveis na DLL “LonAccess.dll", implementando uma mensagem diagnóstico do protocolo LonTalk, que permite capturar uma série de informações sobre o estado do dispositivo através de uma única requisição. Informações mais detalhadas sobre essa mensagem se encontram no item 3.5 deste trabalho.

A seguir detalha-se cada uma das funções disponíveis na biblioteca:

- int lon_init(String device_name): é necessário chamar esse método antes de qualquer operação com a rede LonWorks pois 
ele é responsável por inicializar o dispositivo de acesso à rede. Deve ser enviado como parâmetro o nome do dispositivo e a função retornará o valor "0" em caso de sucesso;

- void lon_close () : finaliza a utilização do dispositivo de acesso à rede LonWorks o liberando para outras aplicações;

- void lon_set_dest_addr_sn(int domainIndex, int subnet, int node, int retry, int rptTimer, int txTimer): este método configura o endereço de destino através do endereçamento Subnet/Node, informando o domínio, subnet e node do dispositivo. O parâmetro retry representa o número de tentativas que devem ser feitas para contactar o dispositivo de destino, caso houver erro de comunicação; o rptTimer configura o tempo de retransmissão da mensagem e o txTimer configura o tempo de transmissão. Essa função deve ser chamada antes das funções lon_nv_fetch e lon_query_status;

- void lon_set_dest_addr_id(int domainIndex, int subnet, String neuronId, int retry, int rptTimer, int txTimer): similar à função lon_set_dest_addr_sn, com a diferença que configura o endereço de destino da mensagem através do Neuronld do dispositivo; mesmo assim deverá ser informado o domínio e a subnet do dispositivo;

- void lon_set_dest_addr_bc(int domainIndex, int subnet, int backlog, int retry, int rptTimer, int txTimer): configura um endereço de broadcast para o envio das mensagens;

- void lon_set_dest_addr_lc() : configura o endereço de destino como sendo o próprio nó, sendo uma espécie de configuração "localhost";

- int lon_nv_fetch(int nvIndex): obtém o valor de uma variável de rede de um dispositivo endereçado pelos métodos acima e com índice nvIndex; 
- int [] lon_query_status(): traz em um array todas as informações de diagnóstico do dispositivo. $O$ item 3.5 deste documento apresenta mais detalhes sobre a mensagem.

O Apêndice A apresenta um roteiro de como incorporar rotinas de uma $D L L$ em uma classe Java utilizando os métodos nativos Java Native Interface (JNI).

Esta classe será muito útil para futuros trabalhos utilizando a tecnologia Java, como por exemplo, para o trabalho de conexão remota à rede LonWorks através de applets na Internet.

\subsection{IMPLEMENTAÇÃO}

No desenvolvimento do agente proxy SNMP, foram utilizados uma Network Interface Card - Internet Protocol (NIC-IP) da empresa LoyTec (LOYTEC, 2005), que é responsável por realizar a interface entre o computador e a rede LonWorks, e dois narizes eletrônicos para redes LonWorks, da empresa PureChoice (PURECHOICE, 2007). Como ferramentas de desenvolvimento foram utilizadas as Integrated Development Environment (IDE) Java Studio Enterprise $8^{1}$ e Dev-C++ ${ }^{2}$. Para a realização dos testes de comunicação com o agente SNMP e desenvolvimento da LON-NODE-MIB, foi utilizado o AdventNet Agent Toolkit Java Edition 6, da empresa AdventNet (ADVENTNET, 2007), que reúne um editor de MIBs SNMP e um MIB browser, capaz de requisitar informações de um agente SNMP e receber Traps.

Inicialmente verificou-se o funcionamento da API AgenteAPI, criando-se um agente SNMP capaz de implementar o objeto MIB SysContact (.1.3.6.1.2.1.1.4.0); desta forma, ao realizar um comando GetRequest para o objeto MIB SysContact, o agente deveria retornar o contato do desenvolvedor do agente, como apresentado na Figura 19. Esse teste permitiu um melhor conhecimento da API e possibilitou a validação da utilização da mesma para o desenvolvimento do agente proxy SNMP.

\footnotetext{
${ }_{2}^{1}$ Ferramenta gratuita de desenvolvimento em linguagem Java da empresa Sun Mycrosystems.

${ }^{2}$ Ferramenta gratuita de desenvolvimento em linguagens $\mathrm{C}$ e $\mathrm{C}++$ da empresa BloodShedSoftware
} 
Figura 19. Teste de requisição do objeto MIB SysContact implementado no agente.

Verificado que o mecanismo de comunicação SNMP já estava funcionando corretamente, foi necessário realizar os testes de comunicação com a rede LonWorks. Para isso, implementou-se a classe gateway, que foi responsável por encapsular a comunicação com a rede, através dos métodos disponíveis na $D L L$ LonAccess, já anteriormente implementados e testados em Canovas et al. (2006). Nesta etapa, o objetivo principal era resgatar as informações do dispositivo LonWorks e validar a implementação da classe gateway em linguagem Java; porém os métodos existentes na $D L L$ não cobriam a parte de diagnóstico da rede, sendo necessário realizar uma melhoria na $D L L$, de maneira que ela pudesse ser utilizada pelo agente proxy SNMP. A Figura 20 apresenta as informações de diagnóstico resgatadas pela classe gateway.

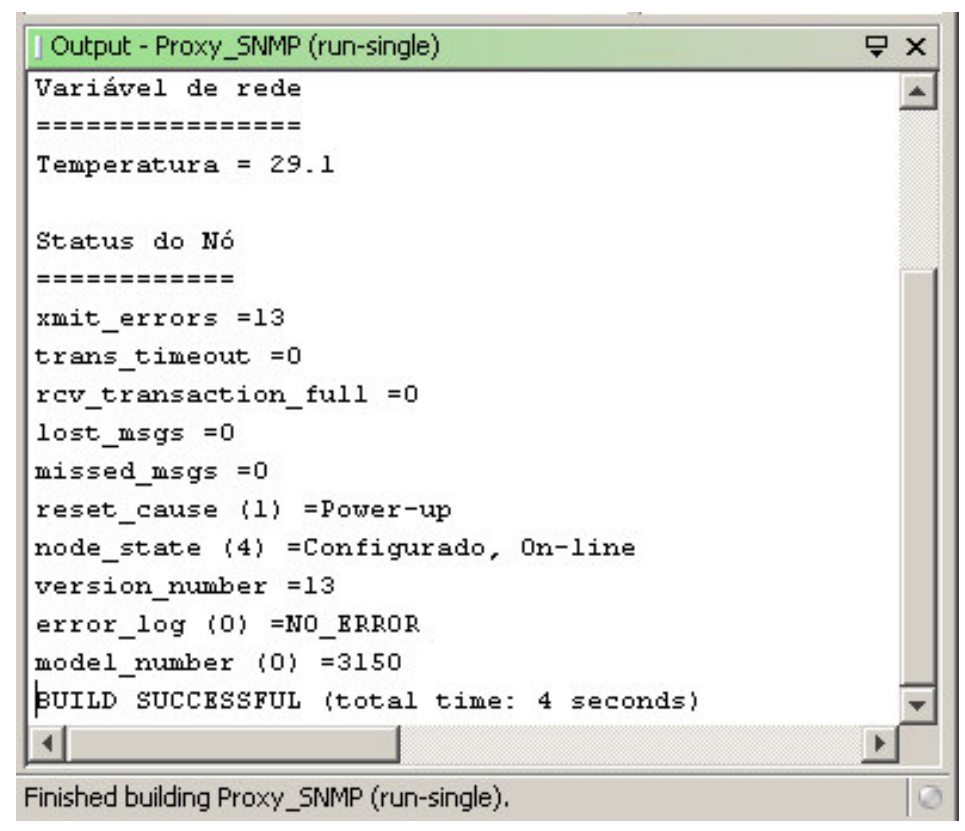

Figura 20. Teste de requisição do status do dispositivo através da classe gateway. 
A partir do momento que foi validada a comunicação com a rede LonWorks, tornou-se mais fácil testar a implementação deste novo método. As informações resgatadas por essa nova funcionalidade foram comparadas com as informações acessadas através da utilização do software NodeUtil da empresa Echelon (ECHELON, 2007a), a Figura 21 apresenta o status do mesmo dispositivo através desse software. Assim, foi possível validar o novo método desenvolvido para capturar as informações de diagnóstico do dispositivo que estava funcionando e pronto para ser utilizado na composição do agente proxy SNMP.

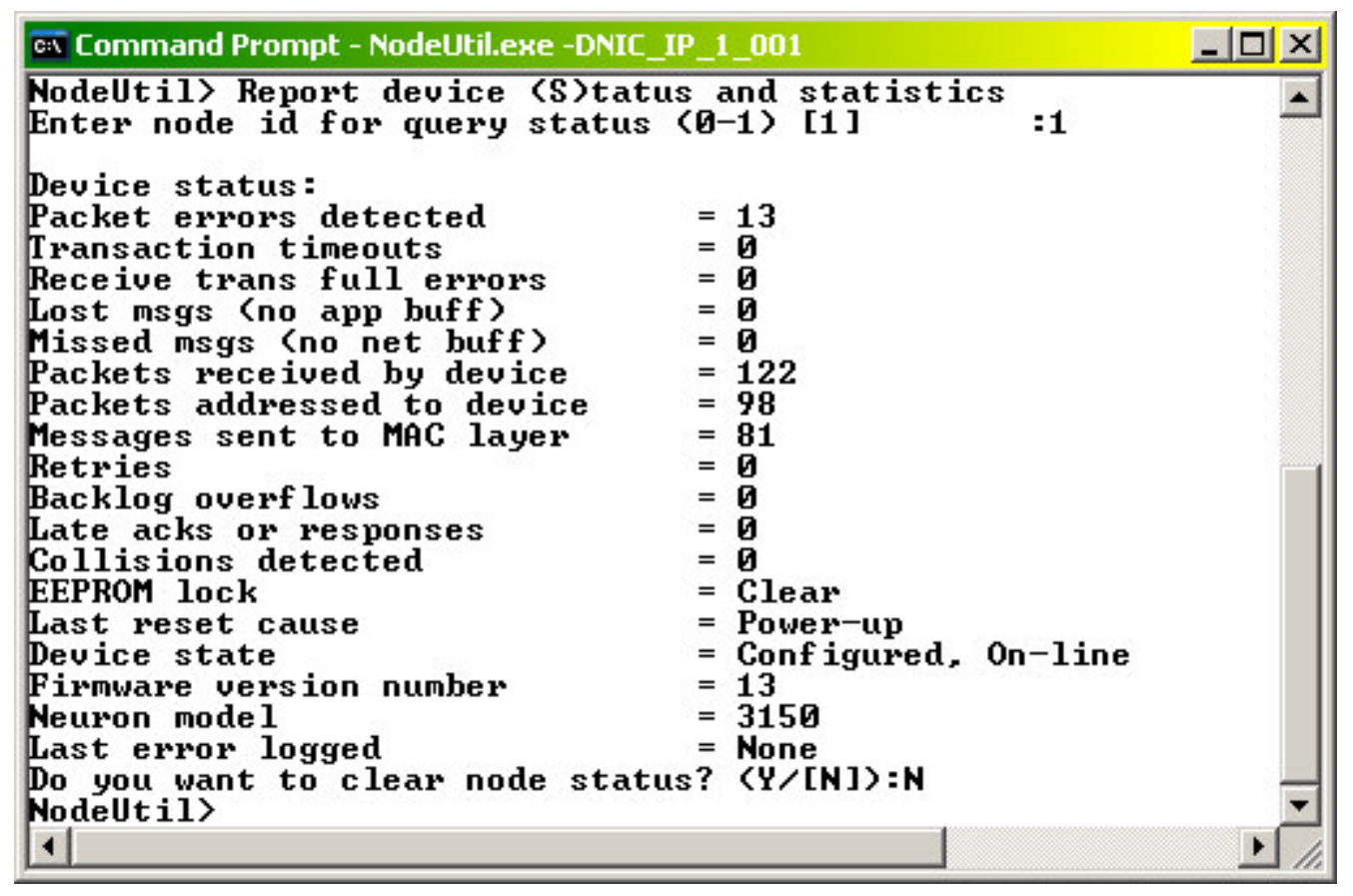

Figura 21. Requisição do status do dispositivo através do programa NodeUtil.

Comprovada a viabilidade de comunicação com gerente SNMP e com a rede LonWorks, restava somente realizar a integração dos dois mecanismos, que foi feito através de um grupo de classes responsáveis por implementar cada um dos objetos da LON-NODE-MIB, encapsuladas no package MibNode, como mostra a Figura 18, e pelo desenvolvimento da classe Node.

Essa classe foi desenvolvida para armazenar as informações relacionadas a cada dispositivo a ser monitorado e ser responsável por realizar requisições periódicas a rede LonWorks, com objetivo de manter essas informações atualizadas.

O agente, ao receber uma requisição de um objeto MIB do gerente SNMP, verifica qual instância que está relacionada para atender tal requisição. A classe 
dessa instância já foi previamente programada para buscar a informação correspondente na classe Node, sendo capaz de retornar a informação para o gerente SNMP no formato correto, sem a necessidade de realizar uma nova requisição à rede LonWorks.

A Figura 22 apresenta o teste de comunicação realizado através do MIB browser com o objetivo de capturar as informações de diagnóstico dos dois dispositivos monitorados pelo agente.

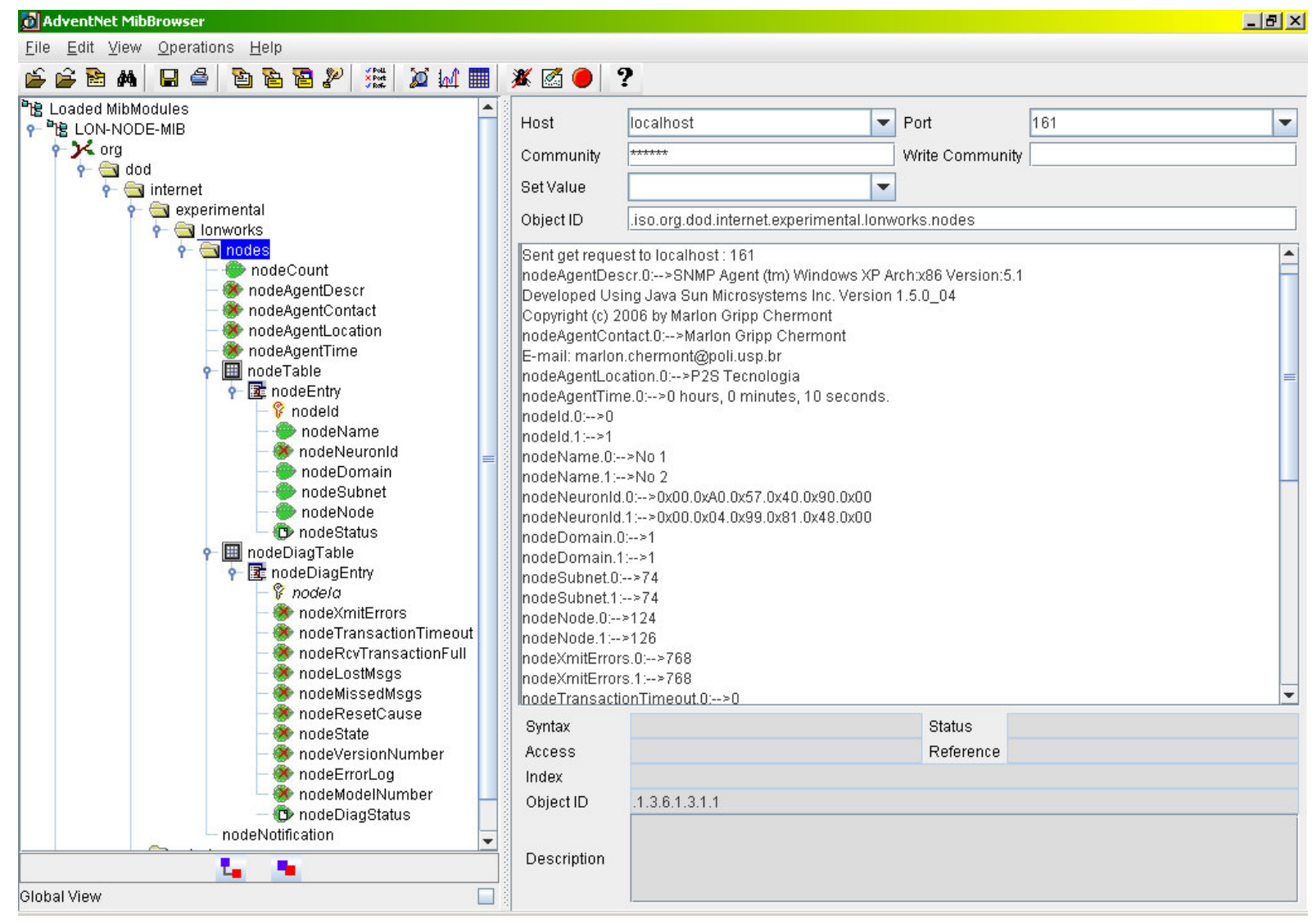

Figura 22. Tela do programa MibBrowser consultando as informações do agente proxy SNMP.

\subsection{TESTES E ANÁLISES}

Nesta seção é apresentada uma série de experimentos realizados para diferentes cenários com o objetivo de analisar a eficiência do agente desenvolvido. Para isso o agente foi executado monitorando certo número de dispositivos e com um determinado tempo de varredura. Durante um período de cinco minutos foi computado o tempo gasto para obter os dados atualizados, o tempo gasto para 
requisitar a informação de status de um dispositivo LonWorks e obter uma resposta válida. Também foi calculado o tempo de espera gerado para uma resposta de falha, permitindo também verificar a porcentagem de mensagens com falhas geradas durante o experimento.

No primeiro cenário, foram utilizados dois dispositivos Nose $^{3}$ e uma taxa de varredura de $2.000 \mathrm{~ms}$, escolhida arbitrariamente. Como pode ser observado no gráfico da Figura 23, a maioria dos nós demorou cerca de 2.100 ms para ser atualizada, pois o tempo médio de resposta da requisição era de $110 \mathrm{~ms}$. O gráfico apresenta também o número de vezes que o dispositivo demorou uma determinada quantidade de tempo para realizar uma função; portanto, pode-se observar onde está a maior concentração de tempo na realização de uma determinada função, possibilitando inferir o tempo médio utilizado para mesma no experimento.

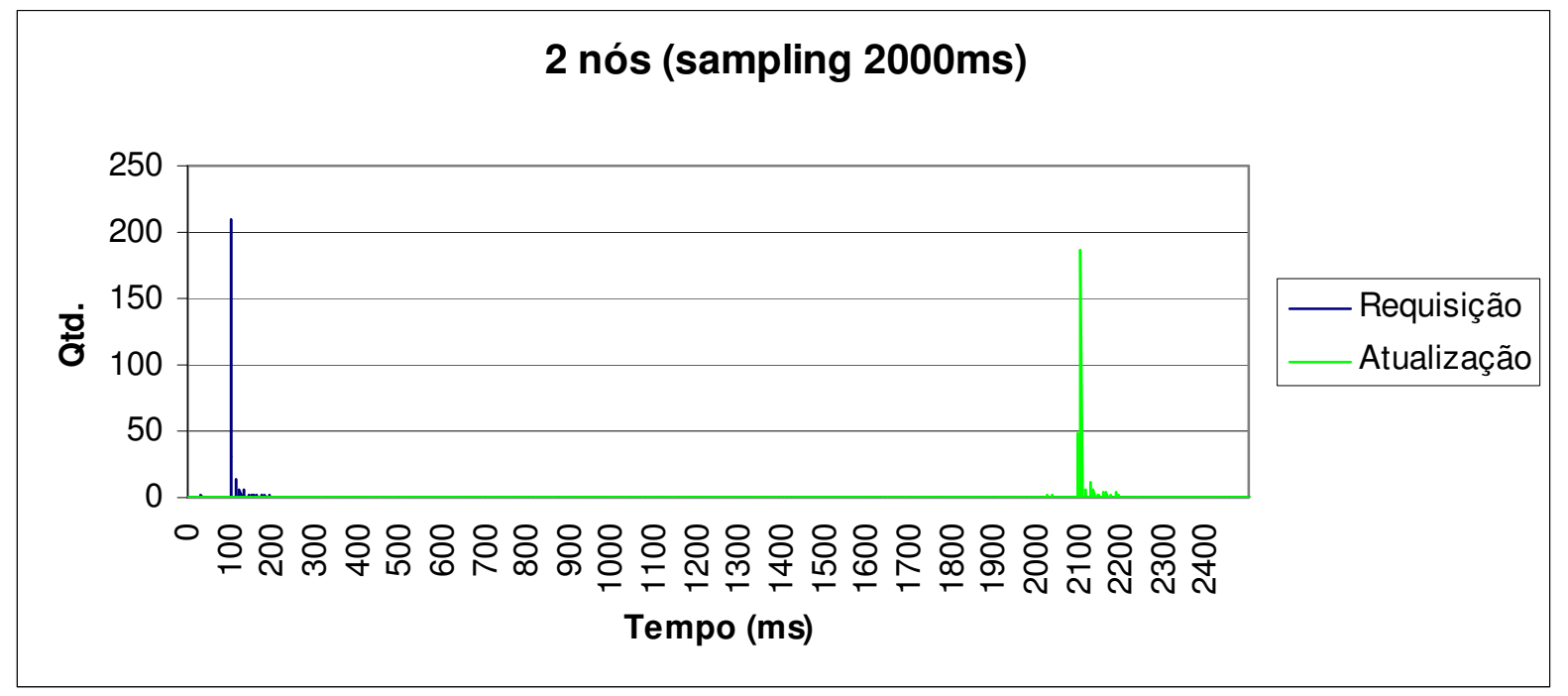

Figura 23. Tempo médio de requisição e atualização em uma rede com dois nós e taxa de varredura de $2.000 \mathrm{~ms}$.

No primeiro cenário, 99,65\% das requisições retornaram respostas válidas e somente uma resposta estourou o tempo de espera de $5.868 \mathrm{~ms}$ retornando valor "vazio", o que aparenta ser um resultado positivo; porém nem sempre o agente poderá operar monitorando apenas dois dispositivos.

Considerando o número máximo de dispositivos num mesmo canal, criou-se mais um cenário onde foi simulada a utilização de 64 dispositivos com a mesma taxa

\footnotetext{
${ }^{3}$ Dispositivo LonWorks da empresa PureChoice que possui 5 sensores (Temperatura, Umidade, Monóxido de Carbono (CO), Dióxido de Carbono $\left(\mathrm{CO}_{2}\right)$ e Componentes Orgânicos e Voláteis (VOC))
} 
de varredura $(2.000 \mathrm{~ms})$. Como não existiam 64 dispositivos diferentes disponíveis para os testes, os endereços dos dois nós disponíveis foram replicados até atingir 64 dispositivos monitorados pelo agente.

Neste cenário aproximadamente $82 \%$ das requisições realizadas retornaram valores "vazios", reduzindo a eficiência do agente e aumentando o tempo necessário para ter as informações do dispositivo atualizadas, com é apresentado no gráfico da Figura 24.

Alguns dispositivos demoraram mais do que $250 \mathrm{~s}$ para ter sua informação atualizada, apesar da taxa de varredura ser de $2.000 \mathrm{~ms}$, apenas $33 \%$ das atualizações ocorreram em menos do que 2,5 s.

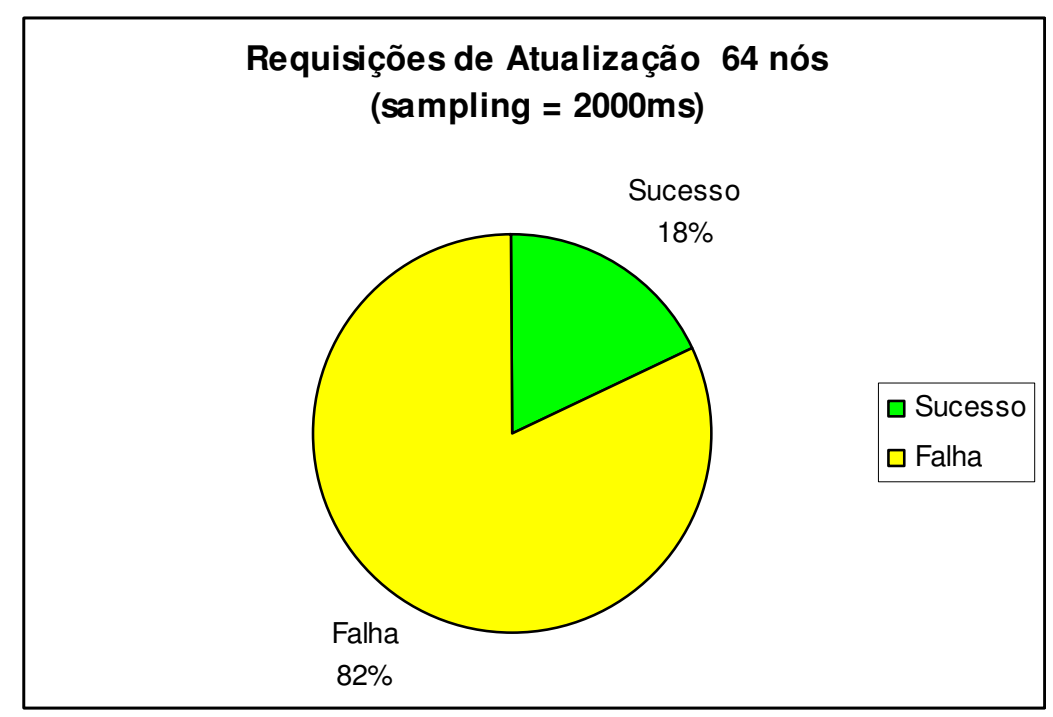

Figura 24. Porcentagem de falha na mensagem de requisição de atualização do dispositivo LonWorks.

O baixo desempenho do segundo cenário reflete o excesso de requisições geradas num curto período de tempo. Como cada um dos dispositivos comporta-se de maneira independente dentro do agente, as requisições são geradas em instantes de tempos muito próximos. Neste caso, pode-se estimar cerca de 32 requisições por segundo, exigindo um tempo de resposta de 31,25 ms. Como observado no primeiro cenário, o tempo médio de uma resposta válida foi de 110 ms: dessa forma o tempo de varredura ideal seria de $7.040 \mathrm{~ms}$, supondo que requisições fossem realizadas de maneira seqüenciais, porém, isso não acontece nessa implementação do agente. 
Para avaliar o impacto desta arquitetura, onde cada nó automaticamente, ao ser instanciado, realiza uma requisição na rede LonWorks, foi realizado um experimento, considerando um tempo de varredura de 7.040 ms. Houve uma melhoria em relação ao número de requisições válidas; porém ainda apresenta um baixo desempenho com cerca de $74 \%$ de falhas.

Como se pode observar no gráfico da Figura 25, existe uma concentração em torno do instante $7.500 \mathrm{~ms}$; porém grande parte das amostras está distribuída durante o período do experimento (300.000 ms), com apenas $44 \%$ das atualizações sendo realizadas em menos de $7,5 \mathrm{~s}$.

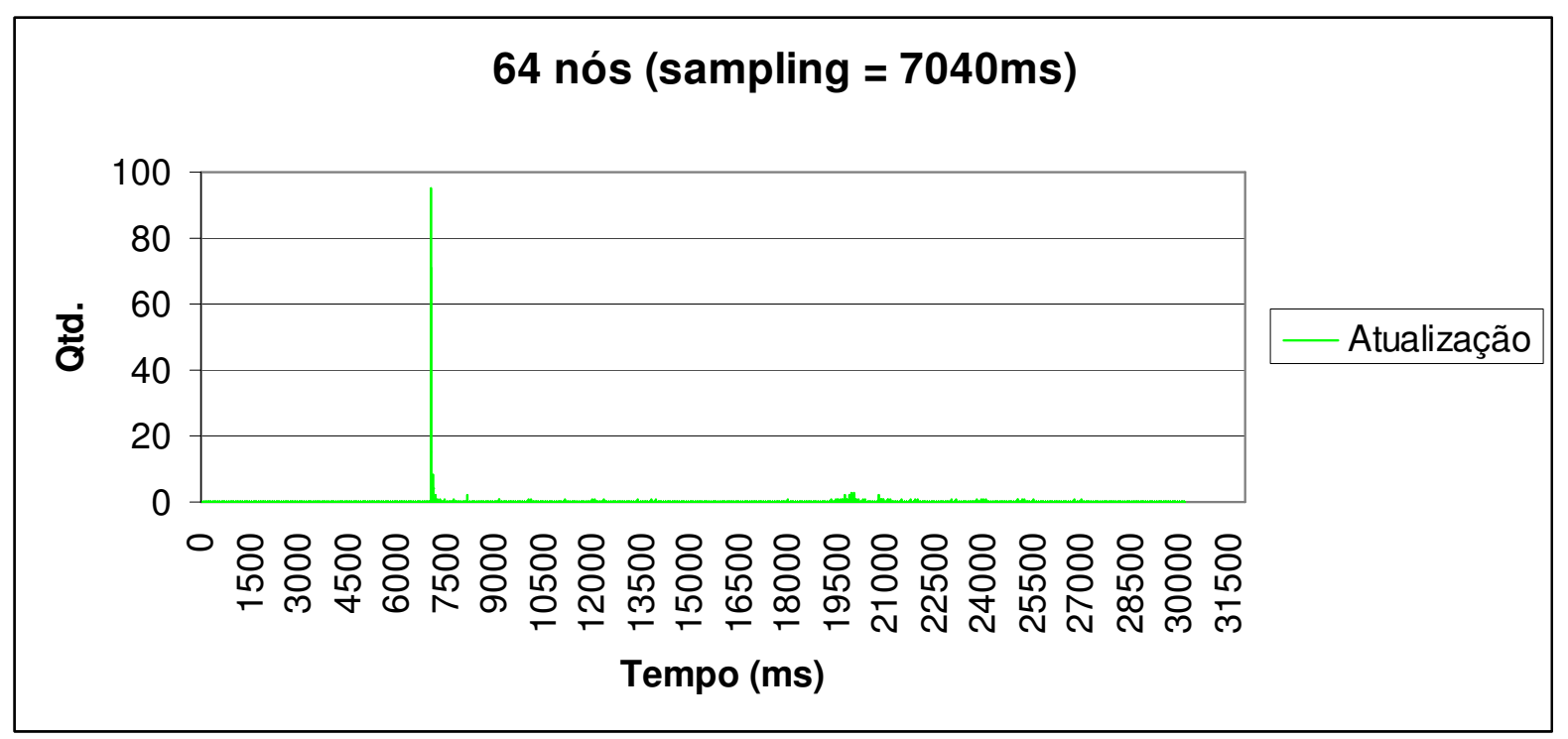

Figura 25. Tempo médio de atualização em uma rede com 64 nós e taxa de varredura de $7.040 \mathrm{~ms}$.

Foram realizadas duas melhorias no agente, com o objetivo de aumentar 0 seu desempenho. A primeira melhoria foi impedir que um nó tentasse requisitar informações da rede ao ser instanciado: dessa forma, impediu-se que os nós requisitassem informações em instantes muito próximos, pois à medida que cada nó era instanciado dentro do agente, ele aguardava um instante tempo $\varphi$ representado por $\mathrm{N}^{\star} 150 \mathrm{~ms}$, onde $\mathrm{N}$ é o identificador do dispositivo instanciado.

A outra alteração realizada prevê a utilização do mesmo tempo $\varphi$ de espera, no caso de falha de requisição de um nó. Isso permitiu reduzir a probabilidade de um nó requisitar informações da rede em instantes muito próximos de um outro. Conforme apresentado no gráfico da Figura 26, ocorreu uma melhoria significativa no aproveitamento do agente, onde $59 \%$ das requisições foram válidas e o maior 
intervalo de atualização foi de $132 \mathrm{~s}$, apesar de existir um tempo muito alto aproximadamente $61 \%$ das atualizações ocorreram em menos de 7,5 segundos - o que representa um ganho de eficiência de $72 \%$ em relação ao tempo médio de atualização dos dispositivos da versão anterior.

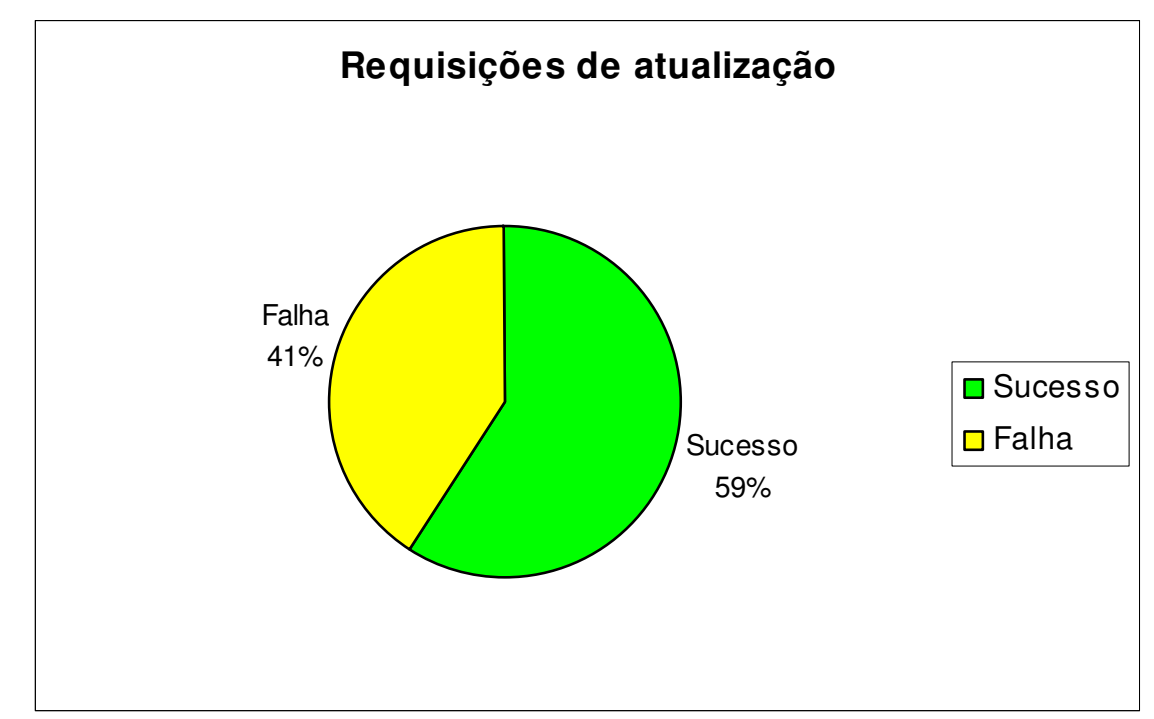

Figura 26. Porcentagem de sucesso na mensagem de requisição de atualização do dispositivo LonWorks com melhorias no agente. 


\section{DISCUSSÃO DOS RESULTADOS E CONCLUSÃO}

\subsection{CONCLUSÕES}

Nesse trabalho foi desenvolvido o protótipo de uma solução aberta para o gerenciamento de uma rede de automação integrada a uma rede corporativa, que pode ser considerada como um embrião para o desenvolvimento de uma ferramenta capaz de auxiliar o gerenciamento de uma rede LonWorks.

Através do desenvolvimento do agente proxy SNMP, foi possível integrar diferentes tecnologias em uma única solução, propiciando um ganho de funcionalidade e de recursos, em uma área que é dominada por soluções comerciais, como é a área de automação predial.

Pode-se destacar a área da automação predial como uma das mais favorecidas por este trabalho, pois atualmente, dentro dos prédios modernos, já existe uma infra-estrutura de rede de dados, ao lado de uma outra infra-estrutura focada no conforto, bem-estar das pessoas e na economia de recursos. Cada uma dessas redes utiliza a tecnologia mais adequada para fornecer a solução; porém é cada vez mais forte a necessidade de uma convergência dessas tecnologias, a fim de reduzir ainda mais os custos atrelados à manutenção dessas diferentes infraestruturas.

Assim, pode-se destacar como um dos principais resultados desse trabalho, a possível solução para essa convergência, de maneira a viabilizar o agrupamento das equipes de manutenção das redes de dados, com a equipe de manutenção da infra-estrutura do prédio.

De uma maneira mais ampla, pode-se considerar essa ferramenta como uma possível alternativa para a pesquisa do gerenciamento de redes de automação baseada na tecnologia LonWorks; já que todas as ferramentas encontradas no mercado são de uso comercial. Isso poderá auxiliar na avaliação de desempenho da rede, o levantamento de novas necessidades e a análise de redes criada para testes.

Os testes de desempenho do agente indicam a necessidade de melhorias; porém o fato de se utilizar apenas dois dispositivos para a simulação da rede pode ter impactado nos tempos de respostas adquiridos, dado que os mesmos 
dispositivos deveriam responder a requisições diferentes em tempos muito curtos, o que pode ter provocado um atraso no envio das respostas. $\mathrm{E} O$ fato de a rede utilizada para os testes ter um pequeno comprimento e não possuir repetidores também pode ter impactado positivamente nos tempos de respostas adquiridos, pois os tráfegos de pacotes sofreram menor interferência.

Assim sendo, não foi possível diagnosticar o baixo desempenho encontrado e definir se ele irá se repetir ao ser utilizada uma rede com diferentes dispositivos. Porém, espera-se uma queda no desempenho, pois o comprimento da rede e a utilização de repetidores devem provocar um aumento nos tempos de respostas maior que o ganho de tempo adquirido pela utilização de diferentes dispositivos.

Fica em aberto, também, a definição de uma solução capaz de reunir o gerenciamento dos mais de 32 mil dispositivos que podem ser utilizados em um mesmo domínio. Certamente este trabalho pode auxiliar na criação dessa solução, ao permitir que a rede seja dividida em pequenas partes, que podem ser monitoradas em intervalos maiores de tempo.

A utilização da linguagem Java para o desenvolvimento do agente SNMP ainda propiciou que, através da substituição da DLL LonAccess de acesso a rede LonWorks, se torne possível disponibilizar o agente para ser executado em outros sistemas operacionais.

\subsection{CONTRIBUIÇÕES}

Pode-se destacar como sendo contribuições relevantes deste trabalho para as pesquisas realizadas no Laboratório de Automação Agrícola da USP, os seguintes aspectos:

- Desenvolvimento do gateway Java com a rede LonWorks: essa interface de comunicação irá auxiliar a criação de projetos relacionados aos laboratórios virtuais desenvolvidos no projeto TIDIA/Kyatera (TIDIA, 2007), apoiado pela FAPESP;

- Implementação da mensagem query_status na DLL LonAccess: 0 desenvolvimento desta nova mensagem contribui ainda mais com o aprimoramento da estrutura de comunicação com a rede LonWorks disponível para ser utilizada pelas pesquisas do Laboratório de Automação Agrícola da Escola Politécnica da USP; 
- MIB LonWorks: criação de um protótipo de MIB Lonworks que pode ser utilizada na integração da tecnologia com o gerenciamento SNMP apresentada no Apêndice B.

\subsection{TRABALHOS FUTUROS E MELHORIAS}

Após as avaliações experimentais, identificaram-se algumas melhorias que poderão ser implementadas no agente, que são:

- Implementação de outras mensagens de gerenciamento disponíveis no protocolo LonTalk, como por exemplo, a mensagem read_memory que é capaz de retornar algumas mensagens a mais de diagnóstico da rede;

- Melhoria do controle de atualização dos dispositivos da rede. Atualmente os dispositivos tentam se comunicar com a rede de maneira aleatória, isso acaba limitando o número de dispositivos que podem ser monitorados pelo agente, pois não existe um controle e nem uma organização das requisições realizadas. Seria interessante implementar um controle único de atualizações, onde as requisições pudessem ser realizadas de maneira seqüencial e que reduzisse o número de mensagens com falha;

- Criação de uma interface de inclusão de novos dispositivos e uma visualização da arquitetura da rede LonWorks onde fosse possível visualizar o tráfego de cada segmento da rede;

- Permissão da inclusão de um novo dispositivo no sistema através da utilização do pino de serviço do dispositivo, que gera uma mensagem de broadcast padrão;

- Desenvolvimento de uma rotina de acesso à rede LonWorks em Linux, que propicie a execução do agente nesse sistema operacional;

- Ampliação da estrutura disponível do agente para possibilitar o gerenciamento dos mais de 32 mil dispositivos que podem fazer parte de um mesmo domínio;

- Utilização da estrutura disponível para monitorar e supervisionar a planta de automação além de realizar o gerenciamento. Isso permitiria 
a utilização das ferramentas disponíveis para o gerenciamento SNMP para realização da supervisão da planta ${ }^{4}$.

\subsection{CONSIDERAÇÕES FINAIS}

As construções de prédios inteligentes, capazes de controlar o consumo de energia elétrica, água e utilização dos sistemas de ar-condicionado, estão se tornando cada vez mais uma necessidade do que simplesmente um diferencial de mercado, devido ao fato de os recursos estarem se tornando mais escassos e menos baratos.

Por outro lado, a informática e as redes de computadores estão cada vez mais freqüentes e é cada vez mais fácil encontrar em novos prédios uma infraestrutura compartilhada, o que acaba exigindo a dedicação de uma equipe responsável pelo gerenciamento da rede.

Aproveitar as equipes de manutenção do prédio para cuidar de toda a infraestrutura acaba sendo um desejo ao se observar a complexidade das tecnologias implantadas, dessa forma as pesquisas nas convergências tecnológicas toma um destaque relevante, sem falar das possibilidades de compartilhamento de informações.

Esse trabalho espera ter colaborado com uma proposta de solução para integrar tecnologias, que cada vez mais convivem lado a lado. As pesquisas nessa área podem auxiliar no questionamento do desenvolvimento de novas soluções integradas à área da automação predial.

\footnotetext{
${ }^{4}$ Esta proposta está sendo implementada por Marcelo Succi de Jesus Ferreira em seu trabalho de mestrado, e por Leonardo Bessa em seu trabalho de iniciação científica, orientados pelo Prof. Dr. Pedro Luiz Pizzigatti Corrêa do Departamento de Engenharia de Computação e Sistemas Digitais da Escola Politécnica da Universidade de São Paulo.
} 


\section{REFERÊNCIAS}

ADVENTNET. Site da empresa AdventNet. Disponível em: <http://www.adventnet.com>. Acesso em: 10 fev. 2007.

CANOVAS, S.R.M.; CHERMONT, M.G. RemoteLon: Sistema Supervisório Remoto para Redes LonWorks. 2003. 101p. Monografia (Projeto de Formatura) Escola Politécnica, Universidade de São Paulo. São Paulo, 2003.

CANOVAS, S.R.M.; CHERMONT, M.G.; CUGNASCA, C.E.; Remote Monitoring and Actuation Based on LonWorks ${ }^{\circledR}$ Technology. In: EFITA/WCCA JOINT CONGRESS ON IT IN AGRICULTURE, 2005, Vila Real, Portugal. Proceedings..., Vila Real, Portugal: Universidade de Trás-os-Montes e Alto Douro, 2005. v. 1, p. 812-819.

CANOVAS, S.R.M.; CHERMONT, M.G.; CUGNASCA, C.E.; PEREIRA, G.A.; Remote Monitoring Based on LonWorks Technology: A Greenhouse Application. In: WORLD CONGRESS ON COMPUTERS IN AGRICULTURE, 4., 2006, Orlando, USA. Proceedings... Vila Real, Portugal: Universidade de Trás-os-Montes e Alto Douro, 2006. v. 1, p. 122-127.

CANOVAS, S.R.M. Integração entre redes LonWorks e redes IP: aplicações, requisitos e soluções. 2006. 196 p. Dissertação (Mestrado) - Escola Politécnica, Universidade de São Paulo, São Paulo, 2006.

CHERMONT, M.G.; ALVES FILHO, M.S.; CANOVAS, S.R.M.; CUGNASCA, C.E.; Automação Predial Aplica em Estufas. In: Congresso Brasileiro de Agroinformática, SBI-AGRO, 5., 2005, Londrina. Anais.... Londrina: Universidade Estadual de Londrina, 2005. 1 CD-ROM.

INTERNETWORKING TECHNOLOGIES HANDBOOK. Chapter 56 - Simple Network Management Protocol. Disponível em: <http://www.cisco.com/ univercd/cc/td/doc/cisintwk/ito_doc/snmp.pdf>. Acesso em: 22 jan. 2006.

SIMPLE NETWORK MANAGEMENT PROTOCOL (SNMP) SNMP Background Disponível em: <http://www.cisco.com/warp/public/535/3.html>. Acesso em: 14 ago. 2006.

ECHELON. Site da empresa Echelon Corporation. (a) Disponível em: <http://www.echelon.com>. Acesso em: 20 fev. 2007.

Echelon technology adopted as european building control standard. (b) Disponível em: <http://www.echelon.com/company/press/2005/ahr2005.htm>. Acesso em: 09 jan. 2007. /Matéria sobre a adoção de LonWorks como padrão europeu./

Introduction to the LONWORKS $\AA$ System. Palo Alto, Echelon, 1999 (Relatório 078-0183-01A). 
. LonMark Layer 1-6 Interoperability Guidelines. Palo Alto, Echelon, 2002 (a) (Relatório 078-0120-01F).

. LonMark Application-Layer Interoperability Guidelines. Palo Alto, Echelon, 2002 (b) (Relatório 078-0120-01).

IHS/GLOBAL. Site da empresa IHS fornecedora de informações técnicas. Disponível em: <http://global.ihs.com/>. Acesso em: 16 jan. 2007.

INS. Site da empresa Industrial Networking Solutions. Disponível em: <http://www.industrialnetworking.com/products/network_management/>. Acesso em: 14 ago. 2006.

JNI - JAVA NATIVE INTERFACE. Site com um tutorial de programação JNI. Disponível em: <http://java.sun.com/docs/books/jni/html/jniTOC.html>. Acesso em: 14 jan. 2007.

KASTNER, W.; NEUGSCHWANDTNER, G.; SOUCEK, S.; NEWMANN, H.M. Communication Systems for Building Automation and Control. Proceedings of the IEEE, v. 93, n. 6, p. 1178-1203, jun. 2005.

KNIZAK, M; KUNES, M.; MANNINGER, M.; SAUTER, T.; Applying Internet Management Standards to Fieldbus Systems. In: IEEE INTERNATIONAL WORKSHOP ON FACTORY COMMUNICATION SYSTEMS, 1997, Barcelona, Espanha. Proceedings..., 1997. p. 309-315.

KUNES, M.; SAUTER, T.; Fieldbus-Internet Connectivity: The SNMP Approach. IEEE Transactions on Industrial Electronics, v. 48, n. 6, p. 1248-1256, dez. 2001.

LAA - LABORATÓRIO DE AUTOMAÇÃO AGRÍCOLA DA ESCOLA POLITÉCNICA DA USP. Site do LAA. Disponível em: <http://laa.pcs.usp.br>. Acesso em: 27 nov. 2006.

LOPES, R. F. Desenvolvimento de um agente SNMP multi-plataforma. 2003. 125p. Monografia (Trabalho de Conclusão de Curso) - Universidade Federal do Maranhão. São Luís - MA, 2003.

LOPES, R. P. S. C. Gestão distribuída em SNMP. 2002. 191p. Dissertação (Doutorado) - Departamento de Electrónica e de Telecomunicações, Universidade de Aveiro. Portugal, 2002.

LOPES, R. P.; OLIVEIRA, J. AgentAPI: an API for the Development of Managed Agents. In: International Conference On Enterprise Information Systems, 5., 2003, Angers, França. Proceedings..., Angers, França. 23-26 abr. 2003.

LOYTEC. Site da empresa Loytec. Disponível em: <http://www.loytec.com>. Acesso em: 29 maio 2006.

MAHALIK, N.G.P.C.; LEE, S.K. A study on production line automation with LonWorks $^{\mathrm{TM}}$ control networks. Computer Standards \& Interfaces, n.24, p.21-27, 2002. 
MAURO, D.; SCHMIDT, K. Essential SNMP. 2.ed. O'Reilly, 2005. 460p.

PEREIRA, G.A. Sistema experimental de monitoramento e controle para casas de vegetação baseado em redes de controle distribuído LonWorks. 2006. 125 f. Dissertação (Mestrado) - Escola Politécnica, Universidade de São Paulo, São Paulo, 2006.

PURECHOICE. Site da empresa PureChoice. Disponível em: <http://www.purechoice.com>. Acesso em: 10 fev. 2007.

RAD University. Site com um tutorial de SNMP. Disponível em: <http://www2.rad.com/networks/1999/snmp/index.htm>. Acesso em: 22 jan. 2006.

STALLINGS, W. SNMP, SNMPv2, AND RMON: The Practical Network Management. 2.ed. Reading, Addson-Wesley, 1996. 478p.

TIDIA - Tecnologia da Informação no Desenvolvimento da Internet Avançada. Disponível em: <http://www.tidia.fapesp.br/>. Acesso em: 19 jan. 2007.

TOSHIBA. Neuron Chip Data Book. Toshiba, EUA, 1995. 


\section{REFERÊNCIAS RECOMENDADAS}

ALVES FILHO, M.S.; CUGNASCA, C.E.; DIAS, E.M. LonWorks: Inovação Tecnológica para Automação Residencial, Predial e Industrial. In: CONAI CONGRESSO E EXPOSIÇÃO INTERNACIONAL DE AUTOMAÇÃO, 11., São Paulo. 2004. Programação Temária do Congresso. São Paulo: Conai, 2004. Disponível em: <http://www.conai.com.br/palestras2004/13_05_04_15h30_Miguel.zip>. Acesso em: 13 set.2005.

BOLZANI, C.A.M. Residências Inteligentes. 1.ed. São Paulo: Editora Livraria da Física, 2004. 332p.

CANOVAS, S.R.M; CHERMONT, M.G.; ALVES FILHO, M.S.; CUGNASCA, C.E.; Sistema Supervisório para Redes LonWorks. In: CONAI - CONGRESSO E EXPOSIÇÃO INTERNACIONAL DE AUTOMAÇÃO, 11., São Paulo. 2004. Programação Temária do Congresso. São Paulo: Conai, 2004. Disponível em: <http://www.conai.com.br/palestras2004/13_05_04_15h_Marlon.zip>. Acesso em: 13 set. 2005.

CLEMENTI, S. Metodologia para especificação e implementação de solução de gerenciamento em redes de computadores corporativas. 1999.163 p. Dissertação (Mestrado) - Escola Politécnica, Universidade de São Paulo. São Paulo, 1999.

CONCEITO TECNOLOGIA. Site da empresa Conceito Tecnologia. Disponível em: <http://www.conceitotecnologia.com.br>. Acesso em: 13 set. 2005.

ECHELON. LonWorks Host Application Programmer's Guide. Palo Alto, Echelon, 1993 (Relatório 078-0016-01B).

LEE, J.; HSU, P. Design and Implementation of the SNMP Agents for Remote Monitoring and Control via UML and Petri Nets. IEEE Transactions on Control Systems Technology, v. 12, n. 2, p. 293-302, mar. 2004.

FÓRUM LONWORS BRASIL. Fórum de discussão da tecnologia LonWorks. Disponível em: <http://www.lonbrasil.com.br>. Acesso em: 12 jan. 2007.

NETWORK WORKING GROUP. A Simple Network Management Protocol (SNMP) - RFC1157. 1990. Disponível em <http://www.ietf.org/rfc/rfc1157.txt>. Acessado em 12 jan. 2007.

P2S TECNOLOGIA. Site da empresa P2S Tecnologia. Disponível em: <http://www.p2s.com.br>. Acesso em: 28 jul. 2006.

PENG, H.; YANG, J. Decision support to the application of intelligent building technologies. Renewable Energy, v. 22, p. 67-77, 2001.

REITER, H.; KRAL, C. Interaction between Java ${ }^{\mathrm{TM}}$ and LonWorks ${ }^{\circledR}$, Factory Communication Systems In: IEEE INTERNATIONAL WORKSHOP ON FACTORY 
COMMUNICATION SYSTEMS, 1997, Barcelona, Espanha. Proceedings..., Barcelona, Espanha, 1997. p. 335-340.

TANENBAUM, A.S. Computer Networks. 3.ed. Upper Saddle River: Prentice Hall, 1996. 813p.

TOPALIS, E.; ORPHANOS, G.; KOUBIAS, S.; PAPADOPOULOS, G. A Generic Network Management Architecture Targeted to Support Home Automation Networks And Home Internet Connectivity. IEEE Transactions on Consumer Eletronics, v. 46, n. 1, p. 44-51, fev. 2000. 


\section{APÊNDICE A - EXEMPLO DE IMPLEMENTAÇÃO JNI}

Este apêndice tem como objetivo fornecer um pequeno roteiro de implementação da estrutura Java Native Interface (JNI) do Java. Ele foi baseado no tutorial disponível no JNI (2007).

A Figura 27 ilustra o processo de programação de uma simples aplicação escrita em linguagem Java que chama uma função implementada em linguagem $C$, que imprime na tela a mensagem "Hello World!".

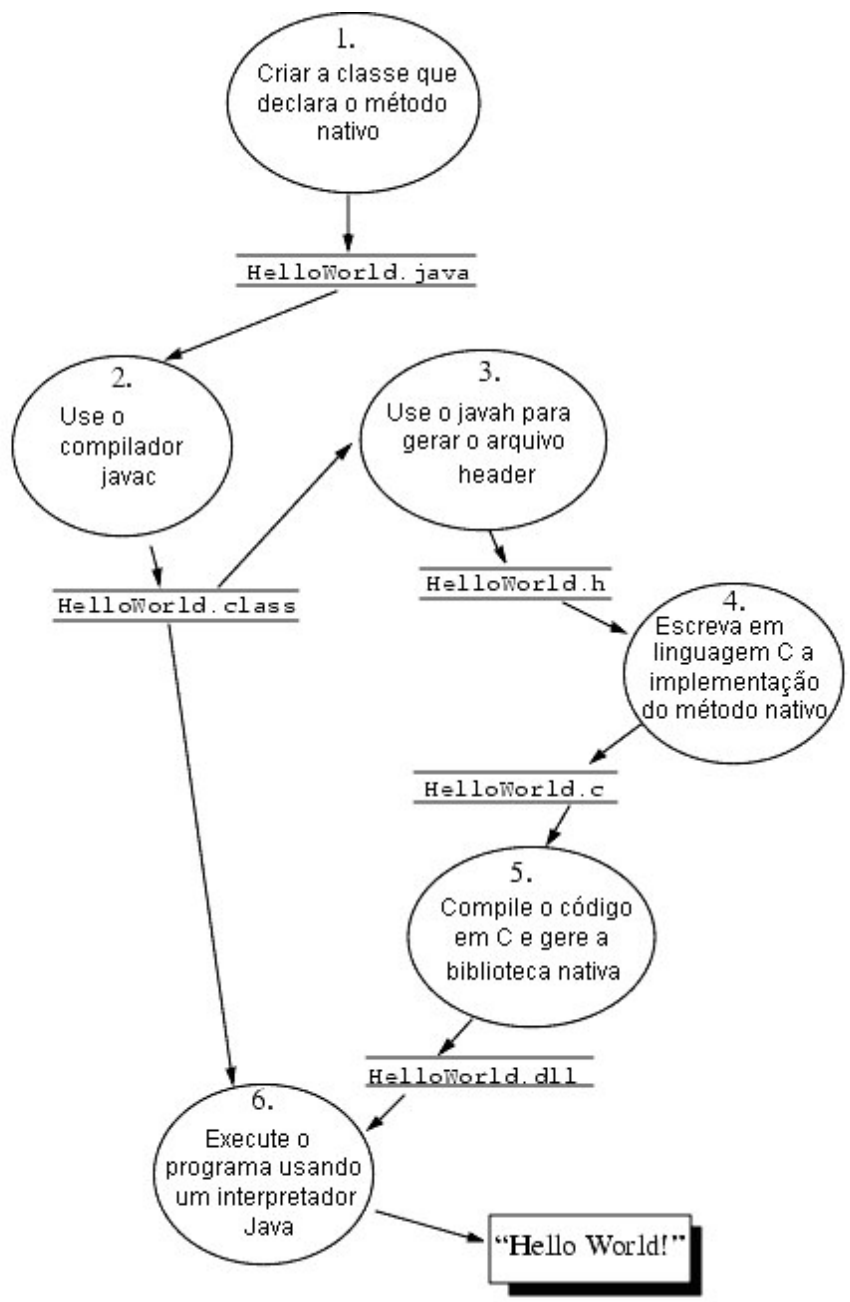

Figura 27. Processo de implementação de uma classe Java com JNI. Baseado em JNI (2007).

A.1 DECLARAR O MÉTODO NATIVO 
A seguir é apresentado um exemplo de programa escrito na linguagem Java, que define a classe chamada HelloWorld a qual contém o método nativo, print.

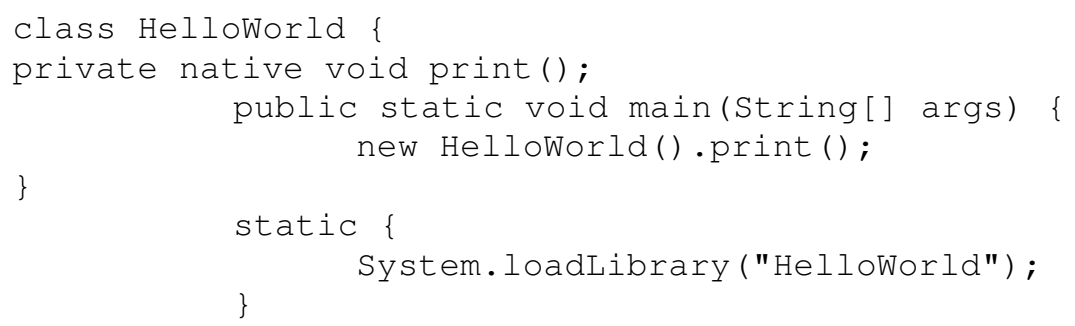

A classe HelloWorld começa com a declaração do método nativo print. Em seguida no método main é instanciada a classe HelloWorld e invocado o método nativo print deste objeto. A última parte da classe é a definição de inicialização estática, que carrega o conteúdo da biblioteca que implementa o método nativo print. No sistema operacional Windows ele será buscado pela biblioteca "HelloWorld.dll"; já nos sistemas UNIX's será procurada a biblioteca "libHelloWorld.so".

\section{A.2 COMPILAR A CLASSE HELLOWORLD}

Depois de definida a classe HelloWorld, deve-se gravar o código-fonte em um arquivo chamado HelloWorld. java. Então deve-se compilar o arquivo usando o compilador javac que vêm com o pacote Java Development Kit (JDK).

javac HelloWorld.java

Este comando irá gerar o arquivo HelloWorld.class no mesmo diretório.

\section{A.3 CRIAR O ARQUIVO HEADER NATIVO}

Em seguida deve-se utilizar a ferramenta javah para gerar o arquivo header no JNI-style que é muito usado quando se implementa métodos nativos em linguagem $\mathrm{C}$. Executar o seguinte comando: 
O nome do arquivo header é o nome da classe com a extensão “.h”. Esse comando irá gerar o arquivo HelloWorld.h. A parte mais importante deste arquivo é o protótipo da função Java_HelloWorld_print, que é a função em linguagem C que implementa o método HelloWorld.print.

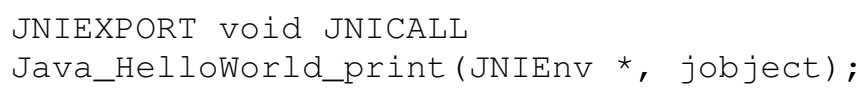

Deve-se observar que existem dois argumentos para a função correspondente. O primeiro argumento, para toda implementação de método nativo, é um ponteiro para um tipo JNIEnv. O segundo argumento é uma referência ao próprio objeto HelloWorld, equivalente ao ponteiro this.

\section{A.4 ESCREVER A IMPLEMENTAÇÃO DO MÉTODO NATIVO}

O arquivo gerado pelo comando javah irá ajudar na escrita da implementação do método nativo nas linguagens $\mathrm{C}$ ou $\mathrm{C}_{++}$, através do protótipo especificado no arquivo gerado. A seguir apresenta-se o arquivo HelloWorld.c com o método HelloWorld.print implementado:

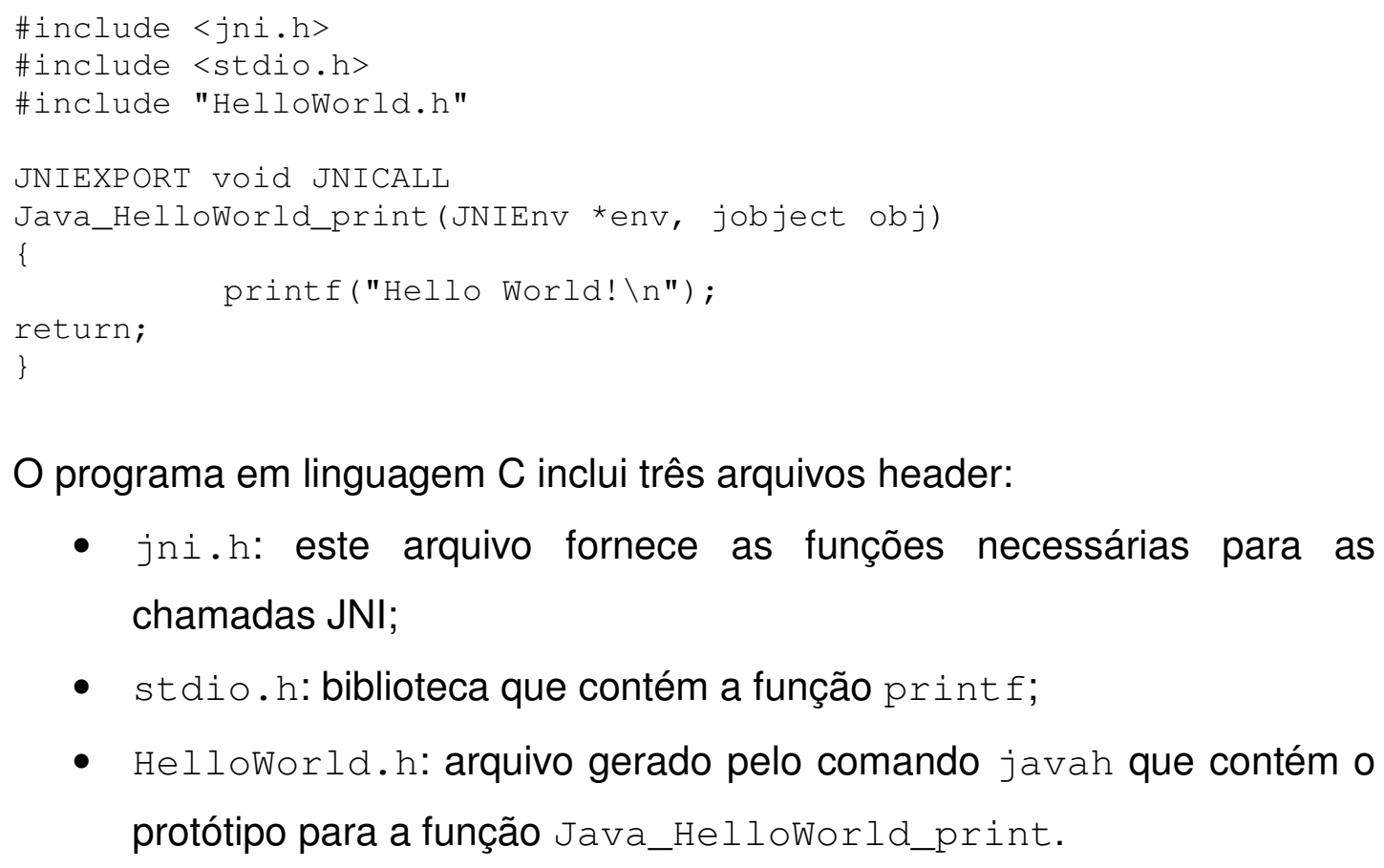

O programa em linguagem $\mathrm{C}$ inclui três arquivos header:

- jni.h: este arquivo fornece as funções necessárias para as chamadas JNI;

- stdio.h: biblioteca que contém a função printf;

- HelloWorld.h: arquivo gerado pelo comando javah que contém o protótipo para a função Java_HelloWorld_print. 


\section{A.5 COMPILAR O CÓDIGO .C E CRIAR UMA BIBLIOTECA NATIVA}

Quando foi criada a classe HellWorld no arquivo HelloWorld.java, você incluiu-se a linha de código para carregar a biblioteca nativa no programa:

System.loadLibrary("HelloWorld”);

Agora que o programa em linguagem $C$ já esta escrito, há a necessidade de compilá-lo e de criar a biblioteca nativa. Existem diferentes maneiras de construir uma biblioteca nativa, que depende do seu sistema operacional e do compilador disponível. Seguem alguns exemplos:

\section{A.5.1 SOLARIS}

Executar o seguinte comando que cria uma biblioteca com o nome libHelloWorld.so:

CC -G -I/java/include -I/java/include/solaris
HelloWorld.c -o libHelloWorld.so

\section{A.5.2 WINDOWS}

Para esse sistema operacional exemplifica-se o comando utilizando-se os compiladores Microsoft Visual $\mathrm{C}++$ e o GCC.

- Microsoft Visua C++:

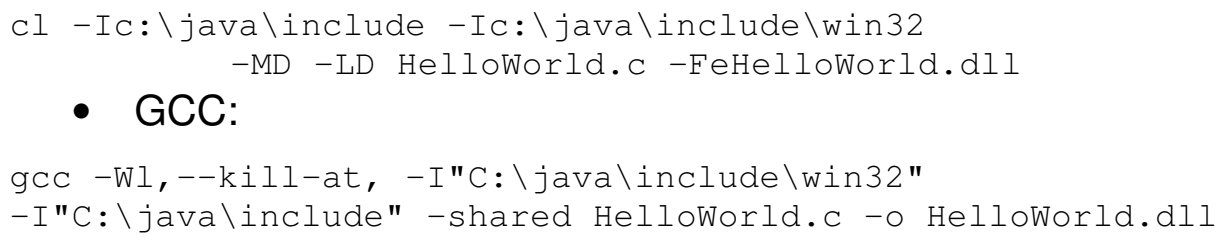

Em ambos os casos a biblioteca criada irá se chamar HelloWorld.dll.

\section{A.5.3 UNIX}

A seguir, apresenta-se o comando para o sistema UNIX usando o compilador GCC. Ele irá gerar uma biblioteca com o nome libHelloWorld.so. 
gcc -o libHelloWorld.so -shared -Wl,-soname, libHelloWorld.so

-I/export/home/jdk1.2/include

-I/export/home/jdk1.2/include/linux

HelloWorld.c -static -lc

\section{A.6 EXECUTAR O PROGRAMA}

Neste ponto, tem-se dois componentes prontos para serem executados: a classe que chama o método nativo e a biblioteca que implementa o método nativo.

Como a classe HelloWorld contém o seu próprio método main, ela pode ser executada da seguinte maneira:

java HelloWorld

A seguinte saída poderá ser visualizada:

\section{Hello World!}

É importante informar corretamente o endereço da biblioteca para o programa. Existe uma lista de diretórios de bibliotecas (library path) em que a máquina virtual do Java procura a biblioteca nativa para carregar. Se o endereço da biblioteca não for informado corretamente e ela não for encontrada, será exibida uma mensagem de erro similar a apresentada abaixo:

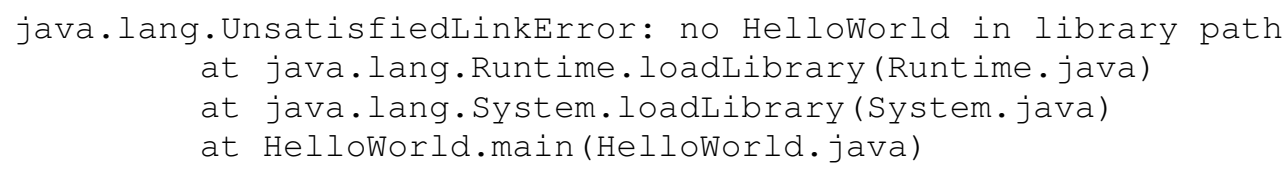

Deve-se ter certeza de que a biblioteca se encontra em um dos diretórios pertencentes ao library path da máquina virtual do Java. O endereço da biblioteca também pode ser especificado pela linha de comando em Java, como é apresentado a seguir:

java -Djava.library.path=. HelloWorld

O parâmetro "-D" informa à máquina virtual do Java para inserir o diretório "." em sua lista de diretórios. 


\section{APÊNDICE B - LON-NODE-MIB}

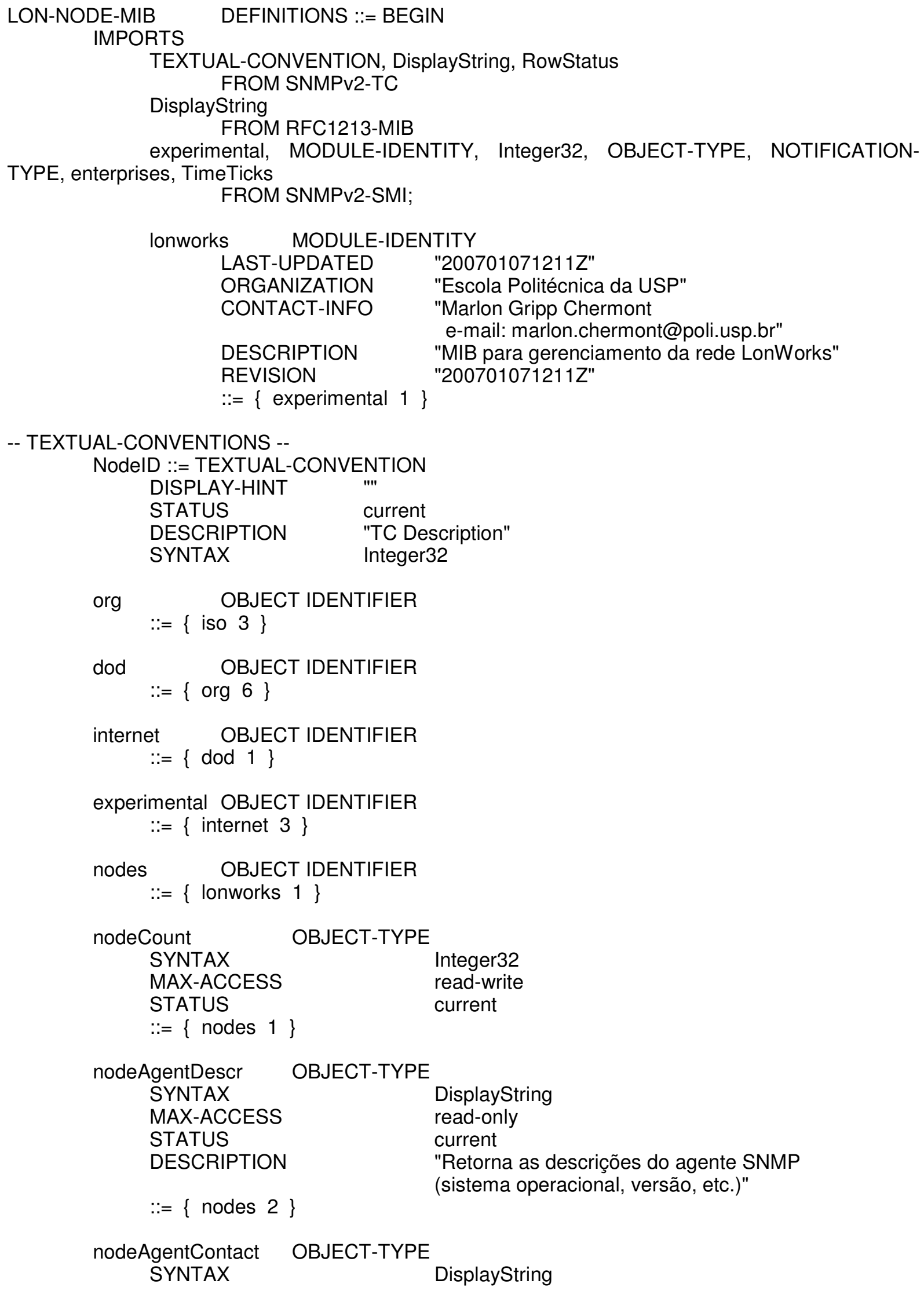




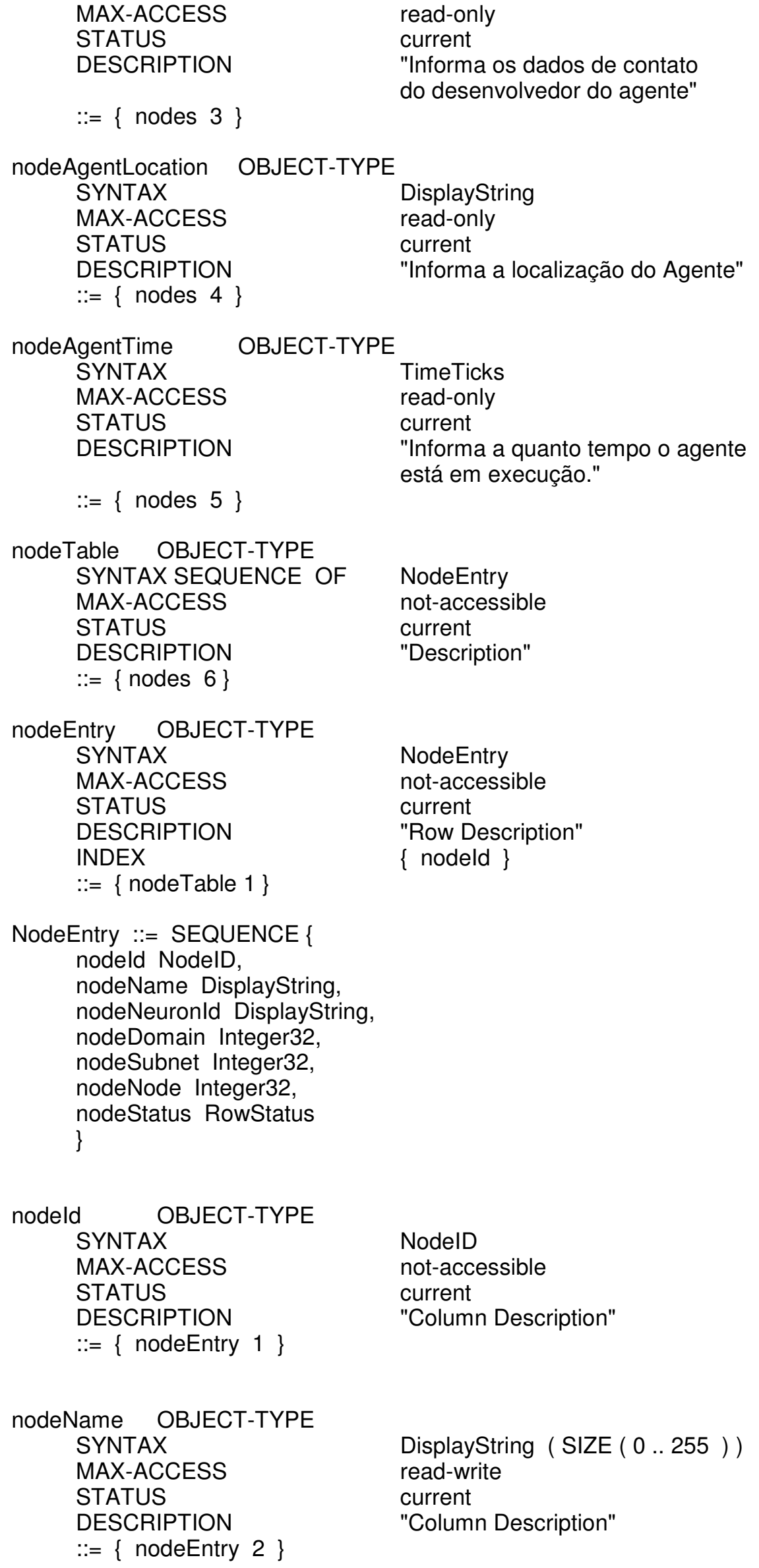




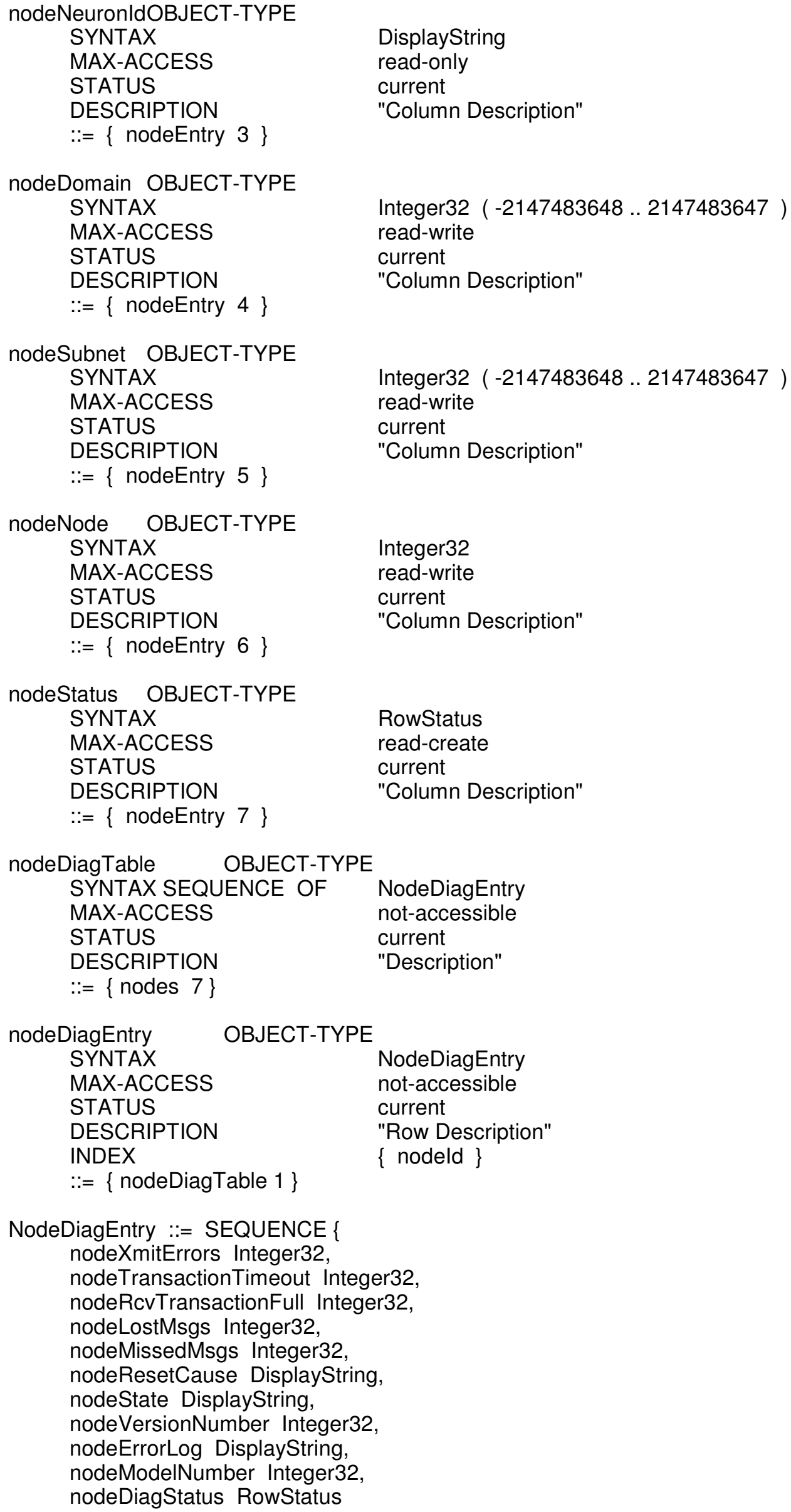




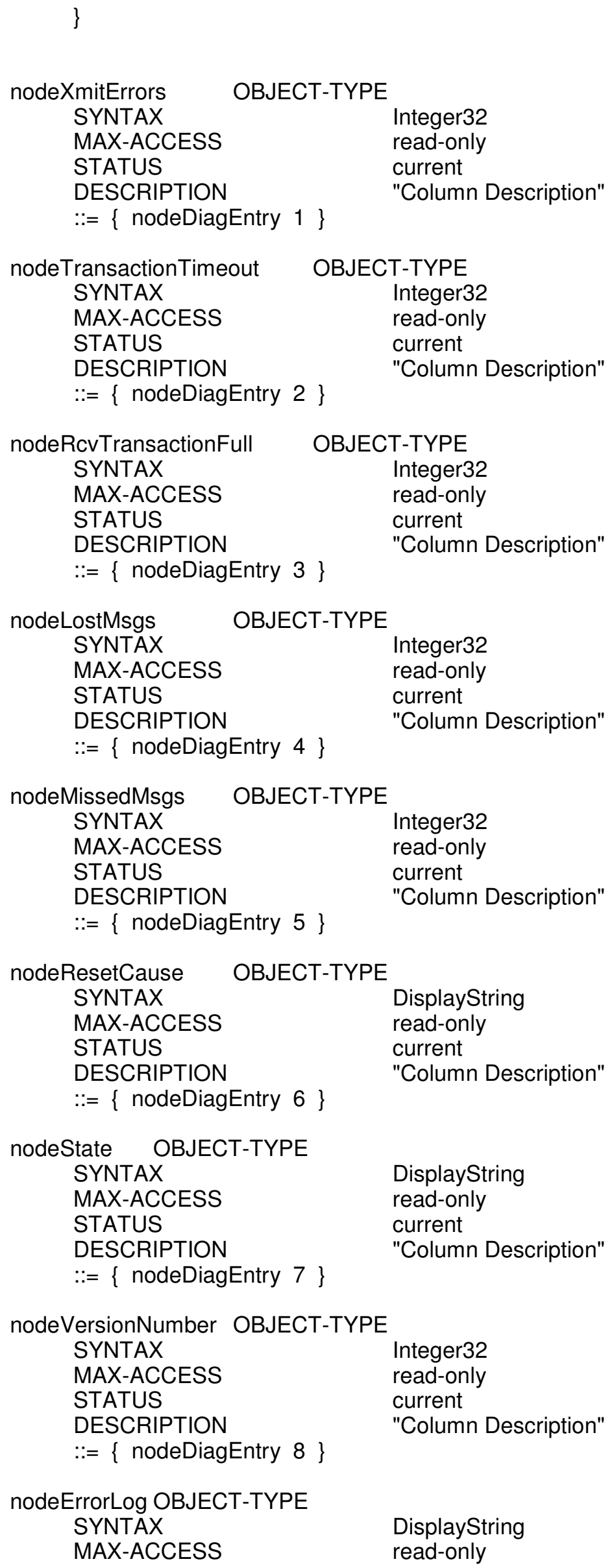




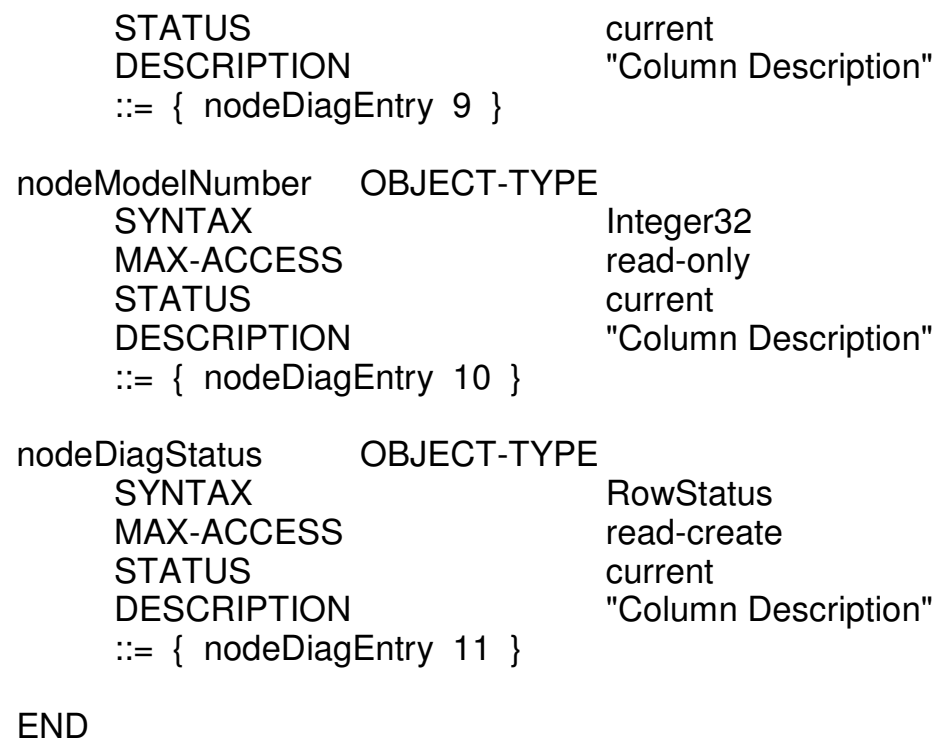

